\title{
DERIVATION OF THE DYNAMICS EQUATIONS FOR RECEIVER AIRCRAFT IN AERIAL REFUELING
}

\author{
by \\ JAYME WAISHEK
}

Presented to the Faculty of the Graduate School of

The University of Texas at Arlington in Partial Fulfillment of the Requirements

for the Degree of

MASTER OF SCIENCE IN AEROSPACE ENGINEERING

THE UNIVERSITY OF TEXAS AT ARLINGTON

December 2007 
Copyright @ by Jayme Waishek 2007

All Rights Reserved 
For my Fida 


\section{ACKNOWLEDGEMENTS}

I would like to thank the University of Texas at Arlington as well as its Mechanical and Aerospace Engineering Department, Honors College, Alumni Association, and many other affiliates, both personal and corporate, which have financially supported me throughout my college career. I would also like to thank the Texas Space Grant Consortium and Bell Helicopter Textron, Inc., for their particular financial support and recognition throughout my graduate studies.

I am highly grateful for my supervising professor, Dr. Atilla Dogan, and the relentless guidance he provided me throughout this and other projects, which enabled me to publish a technical paper and submit a journal article during my MS studies. I greatly appreciated the research assistantship he was able to offer during the first half of our work together. I am further appreciative of the time spent and interest shown by Dr. Donald Wilson and Dr. Kent Lawrence as they reviewed this thesis and participated in my defense committee.

Finally, I would like to acknowledge all of the UTA faculty and teachers from years before, as well as family and friends, who encouraged me, through praise and criticism, to always learn more than the minimum required. Their advice will stay with me through the rest of my endeavors.

November 21, 2007 


\author{
ABSTRACT \\ DERIVATION OF THE DYNAMICS EQUATIONS \\ FOR RECEIVER AIRCRAFT \\ IN AERIAL REFUELING \\ Publication No. \\ Jayme Waishek, M.S. \\ The University of Texas at Arlington, 2007
}

Supervising Professor: Atilla Dogan

This thesis describes the derivation of a new set of nonlinear, 6-DOF equations of motion of a receiver aircraft undergoing an aerial refueling, including the effect of timevarying mass and inertia properties associated with the fuel transfer and the tanker's vortex induced wind effect. Since the center of mass of the receiver is time-varying during the fuel transfer, the equations are written in a reference frame that is geometrically fixed in the aircraft. Due to the fact that aerial refueling simulation and control deal with the position and orientation of the receiver relative to the tanker, the equations of motion are derived in terms of the translational and rotational position and velocity with respect to the tanker. Further, for the derivation, Newton's law is applied to the system, which consists of the receiver aircraft and the fuel before and after being transferred into the receiver. The new receiver equations of motion are implemented in an integrated simulation environment with a feedback controller for receiver station-keeping as well as 
the full set of nonlinear, 6-DOF equations of motion of the tanker aircraft and a feedback controller to fly the tanker on a U-turn maneuver. 


\section{TABLE OF CONTENTS}

ACKNOWLEDGEMENTS .............................. iv

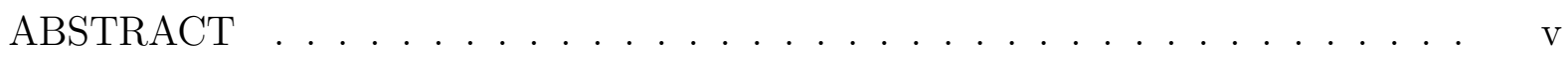

LIST OF FIGURES . . . . . . . . . . . . . . . . . . . ix

Chapter

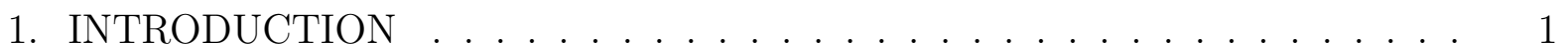

2. MATHEMATICAL CONCEPTS ...................... 4

2.1 Vectrix Formalism . . . . . . . . . . . . . . . . . . . 4

2.2 Tanker's body frame . . . . . . . . . . . . . . . . . . 5

2.3 Receiver's body frame . . . . . . . . . . . . . . . 6

2.4 Rotation matrices . . . . . . . . . . . . . . . . . . . . 6

2.5 Matrix and Vector Properties . . . . . . . . . . . . . . 8

3. TRANSLATIONAL MOTION . . . . . . . . . . . . . . 11

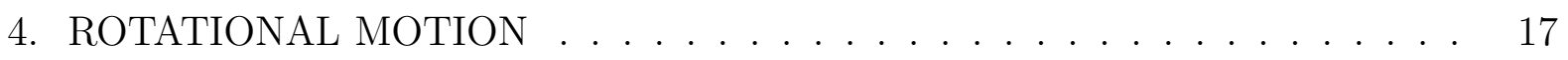

5. DERIVATION OF THE EQUATIONS IN MATRIX FORM . . . . . . . . . . 21

5.1 Translational Kinematics . . . . . . . . . . . . . . . . 21

5.2 Rotational Kinematics . . . . . . . . . . . . . . . 23

5.3 Translational Dynamics . . . . . . . . . . . . . . . . . 24

5.4 Rotational Dynamics . . . . . . . . . . . . . . . . . . 28

6. STATE-SPACE FORM OF THE DYNAMICS EQUATIONS . . . . . . . . 41

7. MODELING THE VORTEX AND ITS EFFECT . . . . . . . . . . . . 43

8. APPLICATION . . . . . . . . . . . . . . . . . . 45

8.1 Fuel Tank Configuration . . . . . . . . . . . . . . . . . . 45 
8.2 Simulation Results . . . . . . . . . . . . . . . . . . . 49

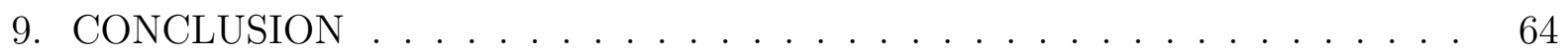
Appendix

A. ROTATIONAL KINEMATICS DERIVATION . . . . . . . . . . . . . . 67

B. DERIVATIONS OF POSITION VECTORS IN MATRIX FORM . . . . . . . 71

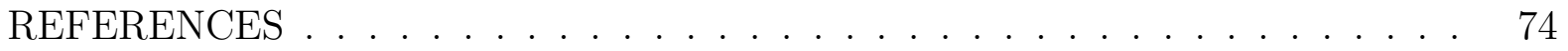

BIOGRAPHICAL STATEMENT . . . . . . . . . . . . . . . . 81 


\section{LIST OF FIGURES}

Figure $\quad$ Page

2.1 Intermediate reference frames: Tanker and Receiver's body frame . . . . 5

2.2 Various reference frames and the rotation matrices between them . . . . 6

3.1 Receiver-Fuel System of Constant Mass . . . . . . . . . . . . . . . . . . . 12

8.1 Receiver aircraft with its fuel tanks . . . . . . . . . . . . . 46

8.2 Initial condition for lumped mass position vector . . . . . . . . . . . 47

8.3 Two-view diagram of fuel tank $1 \ldots \ldots$. . . . . . . . . . . 48

8.4 Receiver fuel tank failure cases . . . . . . . . . . . . . . . . . . . . . 49

8.5 Straight level flight position deviation . . . . . . . . . . . . . . 51

8.6 Receiver deviation in relative orientation in straight level flight . . . . . . 52

8.7 Straight level flight position deviation time history . . . . . . . . . . 53

8.8 Straight level flight orientation deviation time history . . . . . . . . . . 54

8.9 Time history of receiver control surface deflections in straight level flight . 55

8.10 Time history of receiver throttle setting in straight level flight . . . . . 56

8.11 Turning flight position deviation . . . . . . . . . . . . . . . . 57

8.12 Receiver deviation in relative orientation during turn . . . . . . . . 58

8.13 Turning flight position deviation time history . . . . . . . . . . . . 58

8.14 Time history of the receiver relative orientation deviation during turn . . 59

8.15 Time history of receiver control surface deflections during turn . . . . . 60

8.16 Time history of receiver throttle setting during turn . . . . . . . . . 60

8.17 Time history of receiver in straight level flight becoming fully refueled . . 61

8.18 Turning flight position deviation for vortex and refueling comparison . . . 61 
8.19 Turning flight orientation deviation for vortex and refueling comparison . 


\section{CHAPTER 1 \\ INTRODUCTION}

In aerial refueling operations, fuel is transferred from one aircraft (the tanker) to another (the receiver), both of them being airborne. This operation might be needed to sustain/complete some critical flight missions that require an endurance or range beyond what the aircraft, either military or commercial [1], was designed for. The important modeling and control problems studied in the context of aerial refueling $[2,3,4,5,6,7]$ include models of the tanker-receiver interference, stability of the receiver aircraft and design of an autonomous refueling controller. There has been a great interest in the research community [7] to develop an autonomous and efficient control algorithm that would enable a successful aerial refueling, with least disturbance effect on the receiver aircraft during the whole operation. In the design, development and validation of such a control algorithm, a mathematical model and computer simulation of the receiver dynamics during the refueling is essential. In order to generate authentic results from the simulation, it is critical to model the receiver aircraft with sufficient accuracy, taking into account all major factors that influence its dynamics. Specifically, this work derives equations of motion for the receiver to model (i) the relative motion of the receiver aircraft with respect to the tanker aircraft, (ii) time-varying mass and inertia properties during fuel transfer, and (iii) exposure to nonuniform wind induced by the tanker's wake vortex.

In the problem of aircraft formation flight, various methods are used for formulating relative motion. Some papers $[8,9,10,11,12,13]$ consider only relative position. Others $[14,15,16,17,18]$ partially consider orientation in formulating relative position. Further, the problem of relative motion is studied in other applications such as spacecraft 
formation $[19,20,21,22,23]$ and aircraft landing on a ship $[24,25,26]$. A common approach among the work reported above is to quantify relative motion through kinematic relations after the equations of motion are derived for each aircraft separately. In this work, the equations of motion are derived in terms of relative position and orientation. A benefit of this approach is that the motion of the tanker aircraft is explicitly formulated as disturbance on the relative motion of the receiver aircraft [27, 28].

Mass properties of a system will vary when mass transfer into or out of the system occurs or mass distribution within the system changes. Variable mass systems are studied extensively in the area of space flight $[29,30,31,32,33,34,35,36,37]$, which show the significance of the mass transfer effect on vehicle dynamics. The system of rotorcraft with external load [38] and the Concorde supersonic airline with weight distribution changed by moving fuel $[39,40,41]$ are examples of systems in which mass changes location. Other examples are space and underwater vehicles $[42,43,44]$ where shape change actuation or moving internal masses are used for control. In the case of aerial refueling, fuel transferred to the receiver aircraft brings in momentum and changes its inertia properties. Previous works $[45,7,46,47,48,49,50,51,1,52]$ in aerial refueling either ignore the effect of mass transfer or treat it as disturbance causing parametric uncertainty [7]. In this work, the equations of motion for the receiver are derived considering the system of the receiver aircraft and the fuel before and after being transferred into the receiver. This yields a generic mathematical model that includes the dynamic effect of fuel transfer.

The dynamic effect of nonuniform wind is another factor considered in this work. In aerial refueling operation, like any other close formation flight, the follower aircraft (receiver in this case) is subject to the nonuniform wind induced by the wake vortex of the lead aircraft (tanker in this case). The vortex induced wind can be detrimental to the control of the receiver aircraft $[52,53]$. To model the dynamic effect of nonuniform wind, various techniques such as strip theory, averaging, and look-up tables are used $[54,55,56]$. 
Such techniques (i) are limited to a certain operating condition, (ii) are computationally intensive, and (iii) require large aerodynamic databases. Another approach is to avoid modeling of nonuniform wind or treat it as unknown/stochastic disturbance. In this work, the equations of motion are derived such that wind terms are explicitly formulated. This enables the implementation of a novel vortex effect modeling technique $[57,58]$ in the aerial refueling problem. With this technique, the modeling of nonuniform wind effect is more direct and computationally efficient. Further, there is no restriction on the relative position/orientation or sizes of the aircraft.

The remainder of this thesis is organized as follows. Equations for translational kinematics and dynamics are derived in Chapter 3. Similarly, Chapter 4 presents the derivation of the equations for the rotational motion. In Chapter 5 , the vector equations of motion are converted into matrix form. Chapter 7 summarizes Ref. [58], which introduced the procedure for approximating the non-uniform wind field induced by wake vortices as uniform wind field. Chapter 8 explains how the physical design parameters of the receiver aircraft and its fuel tanks are incorporated into the equations of motion, and additionally presents simulation results. Finally, Chapter 9 presents conclusions based on the simulation results and proposes additional application of the concepts herein addressed. 


\section{CHAPTER 2}

\section{MATHEMATICAL CONCEPTS}

\subsection{Vectrix Formalism}

To write a vector in matrix form, vectrix formalism [57] is employed. This facilitates (i) the expression of a vector in terms of its representation in a frame and the unit vectors of the frame and (ii) transforming the representation of a vector to another frame using the corresponding rotation matrix. The vectrix of X-frame is defined to be the array of the unit vectors of its axes and is denoted by

$$
[\underline{\hat{X}}]=\left[\begin{array}{c}
\underline{\hat{\imath}}_{X} \\
\hat{\hat{\jmath}}_{X} \\
\underline{\hat{k}}_{X}
\end{array}\right]
$$

Hence, vector $\underline{a}$ can be written as

$$
\underline{a}=[\underline{\hat{X}}]^{T} a
$$

where $a$ is the representation of vector $\underline{a}$ in X-frame. Note that the relationship between the vectrices of the two frames are defined by the rotation matrix between the two frames, namely

$$
[\underline{\hat{X}}]=\mathbf{R}_{\mathbf{X Y}}[\underline{\hat{Y}}]
$$

where $\mathbf{R}_{\mathbf{X Y}}$ is the rotation matrix from Y-frame to X-frame. Note that all matrices in this thesis are written in bold font. Note also that rotation matrix is used to find the representation of a vector in a frame using the representation of the same vector in 
another frame. For example, let $a_{Y}$ be the representation of vector $\underline{a}$ in $Y$-frame. Then, the representation of vector $\underline{a}$ in $X$-frame is

$$
a=\mathbf{R}_{\mathbf{X Y}} a_{Y}
$$

where $a$ and $a_{Y}$ are $(3 \times 1)$ arrays.

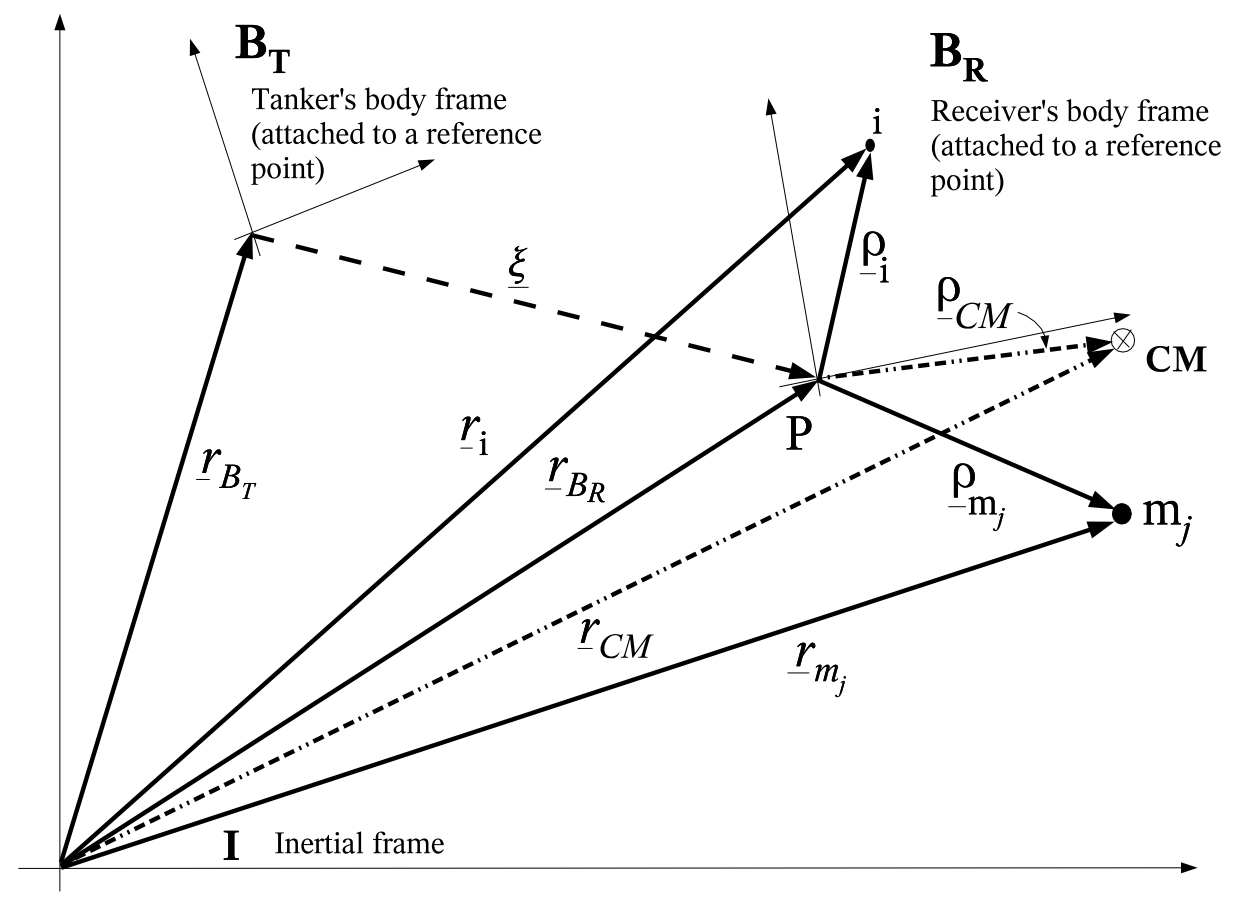

Figure 2.1. Intermediate reference frames: Tanker and Receiver's body frame.

\subsection{Tanker's body frame}

This frame is fixed to the tanker's body at a reference point which could be its center of mass $(\mathrm{CM})$ or any geometric reference point, and moving and rotating with the tanker. From this point on, this frame will be referred to as the "Tanker's Body frame" or " $B_{T}$-frame". Its vectrix is denoted by $\left[\underline{\hat{B}}_{T}\right]$. Since during the refueling, the 
position and orientation of the receiver need to be controlled relative to the tanker, the translational positions and angular orientations are defined with respect to $B_{T}$-frame.

\subsection{Receiver's body frame}

This frame is fixed to the receiver aircraft's body at a geometrically fixed reference point, $P$, and is called the "Receiver's Body frame" whose vectrix is denoted by $\left[\underline{B}_{R}\right]$. For the dynamic model developed in this thesis, the reference point $P$ on the receiver aircraft is considered to be the $\mathrm{CM}$ of the empty receiver and stays fixed at that position even though the CM would move during the refueling. This frame is chosen as the body frame of the receiver to accommodate the fact that the CM will be moving during fuel transfer. This frame is hereafter denoted as $B_{R}$-frame. Fig. 2.1 depicts the spatial relation of three reference frames: (i) Inertial, (ii) Tanker's body ( $B_{T}$-frame), and (iii) Receiver's body $\left(B_{R}\right.$-frame).

\subsection{Rotation matrices}

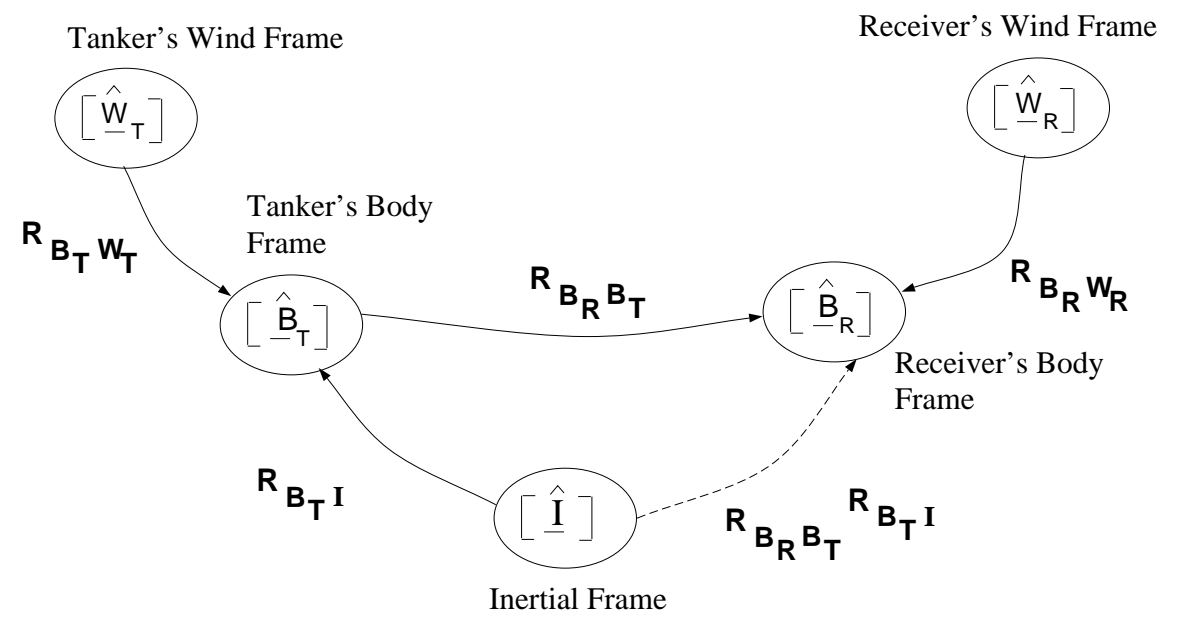

Figure 2.2. Various reference frames and the rotation matrices between them. 
In the development of the equations of motion in this thesis, there are five frames involved: (i) inertial frame, (ii) $B_{T}$-frame, (iii) tanker's wind frame, (iv) $B_{R}$-frame and (v) receiver's wind frame ( $W_{R}$-frame) (Fig. 2.2). Since the focus is on the dynamics of the receiver aircraft relative to the tanker, the nonlinear 6-DOF equations of motion of the receiver are written in $B_{R}$-frame. However, the vectors used in the derivation will be initially written in the respective most convenient frame. For example, the gravitational force vector will be written in the inertial frame while the velocity of the receiver relative to the air will be written in $W_{R}$-frame. To transform all the vectors and their representations written in the most convenient frames to the frame in which the equations of motion are written, the rotation matrices defined between the vectrices of each pair of the frames will be used as shown in Fig. 2.2.

For example, the relationship between the vectrices of the inertial frame and $B_{T^{-}}$ frame is

$$
\left[\underline{\hat{B}}_{T}\right]=\mathbf{R}_{\mathbf{B}_{\mathbf{T}} \mathbf{I}}[\underline{\hat{I}}]
$$

where $\mathbf{R}_{\mathbf{B}_{\mathbf{T}} \mathbf{I}}$ is the rotation matrix from the inertial frame to $B_{T}$-frame. By the orthonormality of rotation matrices, the transformation from $B_{T}$-frame to the inertial frame is obtained simply by taking transpose of $\mathbf{R}_{\mathbf{B}_{\mathrm{T}} \mathbf{I}}$. Thus,

$$
[\underline{\hat{I}}]=\mathbf{R}_{\mathbf{B}_{\mathbf{T}} \mathbf{T}}^{\mathbf{T}}\left[\underline{\hat{B}}_{T}\right]
$$

When the rotation matrix from the inertial frame to the receiver's body frame is needed, as Fig. 2.2 implies, in this thesis, it is written through $B_{T}$-frame, i.e.

$$
\mathbf{R}_{\mathrm{B}_{\mathrm{R}} \mathrm{I}}=\mathbf{R}_{\mathrm{B}_{\mathrm{R}} \mathrm{B}_{\mathrm{T}}} \mathbf{R}_{\mathrm{B}_{\mathrm{T}} \mathbf{I}}
$$

which means a transformation from the inertial frame to $B_{T}$-frame and then from there to $B_{R}$-frame. The reason for not using the rotation matrix from the inertial frame directly 
to $B_{R}$-frame is because, in aerial refueling, the focus is on the motion of the receiver relative to the tanker. Thus,

$$
\left[\underline{\hat{B}}_{R}\right]=\mathbf{R}_{\mathbf{B}_{\mathbf{R}} \mathbf{B}_{\mathbf{T}}} \mathbf{R}_{\mathbf{B}_{\mathbf{T}} \mathbf{I}}[\underline{\hat{I}}]
$$

or

$$
[\underline{\hat{I}}]=\mathbf{R}_{\mathbf{B}_{\mathbf{T}} \mathbf{I}}^{\mathbf{T}} \mathbf{R}_{\mathbf{B}_{\mathbf{R}} \mathbf{B}_{\mathbf{T}}}^{\mathbf{T}}\left[\underline{\hat{B}}_{R}\right]
$$

Similarly, the relation between the vectrices of $W_{R}$-frame and $B_{R}$-frame is

$$
\left[\underline{\hat{B}}_{R}\right]=\mathbf{R}_{\mathbf{B}_{\mathbf{R}} \mathbf{w}_{\mathbf{R}}}\left[\underline{\hat{W}}_{R}\right]
$$

where $\mathbf{R}_{\mathbf{B}_{\mathbf{R}} \mathbf{W}_{\mathbf{R}}}$ is the rotation matrix from $W_{R^{-}}$frame to $B_{R}$-frame.

\subsection{Matrix and Vector Properties}

This section presents for easy referencing the matrix and vector properties used in the derivation of the equations of motion. Further, proofs are provided for some of the facts presented. For the expression and proof of the facts, the following notation is used.

There are two reference frames with vectrices $[\underline{\hat{1}}]$ and $[\underline{\hat{2}}]$. The rotation matrix from the first frame to the second is defined to be $\mathbf{R}_{\mathbf{2 1}}$. There are three vectors, $\underline{a}$, $\underline{b}$, and $\underline{c}$, with corresonding representations $a, b$, and $c$. The representations are 3 -by-1 column matrices constructed by the components of the vector in the corresponding frame. Vector products are denoted by $\times$ while $\cdot$ refers to scalar product of vectors. Superscript $T$ denotes the transpose of a matrix.

Fact 1: The triple product identity

$$
\underline{a} \times(\underline{b} \times \underline{c})=(\underline{a} \cdot \underline{c}) \underline{b}-(\underline{a} \cdot \underline{b}) \underline{c}, \quad \forall \underline{a}, \underline{b}, \underline{c}
$$

Fact 2: Scalar product of vectors through their representations

$$
\underline{a} \cdot \underline{b}=a^{T} b, \quad \forall \underline{a}, \underline{b},
$$


Fact 3: Relation between representation of vectors

$$
a^{T} b c=c a^{T} b, \quad \forall \underline{a}, \underline{b}, \underline{c},
$$

Fact 4: Quadruple product of vectors

$$
\underline{a} \times[\underline{b} \times(\underline{c} \times \underline{d})]=(\underline{b} \cdot \underline{d}) \underline{a} \times \underline{c}-(\underline{b} \cdot \underline{c}) \underline{a} \times \underline{d}, \quad \forall \underline{a}, \underline{b}, \underline{c}, \underline{d}
$$

Proof: By extension of Eq. (2.11).

Fact 5: Vector product of vectors through their representations

$$
\begin{aligned}
\underline{a} \times \underline{b} & =[\underline{\hat{1}}]^{T} a \times[\underline{\hat{2}}]^{T} b \\
& =-[\underline{\hat{1}}]^{T} \mathbf{S}(a) b
\end{aligned}
$$

where $a$ and $b$ are representations of $\underline{a}$ and $\underline{b}$, respectively, in the frame having vectrix $[\hat{1}]$. Note that $\mathbf{S}(a)$ is a skew-symmetric matrix constructed with representation $a$ such that

$$
\mathbf{S}(a)=\left[\begin{array}{ccc}
0 & a_{3} & -a_{2} \\
-a_{3} & 0 & a_{1} \\
a_{2} & -a_{1} & 0
\end{array}\right]
$$

for vector $\underline{a}$ with representation $\left[\begin{array}{lll}a_{1} & a_{2} & a_{3}\end{array}\right]^{T}$ in the frame having vectrix $[\hat{1}]$.

Fact 6: Order of vectors in vector product

$$
\mathbf{S}(a) b=-\mathbf{S}(b) a
$$

Proof: This follows from Eq. (2.15) and the fact that $\underline{a} \times \underline{b}=-\underline{b} \times \underline{a}$.

Fact 7:

$$
\left(a^{T} b\right) \mathbf{S}(a)=-\mathbf{S}(b) a a^{T} \quad \forall a, b
$$

Proof: Note from Eqs. (2.12) and (2.15) that

$$
(\underline{a} \cdot \underline{b}) \underline{a} \times \underline{b}=-[\underline{\hat{1}}]^{T}\left(a^{T} b\right) \mathbf{S}(a) b
$$


Further note that changing the order of a vector product results in the negative of the original product. This can be expressed as

$$
\begin{aligned}
(\underline{a} \cdot \underline{b}) \underline{a} \times \underline{b} & =-(\underline{a} \cdot \underline{b}) \underline{b} \times \underline{a} \\
& =[\underline{\hat{1}}]^{T}\left(a^{T} b\right) \mathbf{S}(b) a
\end{aligned}
$$

by again using Eqs. (2.12) and (2.15). Because $a^{T} b$ is a scalar, Eq. (2.20) can be rearranged to be

$$
(\underline{a} \cdot \underline{b}) \underline{a} \times \underline{b}=[\underline{\hat{1}}]^{T} \mathbf{S}(b) a a^{T} b
$$

The comparison of Eqs. (2.19) and (2.21) leads to Eq. (2.18).

Fact 8:

$$
\mathbf{S}\left(\mathbf{R}_{21} a\right)=\mathbf{R}_{21} \mathbf{S}(a) \mathbf{R}_{21}^{\mathbf{T}}
$$

Proof: As stated previously, let $a$ and $b$ be the representations of $\underline{a}$ and $\underline{b}$, respectively, in the frame having vectrix $[\underline{\hat{1}}]$, namely $\underline{a}=[\underline{\hat{1}}]^{T} a$ and $\underline{b}=[\underline{\hat{1}}]^{T} b$. Note that $[\underline{\hat{2}}]=\mathbf{R}_{\mathbf{2 1}}[\underline{\hat{1}}]$ since $\mathbf{R}_{\mathbf{2 1}}$ is the rotation matrix from the frame with vectrix $[\underline{\hat{1}}]$ to that with $[\underline{\hat{2}}]$. Thus, vectors $\underline{a}$ and $\underline{b}$ can be expressed in terms of $[\underline{\hat{2}}]$ as

$$
\begin{aligned}
\underline{a} & =[\underline{\hat{2}}]^{T} \mathbf{R}_{\mathbf{2 1}} a \\
\underline{b} & =[\underline{\hat{2}}]^{T} \mathbf{R}_{\mathbf{2 1}} b
\end{aligned}
$$

The vector product of vectors $\underline{a}$ and $\underline{b}$ using Eq. (2.15) yields

$$
\underline{a} \times \underline{b}=-[\underline{\hat{1}}]^{T} \mathbf{S}(\mathbf{a}) b
$$

The same vector product performed on $\underline{a}$ and $\underline{b}$ as written in Eq. (2.23) results in

$$
\underline{a} \times \underline{b}=-[\underline{\hat{2}}]^{T} \mathbf{S}\left(\mathbf{R}_{21} \mathbf{a}\right) \mathbf{R}_{21} b
$$

Note further that $[\underline{\hat{2}}]^{T}=[\underline{\hat{1}}]^{T} \mathbf{R}_{\mathbf{2 1}}^{\mathbf{T}}$. Thus, Eq. (2.25) becomes

$$
\underline{a} \times \underline{b}=-[\underline{\hat{1}}]^{T} \mathbf{R}_{\mathbf{2 1}}^{\mathbf{T}} \mathbf{S}\left(\mathbf{R}_{21} \mathbf{a}\right) \mathbf{R}_{21} b
$$

The comparison of Eqs. (2.24) and (2.26) leads to Eq. (2.22). 


\section{CHAPTER 3}

\section{TRANSLATIONAL MOTION}

Since, in aerial refueling, the position of the receiver should be controlled relative to the tanker and not relative to the ground, the translational kinematics should be written in terms of the position vector of the receiver with respect to the tanker. The vectorial relation of the origins of the reference frames ( Fig. 2.1) yields

$$
\underline{r}_{B_{R}}=\underline{r}_{B_{T}}+\underline{\xi}
$$

Further, the effect of the wind is incorporated into the kinematics as

$$
\underline{\underline{r}}_{B_{R}}=\underline{U}+\underline{W}
$$

where $\underline{U}$ is the velocity vector of the receiver relative to the surrounding air and $\underline{W}$ is the wind velocity vector, i.e. the velocity of the air relative to the inertial frame. The time derivative of Eq. (3.1) along with Eq. (3.2) yields

$$
\underline{\dot{\xi}}=\underline{U}+\underline{W}-\underline{\dot{r}}_{B_{T}}
$$

which is the translational kinematic equation in terms of the position of the receiver relative to the tanker.

The next step is to derive the translational dynamic equations. The derivation is intended to yield equations with explicit terms to represent dynamics effect of fuel transfer and wind exposure. Due to fuel transfer in refueling, the receiver aircraft is a system of varying mass. Thus, Newton's Second Law, which states that force is equal to the time rate of change of momentum, is not directly applicable. However, it can be applied to a system of varying mass by examining the same mass for the change in momentum at two instances of time [33]. 
This approach is used in this thesis by defining a system of constant mass consisting of the receiver aircraft and the fuel that joins the receiver through a refueling receptacle in a given time interval. Then, the momenta of this system of constant mass before and after fuel joins the receiver are formulated to determine the time rate of change of momentum while refueling.

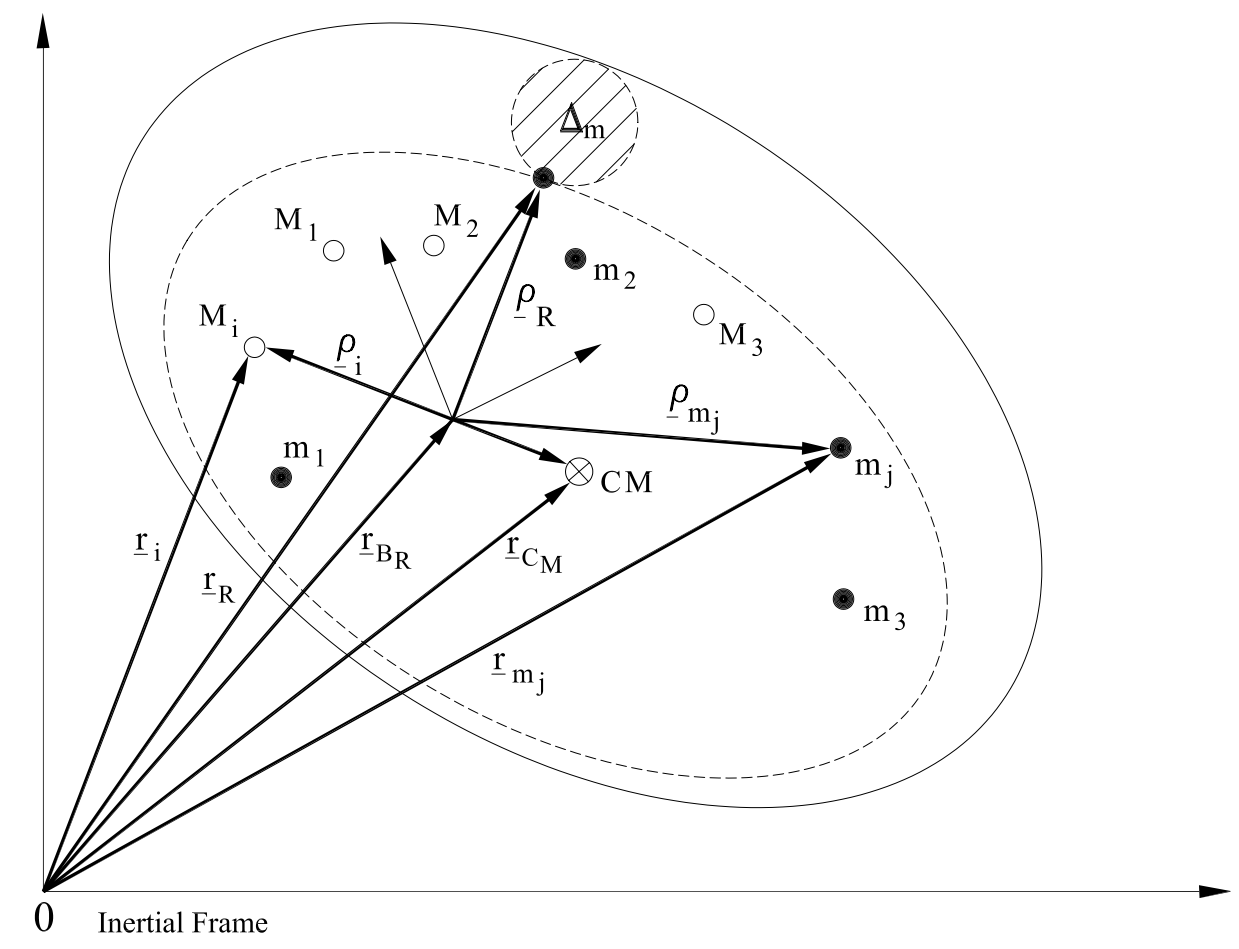

Figure 3.1. Receiver-Fuel System of Constant Mass.

To facilitate the derivation of the dynamics equations including the effect of fuel transfer, the receiver aircraft is considered to comprise two parts (Fig. 3.1). The first part is represented by ' $n$ ' points at fixed positions, $\rho_{i}$, in $B_{R}$-frame with constant masses $M_{i}(i=1, \ldots, n)$. With the rigid body assumption, masses $M_{i}$ are defined to consitute the body of the receiver aircraft excluding the fuel. Additional ' $k$ ' points represent the second part of the receiver. These points are different from the first group in that they 


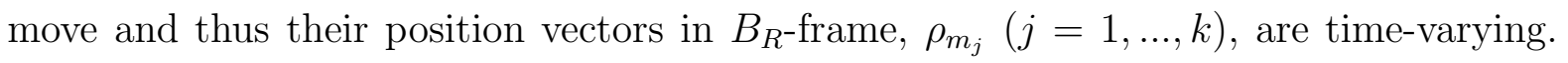
Further, these points represent the various locations of fuel mass after being transferred into the receiver aircraft. The amount of fuel mass, $m_{j}$, assumed to be concentrated at each point, $\rho_{m_{j}}$, might be time-varying during refueling. With the concentrated mass assumption, position $\rho_{m_{j}}$ represents the center of total fuel mass in each fuel tank.

Consider the receiver-fuel system of constant mass depicted in Fig. 3.1, in the time interval $t-\Delta t$ to $t$. Assume that, in this time interval, the fuel $\Delta m$ entering the receiver through the receptacle at $\underline{\rho}_{R}$ splits into ' $k$ ' portions, $\Delta m_{j}$, and each $\Delta m_{j}$ joins $m_{j}$ by the time $t$. The linear momentum of the system at time $t-\Delta t$ is

$$
\underline{P}_{1}=\sum_{i=1}^{n} M_{i} \underline{\dot{r}}_{i}+\sum_{j=1}^{k} m_{j} \underline{\dot{r}}_{m_{j}}+\Delta m \underline{V}_{0}
$$

where $\underline{V}_{0}$ is the velocity of $\Delta m$ relative to the inertial frame, and $\underline{r}_{i}$ and $\underline{r}_{m_{j}}$ are the position vectors of $M_{i}$ and $m_{j}$, respectively (Fig. 3.1). At time $t$, the linear momentum of the system is

$$
\underline{P}_{2}=\sum_{i=1}^{n} M_{i}\left(\underline{\dot{r}}_{i}+\Delta \underline{\dot{r}}_{i}\right)+\sum_{j=1}^{k}\left(m_{j}+\Delta m_{j}\right)\left(\underline{\dot{r}}_{m_{j}}+\Delta \underline{\dot{r}}_{m_{j}}\right)
$$

The difference between the total momenta before and after $\Delta m$ joins the receiver aircraft is found by subtracting Eq. (3.4) from Eq. (3.5) and is calculated to be

$$
\Delta \underline{P}=\sum_{i=1}^{n} M_{i} \Delta \underline{\dot{r}}_{i}+\sum_{j=1}^{k} m_{j} \Delta \underline{\dot{r}}_{m_{j}}+\sum_{j=1}^{k} \Delta m_{j} \underline{\dot{r}}_{m_{j}}+\sum_{j=1}^{k} \Delta m_{j} \Delta \underline{\dot{r}}_{m_{j}}-\Delta m \underline{V}_{0}
$$


The time rate of change of the linear momentum at time $t$ is calculated by dividing Eq. (3.6) by $\Delta t$ and taking the limit as $\Delta t$ approaches 0 . Specifically,

$$
\begin{aligned}
\lim _{\Delta t \rightarrow 0} \frac{\Delta \underline{P}}{\Delta t} & =\underline{\underline{P}} \\
\lim _{\Delta t \rightarrow 0} M_{i} \frac{\Delta \underline{\dot{r}}_{i}}{\Delta t} & =M_{i} \underline{\underline{x}}_{i} \\
\lim _{\Delta t \rightarrow 0} \frac{m_{j} \Delta \underline{\underline{r}}_{m_{j}}}{\Delta t} & =m_{j} \underline{\underline{x}}_{m_{j}} \\
\lim _{\Delta t \rightarrow 0} \frac{\Delta m_{j}}{\Delta t} \underline{\dot{r}}_{m_{j}} & =\dot{m}_{j} \underline{\underline{r}}_{m_{j}} \\
\lim _{\Delta t \rightarrow 0} \frac{\Delta m_{j} \Delta \underline{\dot{r}}_{m_{j}}}{\Delta t} & =0
\end{aligned}
$$

Using these relations, Eq. (3.6) becomes

$$
\underline{\dot{P}}=\sum_{i=1}^{n} M_{i} \underline{\ddot{x}}_{i}+\sum_{j=1}^{k} m_{j} \ddot{\underline{r}}_{m_{j}}+\sum_{j=1}^{k} \dot{m}_{j} \dot{\underline{r}}_{m_{j}}-\dot{m} \underline{V}_{0}
$$

By Newton's Second Law, this is equal to the total force acting on the system at time $t$. This can be rewritten using the facts that $M \underline{\underline{r}}_{B_{R}}$ replaces $\sum_{i=1}^{n} M_{i} \ddot{\underline{x}}_{i}$ because $\sum_{i=1}^{n} M_{i}=$ $M$ and the origin of $B_{R}$-frame is at the $\mathrm{CM}$ of the receiver aircraft before the fuel transfer. Therefore, the total external force vector acting on the receiver aircraft is determined to be

$$
\underline{F}=M \ddot{\underline{r}}_{B_{R}}+\sum_{j=1}^{k}\left(\dot{m}_{j} \dot{\underline{r}}_{m_{j}}+m_{j} \ddot{\underline{\underline{r}}}_{m_{j}}\right)-\dot{m} \underline{V}_{0}
$$

where $\underline{F}$ is the vectorial sum of all external forces.

Equation (3.9) is the translational dynamic equation of the receiver. However, there are three main drawbacks with this form of the equation. First, masses $m_{j}(j=1, \ldots, k)$ will be used to represent the fuel transfer in the fuel tanks located in the receiver aircraft. In that case, the velocity and acceleration of the $m_{j}$ masses will be easier to formulate in $B_{R}$-frame than in the inertial frame. Thus, $\underline{r}_{m_{j}}$ in the second and third terms should 
be written in terms of the position vectors with respect to $B_{R}$-frame. From Fig. 3.1, it is seen that

$$
\underline{r}_{m_{j}}=\underline{r}_{B_{R}}+\underline{\rho}_{m_{j}}
$$

Second, it is easier to express the fuel flow velocity relative to the tanker. It is therefore necessary to separate $\underline{V}_{0}$ into the components associated with the velocity of the tanker, itself, and the velocity of the fuel with respect to the tanker. Thus, the fuel flow velocity $\left(\underline{V}_{0}\right)$ is written as

$$
\underline{V}_{0}=\underline{\dot{r}}_{B_{T}}+\underline{V}_{\dot{m}}
$$

where $\underline{\dot{r}}_{B_{T}}$ is the velocity of the tanker relative to the inertial frame and $\underline{V}_{\dot{m}}$ is the velocity of the fuel relative to the tanker. Substituting Eqs. (3.10) and (3.11) into Eq. (3.9) and rearranging yields

$$
(M+m) \underline{\underline{\underline{r}}}_{B_{R}}=\underline{F}-\dot{m}\left(\underline{\underline{\dot{r}}}_{B_{R}}-\underline{\dot{\underline{r}}}_{B_{T}}+\underline{V}_{\dot{m}}\right)-\sum_{j=1}^{k}\left(\dot{m}_{j} \underline{\dot{\rho}}_{m_{j}}+m_{j} \underline{\ddot{\rho}}_{m_{j}}\right)
$$

where $\sum_{j=1}^{k} m_{j} \underline{\underline{r}}_{B_{R}}$ has been replaced with $m \underline{\underline{\underline{r}}}_{B_{R}}$ and $\sum_{j=1}^{k} \dot{m}_{j} \underline{\dot{q}}_{B_{R}}$ has been replaced by $\dot{m} \underline{\underline{r}}_{B_{R}}$ because $\ddot{\underline{\underline{r}}}_{B_{R}}$ and $\underline{\dot{\underline{r}}}_{B_{R}}$ are independent of $j$, and $\sum_{j=1}^{k} m_{j}=m$.

As a special case, $\dot{m}=\dot{m}_{j}=0$ when the fuel transfer is completed. Then, Eq. (3.12) becomes

$$
(M+m) \underline{\ddot{r}}_{B_{R}}=\underline{F}-\sum_{j=1}^{k} m_{j} \underline{\ddot{\rho}}_{m_{j}}
$$

where the second term is due to the fact that the $\mathrm{CM}$ of the receiver is no longer at point $P$, the origin of $B_{R}$-frame. Further, when the fuel tanks are positioned in such a way that the CM of the fuel is at point $P, \sum_{j=1}^{k} m_{j} \underline{\rho}_{m_{j}}=0$, which implies that $\sum_{j=1}^{k} m_{j} \ddot{\ddot{\rho}}_{m_{j}}=0$ since $m_{j}(j=1, \ldots, k)$ are constant in this special case. Thus, the translational dynamics as represented in Eq. (3.12) is reduced to

$$
(M+m) \ddot{\ddot{r}}_{B_{R}}=\underline{F}
$$


which is as expected because point $P$ is now at the $\mathrm{CM}$ of the system of constant mass, $(M+m)$.

Third, the effect of the wind will be formulated by writing $\underline{\dot{r}}_{B_{R}}$ and $\ddot{\underline{r}}_{B_{R}}$ in terms of the airspeed of the receiver and the wind acting on it. In order to do this, Eq. (3.2) is differentiated to get

$$
\underline{\ddot{r}}_{B_{R}}=\underline{\dot{U}}+\underline{\dot{W}}
$$

Equations (3.2) and (3.15) are substituted into Eq. (3.12) and the result is rearranged to have $\underline{\dot{U}}$ on the left hand side. This results in the general translational dynamics equation:

$$
\underline{\dot{U}}=\frac{1}{(M+m)}\left[\underline{F}-\dot{m}\left(\underline{U}+\underline{W}-\underline{\dot{r}}_{B_{T}}-\underline{V}_{\dot{m}}\right)-\sum_{j=1}^{k}\left(\dot{m}_{j} \underline{\dot{\rho}}_{m_{j}}+m_{j} \underline{\ddot{\rho}}_{m_{j}}\right)\right]-\underline{\dot{W}}
$$

The resultant force acting on the receiver in Eq. (3.16), $\underline{F}$, is considered to be the sum of the gravity force vector $\underline{M}_{R}$, the aerodynamic force vector $\underline{A}_{R}$ and the propulsive force vector $\underline{P}_{R}$.

There are several significant remarks based on the derivation of Eq. (3.16). First, note that the terms containing $\dot{m}_{j}$ and $\dot{m}$ represent the effect of time-varying mass on the translational dynamics of the receiver aircraft. For the aerial refueling scenario, as an example, $\dot{m}_{j}$, the rate of mass change can be approximated as equal to the fuel transfer rate into the $j$ th fuel tank. This modular formulation facilitates the simulation of asymmetric fuel loading conditions. Furthermore, both $\dot{m}$ and $\dot{m}_{j}$ can be time-varying functions, thereby enabling the simulation of the effects of any combination of unsteady and asymmetric fuel transfer. In practice, the values of $\dot{m}_{j}, \underline{\rho}_{m_{j}}, \underline{\dot{\rho}}_{m_{j}}$ and $\underline{\ddot{\rho}}_{m_{j}}$ depend on the shape, size and location of the fuel tanks on the receiver aircraft and also the individual rates of fuel flow into each fuel tank on the receiver. 


\section{CHAPTER 4}

\section{ROTATIONAL MOTION}

For the derivation of the rotational dynamics, the definitions of the receiver-fuel system of constant mass and the reference frames, introduced for translational dynamics, are used. Then, it can be stated that the external moment is equal to the time rate of change of momentum since the momentum of a definite mass is examined for its rate of change [33].

In a manner which parallels the translational dynamics derivation, the angular momenta of the system right before and after fuel $\Delta m$ joins the receiver are used to formulate the time rate of change of the angular momentum. At time $t-\Delta t$, the angular momentum of the system around the origin of the inertial frame is

$$
\underline{H}_{1}=\sum_{i=1}^{n} \underline{r}_{i} \times M_{i} \underline{\underline{r}}_{i}+\sum_{j=1}^{k} \underline{r}_{m_{j}} \times \underline{\dot{r}}_{m_{j}}+\underline{r}_{R} \times \Delta m \underline{V}_{0}
$$

where each term represents the moment of the corresponding linear momentum term in Eq. (3.4) about the origin of the inertial frame. Note that Eq. (4.1) introduces the vector $\underline{r}_{R}$, which is the position of the receiver fuel port relative to the inertial frame. At time $t$ after $\Delta m$ joins the receiver, the angular momentum of the system is determined to be

$$
\underline{H}_{2}=\sum_{i=1}^{n}\left(\underline{r}_{i}+\Delta \underline{r}_{i}\right) \times M_{i}\left(\underline{\dot{r}}_{i}+\Delta \underline{\dot{r}}_{i}\right)+\sum_{j=1}^{k}\left(\underline{r}_{m_{j}}+\Delta \underline{r}_{m_{j}}\right) \times\left(m_{j}+\Delta m_{j}\right)\left(\underline{\dot{r}}_{m_{j}}+\Delta \underline{\dot{r}}_{m_{j}}\right)
$$

where each term represents the moment of the corresponding linear momentum term of Eq. (3.5) about the inertial frame. All the vectors in Eqs. (4.1) and (4.2) are written relative to the inertial frame and the time derivatives are also taken with respect to the inertial frame. The difference between the total angular momenta before and after $\Delta m$ 
joins the receiver aircraft is found by subtracting Eq. (4.1) from Eq. (4.2). This is then calculated to be

$$
\begin{aligned}
\Delta \underline{H} & =\sum_{i=1}^{n} \underline{r}_{i} \times M_{i} \Delta \underline{\dot{r}}_{i}+\sum_{i=1}^{n} \Delta \underline{r}_{i} \times M_{i} \underline{\dot{r}}_{i}+\sum_{j=1}^{k} \underline{r}_{m_{j}} \times \Delta m_{j} \underline{\underline{r}}_{m_{j}} \\
& +\sum_{j=1}^{k} \underline{r}_{m_{j}} \times m_{j} \Delta \underline{\dot{r}}_{m_{j}}+\sum_{j=1}^{k} \Delta \underline{r}_{m_{j}} \times m_{j} \underline{\dot{r}}_{m_{j}}-\underline{r}_{R} \times \Delta m \underline{V}_{0}
\end{aligned}
$$

where the higher-order terms are ignored since they will become zero during the following step. The total external moment vector acting on the receiver aircraft about the origin of the inertial frame is equal to the time rate of change of the angular momentum, which is calculated by dividing Eq. (4.3) by $\Delta t$ and taking the limit as $\Delta t$ approaches 0 using the relations presented in Eq. (3.7). This results in an expression for the total external moment about the origin of the inertial frame at time $t$ as

$$
\underline{M}_{0}=\sum_{i=1}^{n} \underline{r}_{i} \times M_{i} \ddot{\underline{x}}_{i}+\sum_{j=1}^{k} \underline{r}_{m_{j}} \times \dot{m}_{j} \underline{\underline{r}}_{m_{j}}+\sum_{j=1}^{k} \underline{r}_{m_{j}} \times m_{j} \underline{\underline{r}}_{m_{j}}-\underline{r}_{R} \times \dot{m} \underline{V}_{0}
$$

Further note that

$$
\underline{M}_{B_{R}}=\underline{M}_{0}-\underline{r}_{B_{R}} \times \underline{F}
$$

where $\underline{M}_{B_{R}}$ is the total external moment about the origin of $B_{R}$-frame. The second term on the right-hand side can be calculated directly as

$$
\begin{aligned}
\underline{r}_{B_{R}} \times \underline{F} & =\underline{r}_{B_{R}} \times\left[M \underline{\ddot{r}}_{B_{R}}+\sum_{j=1}^{k}\left(\dot{m}_{j} \dot{\underline{r}}_{m_{j}}+m_{j} \ddot{\underline{r}}_{m_{j}}\right)-\dot{m} \underline{V}_{0}\right] \\
& =\underline{r}_{B_{R}} \times M \underline{\ddot{r}}_{B_{R}}+\underline{r}_{B_{R}} \times \sum_{j=1}^{k}\left(\dot{m}_{j} \underline{\dot{\underline{r}}}_{m_{j}}+m_{j} \ddot{\underline{r}}_{m_{j}}\right)-\underline{r}_{B_{R}} \times \dot{m} \underline{V}_{0}
\end{aligned}
$$

where $\underline{F}$ is substituted from Eq. (3.9). To facilitate the subtraction in Eq. (4.5), Eq. (4.4) can be rewritten by replacing the vectors $\underline{r}_{i}, \underline{r}_{m_{j}}$, and $\underline{r}_{R}$ with their expansions as $\underline{r}_{B_{R}}+$ 
$\left.\underline{\rho}_{i}\right),\left(\underline{r}_{B_{R}}+\underline{\rho}_{m_{j}}\right)$, and $\left(\underline{r}_{B_{R}}+\underline{\rho}_{R}\right)$, respectively (Fig. 3.1). This results in a moment equation of the form

$$
\begin{aligned}
\underline{M}_{0}= & \sum_{i=1}^{n}\left(\underline{r}_{B_{R}}+\underline{\rho}_{i}\right) \times M_{i} \underline{\ddot{r}}_{i}+\sum_{j=1}^{k}\left(\underline{r}_{B_{R}}+\underline{\rho}_{m_{j}}\right) \times\left(\dot{m}_{j} \underline{\dot{r}}_{m_{j}}+m_{j} \ddot{\underline{r}}_{m_{j}}\right) \\
& -\left(\underline{r}_{B_{R}}+\underline{\rho}_{R}\right) \times \dot{m} \underline{V}_{0}
\end{aligned}
$$

By substituting Eqs. (4.6), (4.7), and the $\underline{V}_{0}$ expression from Eq. (3.11) into Eq. (4.5), while additionally using the fact that $\sum_{i=1}^{n} M_{i} \ddot{\ddot{r}}_{i}=M \underline{\ddot{r}}_{B_{R}}$, the rotational dynamics equation in terms of the moment of the external forces about the origin of the receiver's body frame is obtained as

$$
\begin{aligned}
\underline{M}_{B_{R}} & =\sum_{i=1}^{n} \underline{\rho}_{i} \times M_{i}\left(\ddot{\underline{\underline{r}}}_{B_{R}}+\underline{\ddot{g}}_{i}\right) \\
& +\sum_{j=1}^{k} \underline{\rho}_{m_{j}} \times\left[\dot{m}_{j}\left(\underline{\dot{r}}_{B_{R}}+\underline{\dot{\rho}}_{m_{j}}\right)+m_{j}\left(\underline{\ddot{\underline{r}}}_{B_{R}}+\ddot{\ddot{\rho}}_{m_{j}}\right)\right] \\
& -\underline{\rho}_{R} \times \dot{m}\left(\underline{\underline{r}}_{B_{T}}+\underline{V}_{\dot{m}}\right)
\end{aligned}
$$

Note that position vectors are written in terms of the vectorial sum of $\underline{r}_{B_{R}}$ and the respective position vector relative to $B_{R}$-frame. In this form of the rotational dynamic equation, the position vectors of ' $n$ ' and ' $k$ ' points and the receptacle are all relative to $B_{R}$-frame (see Fig. 3.1). Further, substituting $\underline{\underline{r}}_{B_{R}}$ and $\ddot{\underline{r}}_{B_{R}}$ from Eqs. (3.2) and (3.15), respectively, into Eq. (4.8) yields the rotational dynamics with explicit wind terms as

$$
\begin{aligned}
\underline{M}_{B_{R}} & =\sum_{i=1}^{n} \underline{\rho}_{i} \times M_{i}\left(\underline{\dot{U}}+\underline{\dot{W}}+\underline{\ddot{\rho}}_{i}\right) \\
& +\sum_{j=1}^{k} \underline{\rho}_{m_{j}} \times\left[\dot{m}_{j}\left(\underline{U}+\underline{W}+\underline{\dot{\rho}}_{m_{j}}\right)+m_{j}\left(\underline{\dot{U}}+\underline{\dot{W}}+\underline{\ddot{\rho}}_{m_{j}}\right)\right] \\
& -\underline{\rho}_{R} \times \dot{m}\left(\underline{\dot{r}}_{B_{T}}+\underline{V}_{\dot{m}}\right)
\end{aligned}
$$

Note that in the special case when $\dot{m}=\dot{m}_{j}=0$, both types of masses at ' $n$ ' points and ' $k$ ' points have the same effect in the rotational dynamics and the equation becomes the rotational dynamics of a system of $n+k$ concentrated masses. 
Recall that the external force acting on the receiver is due to the sources of (i) gravity, (ii) aerodynamics, and (iii) propulsion. The total moment of the external forces about the origin of $B_{R}$-frame can be written as

$$
\underline{M}_{B_{R}}=\underline{M}_{G}+\underline{M}_{A}+\underline{M}_{P}
$$

where $\underline{M}_{G}, \underline{M}_{A}$, and $\underline{M}_{P}$ are the moment vectors due to gravity, aerodynamics, and propulsion, respectively. Note that the moment due to gravity is not zero since the origin of $B_{R}$-frame is not necessarily at the $\mathrm{CM}$ of the receiver. However, the $\mathrm{CM}$ of the rigid body particles is, by definition, the origin of $B_{R}$-frame. Therefore, the gravitational moment is only due to the masses concentrated at ' $k$ ' points, such that

$$
\underline{M}_{G}=\sum_{j=1}^{k} \underline{\rho}_{m_{j}} \times m_{j} \underline{G}
$$

where $\underline{G}$ is the vector of the gravitational acceleration. The aerodynamic moment contains the aerodynamic rolling, pitching, and yawing moments, respectively, in the standard sense. The moment due to propulsion is

$$
\underline{M}_{P}=\underline{\rho}_{E} \times \underline{P}_{R}
$$

where $\underline{\rho}_{E}$ is the position vector of the application point of thrust relative to the origin of $B_{R}$-frame and $\underline{P}_{R}$ is the thrust vector. 


\section{CHAPTER 5}

\section{DERIVATION OF THE EQUATIONS IN MATRIX FORM}

In the preceding chapter, the translational and rotational equations of motion are derived in vector form. In this chapter, these equations are converted into state-space form, i.e. the first order matrix differential equations. Matrix equations are more suitable for implementation in simulation software such as MATLAB/Simulink. Further, statespace representation is the preferred form of system equations in control theory. The matrix form of the equations make use of vector representations in a specified frame instead of vectors. Since there are multiple reference frames, a given vector is chosen to be represented in the most convenient frame. Thus, the representations of vectors in various frames are used in the matrix form.

\subsection{Translational Kinematics}

Translational kinematics is derived in vector form in Eq. (3.3) as

$$
\underline{\dot{\xi}}=\underline{U}+\underline{W}-\underline{\dot{r}}_{B_{T}}
$$

Since $\underline{\xi}$ is the position of the receiver relative to the tanker and the motion of the receiver relative to the tanker can be expressed easily in $B_{T^{-}}$frame, vector $\underline{\xi}$ is expressed in $B_{T^{-}}$ frame, namely $\underline{\xi}=\left[\underline{\hat{B}}_{T}\right]^{T} \xi$. Note that the time derivative of $\xi$ in Eq. (3.3) is with respect to the inertial frame. Thus,

$$
\underline{\dot{\xi}}=[\underline{\dot{\xi}}]_{B_{T}}+\underline{\omega}_{B_{T}} \times \underline{\xi}
$$


where $[\underline{\dot{\xi}}]_{B_{T}}$ is the derivative of $\underline{\xi}$ with respect to $B_{T}$-frame and $\underline{\omega}_{B_{T}}$ is the angular velocity vector of $B_{T}$-frame with respect to the inertial frame. Then, using the property of vector product introduced in Eq. (2.15),

$$
\underline{\dot{\xi}}=\left[\underline{\hat{B}}_{T}\right]^{T} \dot{\xi}-\left[\underline{B}_{T}\right]^{T} \mathbf{S}\left(\omega_{B_{T}}\right) \xi
$$

where $\omega_{B_{T}}$ is the representation of $\underline{\omega}_{B_{T}}$ in $B_{T}$-frame. For the three velocity vectors on the right hand side of Eq. (3.3), three different frames are used to define the representations. The representation of $\underline{U}$ in $W_{R}$-frame is used, i.e. $\underline{U}=\left[\underline{\hat{W}}_{R}\right]^{T} U$. The wind vector, $\underline{W}$, is expressed in $B_{R}$-frame as $\underline{W}=\left[\underline{\hat{B}}_{R}\right]^{T} W$. The velocity vector of the tanker, $\underline{\dot{r}}_{B_{T}}$, is expressed in the inertial frame as $\underline{\dot{r}}_{B_{T}}=[\underline{\hat{I}}]^{T} \dot{r}_{B_{T}}$. Then, substituting $\underline{\dot{\xi}}$ from Eq. (5.2), Eq. (3.3) is rewritten as

$$
\left[\underline{\hat{B}}_{T}\right]^{T}\left[\dot{\xi}-\mathbf{S}\left(\omega_{B_{T}}\right) \xi\right]=\left[\underline{\hat{W}}_{R}\right]^{T} U+\left[\underline{\hat{B}}_{R}\right]^{T} W-[\underline{\hat{I}}]^{T} \dot{r}_{B_{T}}
$$

This equation will yield the matrix form of the translational dynamic equation once the vectrices are eliminated. To do this, all the terms should be written in terms of the same vectrix. Note that $\left[\underline{\hat{B}}_{R}\right]=\mathbf{R}_{\mathbf{B}_{\mathbf{R}} \mathbf{B}_{\mathbf{T}}}\left[\underline{\hat{B}}_{T}\right],\left[\underline{\hat{B}}_{T}\right]=\mathbf{R}_{\mathbf{B}_{\mathbf{R}} \mathbf{B}_{\mathbf{T}}}^{\mathbf{R}} \mathbf{R}_{\mathbf{B}_{\mathbf{R}} \mathbf{W}_{\mathbf{R}}}\left[\underline{\hat{W}}_{R}\right]$, and $\left[\underline{\hat{B}}_{T}\right]=\mathbf{R}_{\mathbf{B}_{\mathbf{T}} \mathbf{I}}[\underline{\hat{I}}]$. Equation (5.3) now becomes

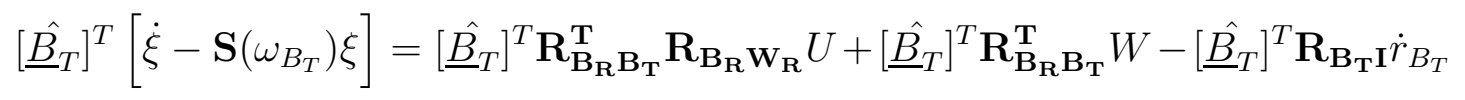

which, after canceling the common vectrices, leads to the matrix form of the translational dynamics as

$$
\dot{\xi}=\mathbf{R}_{\mathbf{B}_{\mathbf{R}} \mathbf{B}_{\mathbf{T}}}^{\mathbf{T}} \mathbf{R}_{\mathbf{B}_{\mathbf{R}} \mathbf{W}_{\mathbf{R}}} U+\mathbf{R}_{\mathbf{B}_{\mathbf{R}} \mathbf{B}_{\mathbf{T}}}^{\mathbf{T}} W-\mathbf{R}_{\mathbf{B}_{\mathbf{T}} \mathbf{I}} \dot{r}_{B_{T}}+\mathbf{S}\left(\omega_{\mathbf{B}_{\mathbf{T}}}\right) \xi
$$




\subsection{Rotational Kinematics}

As in the case of translational motion, the rotational motion of the receiver aircraft is formulated relative to the tanker aircraft. Thus, the rotational kinematics in the form of Poisson's equation is

$$
\dot{\mathbf{R}}_{\mathrm{B}_{\mathbf{R}} \mathrm{B}_{\mathrm{T}}}=\mathbf{S}\left(\omega_{\mathrm{B}_{\mathbf{R}} \mathrm{B}_{\mathrm{T}}}\right) \mathbf{R}_{\mathrm{B}_{\mathbf{R}} \mathrm{B}_{\mathrm{T}}}
$$

where note that both orientation and angular velocity of the receiver are relative to the tanker. Specifically, $\mathbf{R}_{\mathbf{B}_{\mathbf{R}} \mathbf{B}_{\mathbf{T}}}$ is the orientation of the receiver relative to the tanker. Further, $\omega_{B_{R} B_{T}}$ is the representation in $B_{R}$-frame of $\underline{\omega}_{B_{R} B_{T}}$, which is the angular velocity vector of the receiver relative to the tanker.

The angular velocity of the receiver relative to the inertial frame can be vectorially expanded as

$$
\underline{\omega}_{B_{R}}=\underline{\omega}_{B_{R} B_{T}}+\underline{\omega}_{B_{T}}
$$

where $\underline{\omega}_{B_{T}}$ is the angular velocity vector of the tanker relative to the inertial frame. The matrix form of this equation is derived as

$$
\omega_{B_{R}}=\omega_{B_{R} B_{T}}+\mathbf{R}_{\mathbf{B}_{\mathbf{R}} \mathbf{B}_{\mathbf{T}}} \omega_{B_{T}}
$$

where $\omega_{B_{R}}$ and $\omega_{B_{R} B_{T}}$ are the representations of the respective vectors in $B_{R}$-frame and $\omega_{B_{T}}$ is the representation of $\underline{\omega}_{B_{T}}$ in $B_{T}$-frame. Note that Eq. (5.6) is the standard form for the rotational kinematics equation. However, the rotation matrix and the angular velocity vector are not relative to a non-rotating and non-accelerating inertial frame. Appendix A proves that the equation still holds even in the case of a rotating and accelerating reference frame, while additionally deriving the expression for $\dot{\omega}_{B_{R}}$ as

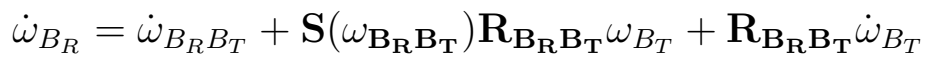




\subsection{Translational Dynamics}

The translational kinematic equation is written in Eq. (5.5) in terms of $U$ and $\mathbf{R}_{\mathbf{B}_{\mathbf{R}} \mathbf{W}_{\mathbf{R}}}$. Note that $U$ is the representation of the translational velocity of the receiver relative to the air in $W_{R}$-frame. Thus,

$$
U=\left[\begin{array}{c}
V_{R} \\
0 \\
0
\end{array}\right]
$$

where $V_{R}$ is the airspeed of the receiver. This is because the $x$-axis of $W_{R}$-frame, by definition, is along the velocity of the receiver relative to the air. Note further that $\mathbf{R}_{\mathbf{B}_{\mathbf{R}} \mathbf{W}_{\mathbf{R}}}$ is the rotation matrix from $W_{R}$-frame to $B_{R}$-frame and parameterized by the angle of attack, $\alpha_{R}$, and sideslip angle, $\beta_{R}$, of the receiver. In this section, the matrix form of the translational dynamic equation of Eq. (3.16) is written in terms of the triad $\left(V_{R}, \beta_{R}, \alpha_{R}\right)$, which defines the column matrix, $\mathcal{X}_{R}$, as

$$
\mathcal{X}_{R}=\left[V_{R} \beta_{R} \alpha_{R}\right]^{T}
$$

To derive the matrix form of the translational dynamics, every vector in Eq. (3.16) is written in terms of its representation using the vectrix formalism. All vectors will be

expressed with the vectrix of the inertial frame, $[\hat{I}]^{T}$, to enable the elimination of the vectrices from the equation. Recall that $\underline{U}=\left[\underline{\hat{W}}_{R}\right]^{T} U$ and can be expressed as

$$
\underline{U}=[\underline{\hat{I}}]^{T} \mathbf{R}_{\mathbf{B}_{\mathbf{T}} \mathbf{I}}^{\mathbf{T}} \mathbf{R}_{\mathbf{B}_{\mathbf{R}} \mathbf{B}_{\mathbf{T}}}^{\mathbf{T}} \mathbf{R}_{\mathbf{B}_{\mathbf{R}} \mathbf{W}_{\mathbf{R}}} U
$$

Then, since $[\hat{I}]^{T}$ is fixed, the derivative of $\underline{U}$ is found using chain rule to be

$$
\begin{aligned}
\dot{U}= & {[\underline{\hat{I}}]^{T}\left(\dot{\mathbf{R}}_{\mathbf{B}_{\mathbf{T}} \mathbf{I}}^{\mathbf{T}} \mathbf{R}_{\mathbf{B}_{\mathbf{R}} \mathbf{B}_{\mathbf{T}}}^{\mathbf{T}} \mathbf{R}_{\mathbf{B}_{\mathbf{R}} \mathbf{W}_{\mathbf{R}}} U+\mathbf{R}_{\mathbf{B}_{\mathbf{T}} \mathbf{I}}^{\mathbf{T}} \dot{\mathbf{R}}_{\mathbf{B}_{\mathbf{R}} \mathbf{B}_{\mathbf{T}}}^{\mathbf{T}} \mathbf{R}_{\mathbf{B}_{\mathbf{R}} \mathbf{W}_{\mathbf{R}} U+\mathbf{R}_{\mathbf{B}_{\mathbf{T}} \mathbf{I}}^{\mathbf{T}} \mathbf{R}_{\mathbf{B}_{\mathbf{R}} \mathbf{B}_{\mathbf{T}}}^{\mathbf{T}} \dot{\mathbf{R}}_{\mathbf{B}_{\mathbf{R}} \mathbf{W}_{\mathbf{R}}} U}\right.} \\
& \left.+\mathbf{R}_{\mathbf{B}_{\mathbf{T}} \mathbf{I}}^{\mathbf{T}} \mathbf{R}_{\mathbf{B}_{\mathbf{R}} \mathbf{B}_{\mathbf{T}}}^{\mathbf{T}} \mathbf{R}_{\mathbf{B}_{\mathbf{R}} \mathbf{W}_{\mathbf{R}}} \dot{U}\right)
\end{aligned}
$$


Matrix $\mathcal{E}_{R}$ is now defined such that

$$
\dot{\mathbf{R}}_{\mathbf{B}_{\mathbf{R}} \mathbf{W}_{\mathbf{R}}} U+\mathbf{R}_{\mathbf{B}_{\mathbf{R}} \mathbf{W}_{\mathbf{R}}} \dot{U}=\frac{\mathrm{d}}{\mathrm{d} t}\left(\mathbf{R}_{\mathbf{B}_{\mathbf{R}} \mathbf{w}_{\mathbf{R}}} U\right)=\frac{\mathrm{d}}{\mathrm{d} t}\left[\begin{array}{c}
V_{R} \cos \beta_{R} \cos \alpha_{R} \\
V_{R} \sin \beta_{R} \\
V_{R} \cos \beta_{R} \sin \alpha_{R}
\end{array}\right]=\mathcal{E}_{\mathbf{R}} \dot{\mathcal{X}}_{R}
$$

where

$$
\mathcal{E}_{\mathbf{R}}=\left[\begin{array}{ccc}
\cos \beta_{R} \cos \alpha_{R} & -V_{R} \sin \beta_{R} \cos \alpha_{R} & -V_{R} \cos \beta_{R} \sin \alpha_{R} \\
\sin \beta_{R} & V_{R} \cos \beta_{R} & 0 \\
\cos \beta_{R} \sin \alpha_{R} & -V_{R} \sin \beta_{R} \sin \alpha_{R} & V_{R} \cos \beta_{R} \cos \alpha_{R}
\end{array}\right]
$$

Further note, from Poisson's equation for the rotational kinematics of the tanker, that

$$
\dot{\mathbf{R}}_{\mathbf{B}_{\mathbf{T}} \mathbf{I}}=\mathbf{S}\left(\omega_{\mathbf{B}_{\mathbf{T}}}\right) \mathbf{R}_{\mathbf{B}_{\mathbf{T}} \mathbf{I}}^{\mathbf{T}}
$$

Using Eqs. (5.6), (5.14), and (5.16) in Eq. (5.13) yields

$$
\underline{\dot{U}}=[\underline{\hat{I}}]^{T} \mathbf{R}_{\mathbf{B}_{\mathbf{T}} \mathbf{T}}^{\mathbf{T}}\left\{-\left[\mathbf{R}_{\mathbf{B}_{\mathbf{R}} \mathbf{B}_{\mathbf{T}}}^{\mathbf{T}} \mathbf{S}\left(\omega_{\mathbf{B}_{\mathbf{R}} \mathbf{B}_{\mathbf{T}}}\right)+\mathbf{S}\left(\omega_{\mathbf{B}_{\mathbf{T}}}\right) \mathbf{R}_{\mathbf{B}_{\mathbf{R}} \mathbf{B}_{\mathbf{T}}}^{\mathbf{T}}\right] \mathbf{R}_{\mathbf{B}_{\mathbf{R}} \mathbf{W}_{\mathbf{R}}} U+\mathbf{R}_{\mathbf{B}_{\mathbf{R}} \mathbf{B}_{\mathbf{T}}}^{\mathbf{T}} \mathcal{E}_{\mathbf{R}} \dot{\mathcal{X}}_{R}\right\}
$$

Next, wind vector $\underline{W}$ and its derivative are expressed in the inertial frame. Recall that $\underline{W}=\left[\underline{\hat{B}}_{R}\right]^{T} W$ and can therefore be written as

$$
\underline{W}=[\underline{\underline{I}}]^{T} \mathbf{R}_{\mathbf{B}_{\mathbf{T}} \mathbf{I}}^{\mathbf{T}} \mathbf{R}_{\mathbf{B}_{\mathbf{R}} \mathbf{B}_{\mathbf{T}}}^{\mathbf{T}} W
$$

Taking the derivative of Eq. (5.18) using chain rule yields

$$
\underline{W}=[\underline{I}]^{T}\left(\dot{\mathbf{R}}_{\mathbf{B}_{\mathbf{T}} \mathbf{I}}^{\mathbf{T}} \mathbf{R}_{\mathbf{B}_{\mathbf{R}} \mathbf{B}_{\mathbf{T}}}^{\mathbf{T}} W+\mathbf{R}_{\mathbf{B}_{\mathbf{T}} \mathbf{I}}^{\mathbf{T}} \dot{\mathbf{R}}_{\mathbf{B}_{\mathbf{R}} \mathbf{B}_{\mathbf{T}}}^{\mathbf{T}} W+\mathbf{R}_{\mathbf{B}_{\mathbf{T}} \mathbf{I}}^{\mathbf{T}} \mathbf{R}_{\mathbf{B}_{\mathbf{R}} \mathbf{B}_{\mathbf{T}}}^{\mathbf{T}} \dot{W}\right)
$$

and using Eqs. (5.6) and (5.16) yields

$$
\underline{\dot{W}}=[\underline{\underline{I}}]^{T} \mathbf{R}_{\mathbf{B}_{\mathbf{T}}^{\mathbf{I}}}^{\mathbf{T}}\left\{-\left[\mathbf{R}_{\mathbf{B}_{\mathbf{R}} \mathbf{B}_{\mathbf{T}}}^{\mathbf{T}} \mathbf{S}\left(\omega_{\mathbf{B}_{\mathbf{R}} \mathbf{B}_{\mathbf{T}}}\right)+\mathbf{S}\left(\omega_{\mathbf{B}_{\mathbf{T}}}\right) \mathbf{R}_{\mathbf{B}_{\mathbf{R}} \mathbf{B}_{\mathbf{T}}}^{\mathbf{T}}\right] W+\mathbf{R}_{\mathbf{B}_{\mathbf{R}} \mathbf{B}_{\mathbf{T}}}^{\mathbf{T}} \dot{W}\right\}
$$

Two other vectors in the right hand side of Eq. (3.16), $\underline{F}$ and $\underline{\dot{r}}_{B_{T}}$, are expressed in the inertial frame, i.e. $\underline{F}=[\underline{\hat{I}}]^{T} F$ and $\underline{\dot{r}}_{B_{T}}=[\underline{\hat{I}}]^{T} \dot{r}_{B_{T}}$. For $\underline{V}_{\dot{m}}$, the most convenient 
frame is $B_{T}$-frame, i.e. $\underline{V}_{\dot{m}}=\left[\underline{\hat{B}}_{T}\right]^{T} V_{\dot{m}}$, which is expressed in the inertial frame as $\underline{V}_{\dot{m}}=[\underline{\hat{I}}]^{T} \mathbf{R}_{\mathbf{B}_{\mathbf{T}} \mathbf{I}}^{\mathbf{T}} V_{\dot{m}}$. The most convenient frame to express position vector $\underline{\rho}_{m_{j}}$ is obviously $B_{R}$-frame; thus, $\underline{\rho}_{m_{j}}=\left[\underline{\hat{B}}_{R}\right]^{T} \rho_{m_{j}}$. Then, the first derivative of $\underline{\rho}_{m_{j}}$ can be written as

$$
\underline{\underline{\rho}}_{m_{j}}=\left[\underline{\rho}_{m_{j}}\right]_{B_{R}}+\underline{\omega}_{B_{R}} \times \underline{\rho}_{m_{j}}
$$

where $\left[\underline{\rho}_{m_{j}}\right]_{B_{R}}$ and is the time derivative of $\underline{\rho}_{m_{j}}$ with respect to $B_{R}$-frame. It can be shown that, in vectrix formalism, Eq. (5.21) is rewritten as

$$
\underline{\dot{\rho}}_{m_{j}}=[\underline{\underline{I}}]^{T} \mathbf{R}_{\mathbf{B}_{\mathbf{T}} \mathbf{I}}^{\mathbf{T}} \mathbf{R}_{\mathbf{B}_{\mathbf{R}} \mathbf{B}_{\mathbf{T}}}^{\mathbf{T}}\left[\dot{\rho}_{m_{j}}-\mathbf{S}\left(\omega_{\mathbf{B}_{\mathbf{R}} \mathbf{B}_{\mathbf{T}}}\right) \rho_{m_{j}}-\mathbf{R}_{\mathbf{B}_{\mathbf{R}} \mathbf{B}_{\mathbf{T}}} \mathbf{S}\left(\omega_{\mathbf{B}_{\mathbf{T}}}\right) \mathbf{R}_{\mathbf{B}_{\mathbf{R}} \mathbf{B}_{\mathbf{T}} \rho_{m_{j}}}^{\mathbf{T}}\right]
$$

By taking the derivative of Eq. (5.21) using chain rule, the second derivative of $\underline{\rho}_{m_{j}}$ can be written as

$$
\begin{aligned}
\underline{\ddot{\rho}}_{m_{j}} & =\left[\underline{\ddot{\rho}}_{m_{j}}\right]_{B_{R}}+\underline{\omega}_{B_{R}} \times\left[\underline{\dot{\rho}}_{m_{j}}\right]_{B_{R}}+\underline{\dot{\omega}}_{B_{R}} \times \underline{\rho}_{m_{j}}+\underline{\omega}_{B_{R}} \times\left(\left[\underline{\rho}_{m_{j}}\right]_{B_{R}}+\underline{\omega}_{B_{R}} \times \underline{\rho}_{m_{j}}\right) \\
& =\left[\underline{\underline{\rho}}_{m_{j}}\right]_{B_{R}}+2 \underline{\omega}_{B_{R}} \times\left[\underline{\dot{\rho}}_{m_{j}}\right]_{B_{R}}+\underline{\dot{\omega}}_{B_{R}} \times \underline{\rho}_{m_{j}}+\underline{\omega}_{B_{R}} \times\left(\underline{\omega}_{B_{R}} \times \underline{\rho}_{m_{j}}\right)
\end{aligned}
$$

where $\left[\underline{\underline{\rho}}_{m_{j}}\right]_{B_{R}}$ is the second derivative of $\underline{\rho}_{m_{j}}$ with respect to $B_{R^{-}}$frame. It can be shown that, in vectrix formalism, Eq. (5.23) is rewritten as

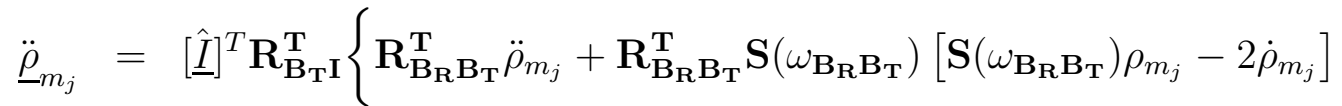

$$
\begin{aligned}
& +2 \mathbf{S}\left(\omega_{\mathbf{B}_{\mathbf{T}}}\right) \mathbf{R}_{\mathbf{B}_{\mathbf{R}} \mathbf{B}_{\mathbf{T}}}^{\mathbf{T}}\left[\mathbf{S}\left(\omega_{\mathbf{B}_{\mathbf{R}} \mathbf{B}_{\mathbf{T}}}\right) \rho_{m_{j}}-\dot{\rho}_{m_{j}}\right]
\end{aligned}
$$

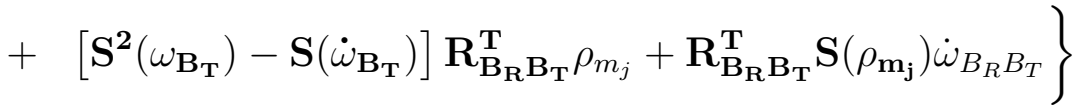

The details of the procedure to write $\underline{\dot{\rho}}_{m_{j}}$ and $\underline{\ddot{\rho}}_{m_{j}}$ in matrix form are given in App. B. 
Substituting all vectors written in vectrix form in Eq. (3.16) yields

$$
\begin{aligned}
& {[\underline{I}]^{T} \mathbf{R}_{\mathbf{B}_{\mathbf{T}} \mathbf{I}}^{\mathbf{T}}\left\{-\left[\mathbf{R}_{\mathbf{B}_{\mathbf{R}} \mathbf{B}_{\mathbf{T}}}^{\mathbf{T}} \mathbf{S}\left(\omega_{\mathbf{B}_{\mathbf{R}} \mathbf{B}_{\mathbf{T}}}\right)+\mathbf{S}\left(\omega_{\mathbf{B}_{\mathbf{T}}}\right) \mathbf{R}_{\mathbf{B}_{\mathbf{R}} \mathbf{B}_{\mathbf{T}}}^{\mathbf{T}}\right] \mathbf{R}_{\mathbf{B}_{\mathbf{R}} \mathbf{W}_{\mathbf{R}}} U+\mathbf{R}_{\mathbf{B}_{\mathbf{R}} \mathbf{B}_{\mathbf{T}}}^{\mathbf{T}} \mathcal{E}_{\mathbf{R}} \dot{\mathcal{X}}_{R}\right\}} \\
& =\frac{1}{(M+m)}\left[[\hat{I}]^{T} F-\dot{m}\left([\hat{I}]^{T} \mathbf{R}_{\mathbf{B}_{\mathbf{T}} \mathbf{I}}^{\mathbf{T}} \mathbf{R}_{\mathbf{B}_{\mathbf{R}} \mathbf{B}_{\mathbf{T}}}^{\mathbf{T}} \mathbf{R}_{\mathbf{B}_{\mathbf{R}} \mathbf{W}_{\mathbf{R}}} U+[\hat{I}]^{T} \mathbf{R}_{\mathbf{B}_{\mathbf{T}} \mathbf{I}}^{\mathbf{T}} \mathbf{R}_{\mathbf{B}_{\mathbf{R}} \mathbf{B}_{\mathbf{T}}}^{\mathbf{T}} W\right.\right. \\
& \left.\left.-[\underline{\hat{I}}]^{T} \dot{r}_{B_{T}}-[\underline{\hat{I}}]^{T} \mathbf{R}_{\mathbf{B}_{\mathbf{T}} \mathbf{I}}^{\mathbf{T}} V_{\dot{m}}\right)\right] \\
& -\frac{1}{(M+m)} \sum_{j=1}^{k} \dot{m}_{j}\left([ \hat { I } ] ^ { T } \mathbf { R } _ { \mathbf { B } _ { \mathbf { T } } \mathbf { T } } ^ { \mathbf { T } } \mathbf { R } _ { \mathbf { B } _ { \mathbf { R } } \mathbf { B } _ { \mathbf { T } } } ^ { \mathbf { T } } \left[\dot{\rho}_{m_{j}}-\mathbf{S}\left(\omega_{\mathbf{B}_{\mathbf{R}} \mathbf{B}_{\mathbf{T}}}\right) \rho_{m_{j}}\right.\right. \\
& \left.\left.-\mathbf{R}_{\mathbf{B}_{\mathbf{R}} \mathbf{B}_{\mathbf{T}}} \mathbf{S}\left(\omega_{\mathbf{B}_{\mathbf{T}}}\right) \mathbf{R}_{\mathbf{B}_{\mathbf{R}} \mathbf{B}_{\mathbf{T}}}^{\mathbf{T}} \rho_{m_{j}}\right]\right) \\
& +m_{j}\left([ \underline { \underline { I } } ] ^ { T } \mathbf { R } _ { \mathbf { B } _ { \mathbf { T } } \mathbf { I } } ^ { \mathbf { T } } \left\{\mathbf{R}_{\mathbf{B}_{\mathbf{R}} \mathbf{B}_{\mathbf{T}}}^{\mathbf{T}} \ddot{\rho}_{m_{j}}+\mathbf{R}_{\mathbf{B}_{\mathbf{R}} \mathbf{B}_{\mathbf{T}}}^{\mathbf{T}} \mathbf{S}\left(\omega_{\mathbf{B}_{\mathbf{R}} \mathbf{B}_{\mathbf{T}}}\right)\left[\mathbf{S}\left(\omega_{\mathbf{B}_{\mathbf{R}} \mathbf{B}_{\mathbf{T}}}\right) \rho_{m_{j}}-2 \dot{\rho}_{m_{j}}\right]\right.\right. \\
& +2 \mathbf{S}\left(\omega_{\mathbf{B}_{\mathbf{T}}}\right) \mathbf{R}_{\mathbf{B}_{\mathbf{R}} \mathbf{B}_{\mathbf{T}}}^{\mathbf{T}}\left[\mathbf{S}\left(\omega_{\mathbf{B}_{\mathbf{R}} \mathbf{B}_{\mathbf{T}}}\right) \rho_{m_{j}}-\dot{\rho}_{m_{j}}\right] \\
& \left.\left.+\left[\mathbf{S}^{2}\left(\omega_{\mathbf{B}_{\mathbf{T}}}\right)-\mathbf{S}\left(\dot{\omega}_{\mathbf{B}_{\mathbf{T}}}\right)\right] \mathbf{R}_{\mathbf{B}_{\mathbf{R}} \mathbf{B}_{\mathbf{T}}}^{\mathbf{T}} \rho_{m_{j}}+\mathbf{R}_{\mathbf{B}_{\mathbf{R}} \mathbf{B}_{\mathbf{T}}}^{\mathbf{T}} \mathbf{S}\left(\rho_{\mathbf{m}_{\mathbf{j}}}\right) \dot{\omega}_{B_{R} B_{T}}\right\}\right) \\
& -[\hat{I}]^{T} \mathbf{R}_{\mathbf{B}_{\mathbf{T}} \mathbf{I}}^{\mathbf{T}}\left\{-\left[\mathbf{R}_{\mathbf{B}_{\mathbf{R}} \mathbf{B}_{\mathbf{T}}}^{\mathbf{T}} \mathbf{S}\left(\omega_{\mathbf{B}_{\mathbf{R}} \mathbf{B}_{\mathbf{T}}}\right)+\mathbf{S}\left(\omega_{\mathbf{B}_{\mathbf{T}}}\right) \mathbf{R}_{\mathbf{B}_{\mathbf{R}} \mathbf{B}_{\mathbf{T}}}^{\mathbf{T}}\right] W+\mathbf{R}_{\mathbf{B}_{\mathbf{R}} \mathbf{B}_{\mathbf{T}}}^{\mathbf{T}} \dot{W}\right\}
\end{aligned}
$$

Canceling the common vectrix $[\hat{I}]$, pre-multiplying the entire equation by $\mathbf{R}_{\mathbf{B}_{\mathbf{R}} \mathbf{B}_{\mathbf{T}}} \mathbf{R}_{\mathbf{B}_{\mathbf{T}} \mathbf{I}}$, and using the identity in Eq. (2.22) results in

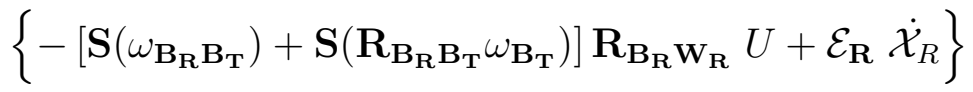

$$
\begin{aligned}
& =\frac{1}{(M+m)}\left[\mathbf{R}_{\mathbf{B}_{\mathbf{R}} \mathbf{B}_{\mathbf{T}}} \mathbf{R}_{\mathbf{B}_{\mathbf{T}} \mathbf{I}}\left(F+\dot{m} \dot{r}_{B_{T}}\right)-\dot{m}\left(\mathbf{R}_{\mathbf{B}_{\mathbf{R}} \mathbf{W}_{\mathbf{R}}} U+W-\mathbf{R}_{\mathbf{B}_{\mathbf{R}} \mathbf{B}_{\mathbf{T}}} V_{\dot{m}}\right)\right]
\end{aligned}
$$

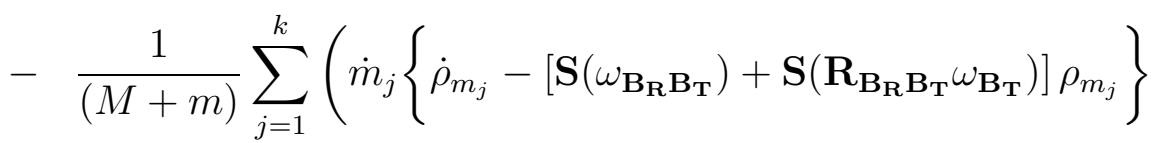

$$
\begin{aligned}
& +m_{j}\left\{\ddot{\rho}_{m_{j}}+\mathbf{S}\left(\omega_{\mathbf{B}_{\mathbf{R}} \mathbf{B}_{\mathbf{T}}}\right)\left[\mathbf{S}\left(\omega_{\mathbf{B}_{\mathbf{R}} \mathbf{B}_{\mathbf{T}}}\right) \rho_{m_{j}}-2 \dot{\rho}_{m_{j}}\right]\right. \\
& +2 \mathbf{S}\left(\mathbf{R}_{\mathbf{B}_{\mathbf{R}} \mathbf{B}_{\mathbf{T}}} \omega_{\mathbf{B}_{\mathbf{T}}}\right)\left[\mathbf{S}\left(\omega_{\mathbf{B}_{\mathbf{R}} \mathbf{B}_{\mathbf{T}}}\right) \rho_{m_{j}}-\dot{\rho}_{m_{j}}\right]
\end{aligned}
$$

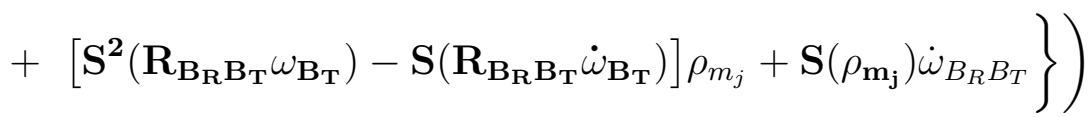

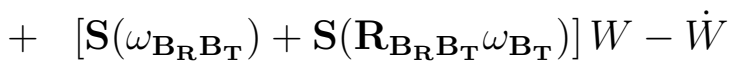


Rearranging to have $\dot{\mathcal{X}}_{R}$ on the left side and pre-multiplying by $\mathcal{E}_{\mathbf{R}}^{-1}$, the final form of the translational dynamics equation becomes

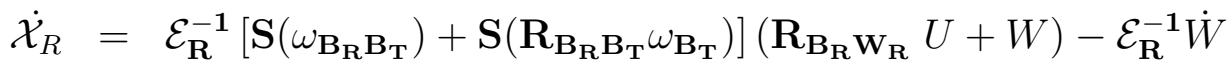

$$
\begin{aligned}
& +\frac{1}{(M+m)} \mathcal{E}_{\mathbf{R}}^{-\mathbf{1}}\left[\mathbf{R}_{\mathbf{B}_{\mathbf{R}} \mathbf{B}_{\mathbf{T}}} \mathbf{R}_{\mathbf{B}_{\mathbf{T}} \mathbf{I}}\left(F+\dot{m} \dot{r}_{B_{T}}\right)-\dot{m}\left(\mathbf{R}_{\mathbf{B}_{\mathbf{R}} \mathbf{W}_{\mathbf{R}}} U+W-\mathbf{R}_{\mathbf{B}_{\mathbf{R}} \mathbf{B}_{\mathbf{T}}} V_{\dot{m}}\right)\right]
\end{aligned}
$$

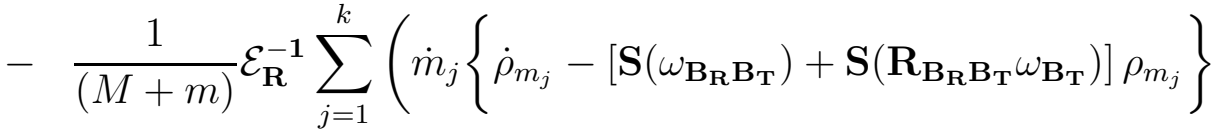

$$
\begin{aligned}
& +m_{j}\left\{\ddot{\rho}_{m_{j}}+\mathbf{S}\left(\omega_{\mathbf{B}_{\mathbf{R}} \mathbf{B}_{\mathbf{T}}}\right)\left[\mathbf{S}\left(\omega_{\mathbf{B}_{\mathbf{R}} \mathbf{B}_{\mathbf{T}}}\right) \rho_{m_{j}}-2 \dot{\rho}_{m_{j}}\right]\right. \\
& +2 \mathbf{S}\left(\mathbf{R}_{\mathbf{B}_{\mathbf{R}} \mathbf{B}_{\mathbf{T}}} \omega_{\mathbf{B}_{\mathbf{T}}}\right)\left[\mathbf{S}\left(\omega_{\mathbf{B}_{\mathbf{R}} \mathbf{B}_{\mathbf{T}}}\right) \rho_{m_{j}}-\dot{\rho}_{m_{j}}\right]
\end{aligned}
$$

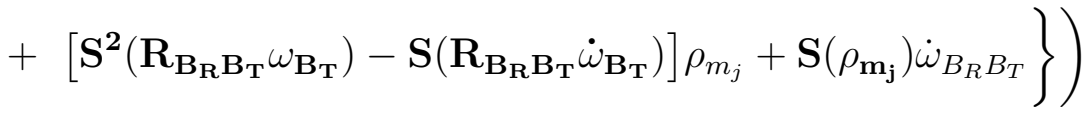

When regarded in this form, two additional observations may be made about the translational dynamics equation. First, the explicit dependency of the translational motion of the receiver aircraft on its rotational dynamics states and their time derivatives can be seen through the terms containing $\omega_{B_{R} B_{T}}$ and $\dot{\omega}_{B_{R} B_{T}}$. Secondly, the information about the motion of the tanker aircraft - both translational and rotational - are passed as exogenous inputs to the receiver aircraft. The variables included in this category are $\dot{r}_{B_{T}}, \mathbf{R}_{\mathbf{B}_{\mathbf{T}} \mathbf{I}}, \omega_{B_{T}}$ and $\dot{\omega}_{B_{T}}$.

\subsection{Rotational Dynamics}

Note that the rotational dynamic equation in Eq. (4.9) has the derivatives of $\underline{\rho}_{i}$ and $\underline{\rho}_{m_{j}}$. Recall that the first and second derivatives of $\underline{\rho}_{m_{j}}$ are given in Eqs. (5.21) and 
(5.23) in the previous section. Knowing that the $\underline{\rho}_{i}$ vectors are fixed in $B_{R}$-frame, Eqs. (5.21) and (5.23) may be extended to $\underline{\rho}_{i}$ as

$$
\begin{aligned}
\underline{\rho}_{i} & =\underline{\omega}_{B_{R}} \times \underline{\rho}_{i} \\
\underline{\underline{\rho}}_{i} & =\underline{\dot{\omega}}_{B_{R}} \times \underline{\rho}_{i}+\underline{\omega}_{B_{R}} \times \underline{\dot{\rho}}_{i} \\
& =\underline{\dot{\omega}}_{B_{R}} \times \underline{\rho}_{i}+\underline{\omega}_{B_{R}} \times\left(\underline{\omega}_{B_{R}} \times \underline{\rho}_{i}\right)
\end{aligned}
$$

Once the derivatives of $\underline{\rho}_{m_{j}}$ and $\underline{\rho}_{i}$ are substituted from Eqs. (5.21), (5.23), (5.28), and (5.29), Eq. (4.9) becomes

$$
\begin{aligned}
\underline{M}_{B_{R}}= & \sum_{i=1}^{n}\left\{\underline{\rho}_{i} \times M_{i}\left[\underline{\dot{U}}+\underline{\dot{W}}+\underline{\dot{\omega}}_{B_{R}} \times \underline{\rho}_{i}+\underline{\omega}_{B_{R}} \times\left(\underline{\omega}_{B_{R}} \times \underline{\rho}_{i}\right)\right]\right\} \\
+ & \sum_{j=1}^{k} \underline{\rho}_{m_{j}} \times\left\{\dot{m}_{j}\left(\underline{U}+\underline{W}+\left[\underline{\dot{\rho}}_{m_{j}}\right]_{B_{R}}+\underline{\omega} \times \underline{\rho}_{m_{j}}\right)\right. \\
& +m_{j}\left[\underline{\dot{U}}+\underline{\dot{W}}+\left[\underline{\rho}_{m_{j}}\right]_{B_{R}}+2 \underline{\omega}_{B_{R}} \times\left[\underline{\rho}_{m_{j}}\right]_{B_{R}}+\underline{\dot{\omega}}_{B_{R}} \times \underline{\rho}_{m_{j}}\right. \\
& \left.\left.+\underline{\omega}_{B_{R}} \times\left(\underline{\omega}_{B_{R}} \times \underline{\rho}_{m_{j}}\right)\right]\right\} \\
- & \underline{\rho}_{R} \times \dot{m}\left(\underline{\dot{\varphi}}_{B_{T}}+\underline{V}_{\dot{m}}\right)
\end{aligned}
$$

As done in the translational dynamics, the vectors in the above equation will be expressed in terms of vectrices to eventually derive the matrix form of the rotational dynamics. In doing so, each line of Eq. (5.30) is processed separately. The first line of Eq. (5.30) can be expanded as

$$
\sum_{i=1}^{n}\left\{\underline{\rho}_{i} \times M_{i}(\underline{\dot{U}}+\underline{\dot{W}})+M_{i} \underline{\rho}_{i} \times\left(\underline{\dot{\omega}}_{B_{R}} \times \underline{\rho}_{i}\right)+M_{i} \underline{\rho}_{i} \times\left[\underline{\omega}_{B_{R}} \times\left(\underline{\omega}_{B_{R}} \times \underline{\rho}_{i}\right)\right]\right\}
$$

It can be seen that the first term of this line is equal to zero after moving $(\underline{\dot{U}}+\underline{\dot{W}})$ outside the summation due to its independence of subscript $i$ as follows:

$$
\sum_{i=1}^{n} \underline{\rho}_{i} \times M_{i}(\underline{\dot{U}}+\underline{\dot{W}})=\left(\sum_{i=1}^{n} \underline{\rho}_{i} M_{i}\right) \times(\underline{\dot{U}}+\underline{\dot{W}})
$$


where $\sum_{i=1}^{n} \underline{\rho}_{i} M_{i}=0$ because the origin of $B_{R}$-frame is, by definition, at the CM of the constant masses $M_{i}$ at positions $\underline{\rho}_{i}$. Using the identities in Eqs. (2.11), (2.12), and (2.13), the second term of the expression in Eq. (5.31) can be simplified as

$$
\begin{aligned}
M_{i} \underline{\rho}_{i} \times\left(\underline{\dot{\omega}}_{B_{R}} \times \underline{\rho}_{i}\right) & =M_{i}\left[\left(\underline{\rho}_{i} \cdot \underline{\rho}_{i}\right) \underline{\dot{\omega}}_{B_{R}}-\left(\underline{\rho}_{i} \cdot \underline{\dot{\omega}}_{B_{R}}\right) \underline{\rho}_{i}\right] \\
& =\left[\underline{\hat{B}}_{R}\right]^{T}\left\{M_{i}\left[\left(\rho_{i}^{T} \rho_{i}\right) \mathbf{I}_{3 \times 3}-\left(\rho_{i} \rho_{i}^{T}\right)\right] \dot{\omega}_{B_{R}}\right\}
\end{aligned}
$$

where $\mathbf{I}_{\mathbf{3} \times \mathbf{3}}$ is an identity matrix included to ensure matrix dimension consistency. Moreover, the third term in Eq. (5.31) can be simplified using Eqs. (2.12) and (2.14)

$$
\begin{aligned}
M_{i} \underline{\rho}_{i} \times\left[\underline{\omega}_{B_{R}} \times\left(\underline{\omega}_{B_{R}} \times \underline{\rho}_{i}\right)\right] & =M_{i}\left[\left(\underline{\omega}_{B_{R}} \cdot \underline{\rho}_{i}\right) \underline{\rho}_{i} \times \underline{\omega}_{B_{R}}-\left(\underline{\omega}_{B_{R}} \cdot \underline{\omega}_{B_{R}}\right) \underline{\rho}_{i} \times \underline{\rho}_{i}\right] \\
& =M_{i}\left(\omega_{B_{R}}^{T} \rho_{i}\right) \underline{\rho}_{i} \times \underline{\omega}_{B_{R}}
\end{aligned}
$$

since $\underline{\rho}_{i} \times \underline{\rho}_{i}=0$. After rearranging this expression as $M_{i}\left(\rho_{i}^{T} \omega_{B_{R}}\right) \underline{\rho}_{i} \times \underline{\omega}_{B_{R}}$, the result can be further rewritten using the identity of Eq. (2.15) as

$$
M_{i}\left(\rho_{i}^{T} \omega_{B_{R}}\right) \underline{\rho}_{i} \times \underline{\omega}_{B_{R}}=-\left[\underline{\hat{B}}_{R}\right]^{T} M_{i}\left(\rho_{i}^{T} \omega_{B_{R}}\right) \mathbf{S}\left(\rho_{\mathbf{i}}\right) \omega_{B_{R}}
$$

which, when using the identity of Eq. (2.18), finally becomes

$$
\left[\underline{\hat{B}}_{R}\right]^{T} M_{i} \mathbf{S}\left(\omega_{\mathbf{B}_{\mathbf{R}}}\right)\left(\rho_{i} \rho_{i}^{T}\right) \omega_{B_{R}}
$$

By adding and subtracting $\left(\rho_{i}^{T} \rho_{i}\right) \omega_{B_{R}}$ and rearranging, Eq. (5.36) is further rewritten as

$$
-\left[\underline{\hat{B}}_{R}\right]^{T} \mathbf{S}\left(\omega_{\mathbf{B}_{\mathbf{R}}}\right)\left\{M_{i}\left[\left(\rho_{i}^{T} \rho_{i}\right) \mathbf{I}_{3 \times 3}-\rho_{i} \rho_{i}^{T}\right] \omega_{B_{R}}-M_{i}\left(\rho_{i}^{T} \rho_{i}\right) \omega_{B_{R}}\right\}
$$

where the second term is zero because $M_{i}\left(\rho_{i}^{T} \rho_{i}\right)$ is scalar and $\mathbf{S}\left(\omega_{\mathbf{B}_{\mathbf{R}}}\right) \omega_{B_{R}}$ is zero by Eq. (2.15). By substituting Eqs. (5.33) and (5.37) into Eq. (5.31), the first line can be rewritten as

$$
\left[\underline{\hat{B}}_{R}\right]^{T}\left\{\sum_{i=1}^{n} M_{i}\left[\left(\rho_{i}^{T} \rho_{i}\right) \mathbf{I}_{3 \times 3}-\left(\rho_{i} \rho_{i}^{T}\right)\right] \dot{\omega}_{B_{R}}-\mathbf{S}\left(\omega_{\mathbf{B}_{\mathbf{R}}}\right) \sum_{i=1}^{n} M_{i}\left[\left(\rho_{i}^{T} \rho_{i}\right) \mathbf{I}_{3 \times 3}-\rho_{i} \rho_{i}^{T}\right] \omega_{B_{R}}\right\}
$$


In both terms of this expression, the inertia matrix of the masses at $\underline{\rho}_{i}$ positions can be identified. Thus, the inertia matrix of the receiver, excluding fuel transferred, is defined as

$$
\underline{\underline{\mathbf{I}}}_{\mathbf{M}}=\sum_{i=1}^{n} M_{i}\left[\left(\rho_{i}^{T} \rho_{i}\right) \mathbf{I}_{3 \times 3}-\left(\rho_{i} \rho_{i}^{T}\right)\right]
$$

By substituting in Eq. (5.39), the first line of Eq. (5.30) can be further simplified to

$$
\left[\underline{\hat{B}}_{R}\right]^{T}\left[\underline{\underline{\mathbf{I}}}_{\mathbf{M}} \dot{\omega}_{B_{R}}-\mathbf{S}\left(\omega_{B_{R}}\right) \underline{\mathbf{I}}_{\mathbf{M}} \omega_{B_{R}}\right]
$$

As done in the first line of Eq. (5.30), the other lines can be rewritten as follows by using identities in Eqs. (2.11), (2.12), (2.13), and (2.14). The second line can be expanded as

$$
\sum_{j=1}^{k} \underline{\rho}_{m_{j}} \times \dot{m}_{j}(\underline{U}+\underline{W})+\underline{\rho}_{m_{j}} \times \dot{m}_{j}\left[\underline{\rho}_{m_{j}}\right]_{B_{R}}+\underline{\rho}_{m_{j}} \times \dot{m}_{j}\left(\underline{\omega} \times \underline{\rho}_{m_{j}}\right)
$$

the third term of which can be expanded using the identities in Eqs. (2.11), (2.12), and (2.13) similarly to Eq. (5.33) as

$$
\begin{aligned}
\underline{\rho}_{m_{j}} \times \dot{m}_{j}\left(\underline{\omega} \times \underline{\rho}_{m_{j}}\right) & =\dot{m}_{j}\left[\left(\underline{\rho}_{m_{j}} \cdot \underline{\rho}_{m_{j}}\right) \underline{\dot{\omega}}_{B_{R}}-\left(\underline{\rho}_{m_{j}} \cdot \underline{\dot{\omega}}_{B_{R}}\right) \underline{\rho}_{m_{j}}\right] \\
& =\left[\underline{B}_{R}\right]^{T}\left\{\dot{m}_{j}\left[\left(\rho_{m_{j}}^{T} \rho_{m_{j}}\right) \mathbf{I}_{3 \times 3}-\left(\rho_{m_{j}} \rho_{m_{j}}^{T}\right)\right] \dot{\omega}_{B_{R}}\right\}
\end{aligned}
$$

Substituting this expression into the second line and writing the other terms in vectrix notation results in the second line being rewritten as

$$
\begin{aligned}
& \sum_{j=1}^{k}\left\{\left[\underline{\hat{B}}_{R}\right]^{T} \rho_{m_{j}} \times \dot{m}_{j}\left(\left[\underline{\hat{W}}_{R}\right]^{T} U+\left[\underline{\hat{B}}_{R}\right]^{T} W\right)+\left[\underline{\hat{B}}_{R}\right]^{T} \rho_{m_{j}} \times \dot{m}_{j}\left[\underline{\hat{B}}_{R}\right]^{T} \dot{\rho}_{m_{j}}\right. \\
& \left.\quad+\dot{m}_{j}\left[\underline{\hat{B}}_{R}\right]^{T}\left[\left(\rho_{m_{j}}^{T} \rho_{m_{j}}\right) \mathbf{I}_{3 \times 3}-\left(\rho_{m_{j}} \rho_{m_{j}}^{T}\right)\right] \omega_{B_{R}}\right\}
\end{aligned}
$$

Further, using rotation matrices to write all vectors in $B_{R}$-frame results in

$$
\begin{aligned}
& \sum_{j=1}^{k}\left\{\left[\underline{\hat{B}}_{R}\right]^{T} \rho_{m_{j}} \times \dot{m}_{j}\left(\left[\underline{\hat{B}}_{R}\right]^{T} \mathbf{R}_{\mathbf{B}_{\mathbf{R}} \mathbf{W}_{\mathbf{R}}} U+\left[\underline{\hat{B}}_{R}\right]^{T} W\right)+\left[\underline{\hat{B}}_{R}\right]^{T} \rho_{m_{j}} \times \dot{m}_{j}\left[\underline{\hat{B}}_{R}\right]^{T} \dot{\rho}_{m_{j}}\right. \\
& \left.\quad+\dot{m}_{j}\left[\underline{\hat{B}}_{R}\right]^{T}\left[\left(\rho_{m_{j}}^{T} \rho_{m_{j}}\right) \mathbf{I}_{3 \times 3}-\left(\rho_{m_{j}} \rho_{m_{j}}^{T}\right)\right] \omega_{B_{R}}\right\}
\end{aligned}
$$


The third and fourth lines are processed by first expanding them to

$$
\begin{aligned}
& \sum_{j=1}^{k} \underline{\rho}_{m_{j}} \times m_{j}\left[\underline{\dot{U}}+\underline{\dot{W}}+\left[\underline{\tilde{\rho}}_{m_{j}}\right]_{B_{R}}+2 \underline{\omega}_{B_{R}} \times\left[\underline{\underline{\rho}}_{m_{j}}\right]_{B_{R}}+\underline{\dot{\omega}}_{B_{R}} \times \underline{\rho}_{m_{j}}\right. \\
& \left.\quad+\underline{\omega}_{B_{R}} \times\left(\underline{\omega}_{B_{R}} \times \underline{\rho}_{m_{j}}\right)\right] \\
& =\sum_{j=1}^{k} \underline{\rho}_{m_{j}} \times m_{j}(\underline{\dot{U}}+\underline{\dot{W}})+\underline{\rho}_{m_{j}} \times m_{j}\left[\underline{\ddot{\rho}}_{m_{j}}\right]_{B_{R}}+\underline{\rho}_{m_{j}} \times m_{j}\left(2 \underline{\omega}_{B_{R}} \times\left[\underline{\dot{\rho}}_{m_{j}}\right]_{B_{R}}\right) \\
& \left.\quad+\underline{\rho}_{m_{j}} \times m_{j}\left(\underline{\dot{\omega}}_{B_{R}} \times \underline{\rho}_{m_{j}}\right)+\underline{\rho}_{m_{j}} \times m_{j} \underline{\underline{\omega}}_{B_{R}} \times\left(\underline{\omega}_{B_{R}} \times \underline{\rho}_{m_{j}}\right)\right]
\end{aligned}
$$

The first line of this expansion can be rewritten by using the expressions that have already been developed for $\underline{\dot{U}}$ and $\underline{\dot{W}}$ in the translational dynamics matrix form derivation. Recall from Eqs. (5.17) and (5.20) that

$$
\underline{\dot{U}}=[\underline{\hat{I}}]^{T} \mathbf{R}_{\mathbf{B}_{\mathbf{T}} \mathbf{I}}^{\mathbf{T}}\left\{-\left[\mathbf{R}_{\mathbf{B}_{\mathbf{R}} \mathbf{B}_{\mathbf{T}}}^{\mathbf{T}} \mathbf{S}\left(\omega_{\mathbf{B}_{\mathbf{R}} \mathbf{B}_{\mathbf{T}}}\right)+\mathbf{S}\left(\omega_{\mathbf{B}_{\mathbf{T}}}\right) \mathbf{R}_{\mathbf{B}_{\mathbf{R}} \mathbf{B}_{\mathbf{T}}}^{\mathbf{T}}\right] \mathbf{R}_{\mathbf{B}_{\mathbf{R}} \mathbf{W}_{\mathbf{R}}} U+\mathbf{R}_{\mathbf{B}_{\mathbf{R}} \mathbf{B}_{\mathbf{T}}}^{\mathbf{T}} \mathcal{E}_{\mathbf{R}} \dot{\mathcal{X}}_{R}\right\}
$$

and

$$
\underline{\dot{W}}=[\underline{\hat{I}}]^{T} \mathbf{R}_{\mathbf{B}_{\mathbf{T}} \mathbf{I}}^{\mathbf{T}}\left\{-\left[\mathbf{R}_{\mathbf{B}_{\mathbf{R}} \mathbf{B}_{\mathbf{T}}}^{\mathbf{T}} \mathbf{S}\left(\omega_{\mathbf{B}_{\mathbf{R}} \mathbf{B}_{\mathbf{T}}}\right)+\mathbf{S}\left(\omega_{\mathbf{B}_{\mathbf{T}}}\right) \mathbf{R}_{\mathbf{B}_{\mathbf{R}} \mathbf{B}_{\mathbf{T}}}^{\mathbf{T}}\right]+\mathbf{R}_{\mathbf{B}_{\mathbf{R}} \mathbf{B}_{\mathbf{T}}}^{\mathbf{T}} \dot{W}\right\}
$$

Both of these derivatives may be written in $B_{R}$-frame by using the frame relations and rotation matrices. From the relation $[\underline{\hat{I}}]=\mathbf{R}_{\mathbf{B}_{\mathbf{T}} \mathbf{I}}^{\mathbf{T}} \mathbf{R}_{\mathbf{B}_{\mathbf{R}} \mathbf{B}_{\mathbf{T}}}^{\mathbf{T}}\left[\underline{\hat{B}}_{R}\right]$, it follows that

$$
[\underline{\hat{I}}]^{T}=\left[\underline{\hat{B}}_{R}\right]^{T} \mathbf{R}_{\mathbf{B}_{\mathbf{R}} \mathbf{B}_{\mathbf{T}}} \mathbf{R}_{\mathbf{B}_{\mathbf{T}} \mathbf{I}}
$$

By substitution of Eq. (5.46) and using the identity of Eq. (2.22), Eqs. (5.17) and (5.20) become

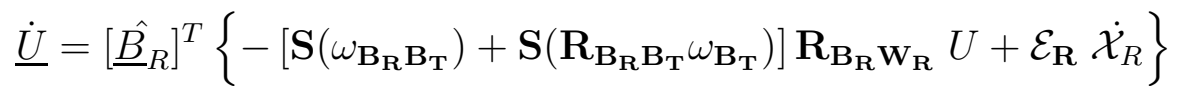

and

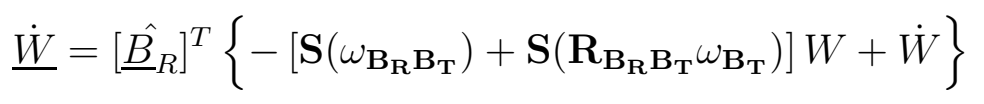


Making use of Eqs. (5.47) and (5.48), the first term of the expression in Eq. (5.45) may be written as

$$
\begin{aligned}
& \sum_{j=1}^{k} \underline{\rho}_{m_{j}} \times m_{j}(\underline{\dot{U}}+\underline{\dot{W}})
\end{aligned}
$$

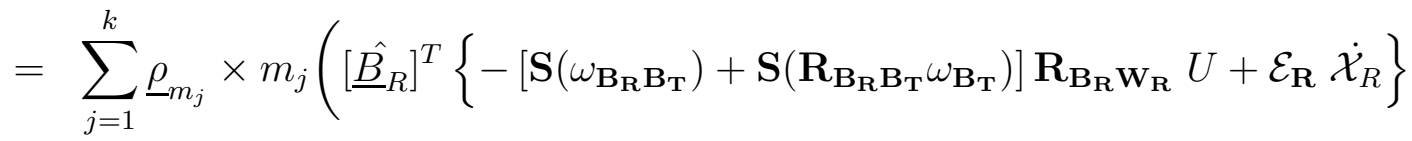

$$
\begin{aligned}
& \left.+\left[\underline{B}_{R}\right]^{T}\left\{-\left[\mathbf{S}\left(\omega_{\mathbf{B}_{\mathbf{R}} \mathbf{B}_{\mathbf{T}}}\right)+\mathbf{S}\left(\mathbf{R}_{\mathbf{B}_{\mathbf{R}} \mathbf{B}_{\mathbf{T}}} \omega_{\mathbf{B}_{\mathbf{T}}}\right)\right] W+\dot{W}\right\}\right) \\
& =\sum_{j=1}^{k} \underline{\rho}_{m_{j}} \times m_{j}\left([ \underline { \hat { B } } _ { R } ] ^ { T } \left\{-\left[\mathbf{S}\left(\omega_{\mathbf{B}_{\mathbf{R}} \mathbf{B}_{\mathbf{T}}}\right)+\mathbf{S}\left(\mathbf{R}_{\mathbf{B}_{\mathbf{R}} \mathbf{B}_{\mathbf{T}}} \omega_{\mathbf{B}_{\mathbf{T}}}\right)\right]\left(\mathbf{R}_{\mathbf{B}_{\mathbf{R}} \mathbf{W}_{\mathbf{R}}} U+W\right)+\mathcal{E}_{\mathbf{R}} \dot{\mathcal{X}}_{R}\right.\right. \\
& +\dot{W}\})
\end{aligned}
$$

The third and fourth terms of the expansion in Eq. (5.45) can be rewritten using the identities of Eqs. (2.11), (2.12), and (2.13) as

$$
\begin{aligned}
\underline{\rho}_{m_{j}} \times m_{j}\left(2 \underline{\omega}_{B_{R}} \times\left[\underline{\rho}_{m_{j}}\right]_{B_{R}}\right) & =m_{j}\left[\left(2 \underline{\rho}_{m_{j}} \cdot \underline{\dot{\rho}}_{m_{j}}\right) \underline{\omega}_{B_{R}}-\left(2 \underline{\rho}_{m_{j}} \cdot \underline{\omega}_{B_{R}}\right) \underline{\rho}_{m_{j}}\right] \\
& =\left[\underline{\hat{B}}_{R}\right]^{T}\left\{\dot{m}_{j}\left[\left(2 \rho_{m_{j}}^{T} \dot{\rho}_{m_{j}}\right) \mathbf{I}_{3 \times 3}-\left(2 \dot{\rho}_{m_{j}} \rho_{m_{j}}^{T}\right)\right] \omega_{B_{R}}\right\} \\
\underline{\rho}_{m_{j}} \times m_{j}\left(\underline{\dot{\omega}}_{B_{R}} \times \underline{\rho}_{m_{j}}\right) & =m_{j}\left[\left(\underline{\rho}_{m_{j}} \cdot \underline{\rho}_{m_{j}}\right) \underline{\dot{\omega}}_{B_{R}}-\left(\underline{\rho}_{m_{j}} \cdot \underline{\dot{\omega}}_{B_{R}}\right) \underline{\rho}_{m_{j}}\right] \\
& =\left[\underline{\hat{B}}_{R}\right]^{T}\left\{m_{j}\left[\left(\rho_{m_{j}}^{T} \rho_{m_{j}}\right) \mathbf{I}_{3 \times 3}-\left(\rho_{m_{j}} \rho_{m_{j}}^{T}\right)\right] \dot{\omega}_{B_{R}}\right\}
\end{aligned}
$$

The fifth term of the expression in Eq. (5.45) may be rewritten using Eqs. (2.12) and (2.14) as

$$
\begin{aligned}
\underline{\rho}_{m_{j}} \times m_{j}\left[\underline{\omega}_{B_{R}} \times\left(\underline{\omega}_{B_{R}} \times \underline{\rho}_{m_{j}}\right)\right] & =m_{j}\left[\left(\underline{\omega}_{B_{R}} \cdot \underline{\rho}_{m_{j}}\right) \underline{\rho}_{m_{j}} \times \underline{\omega}_{B_{R}}-\left(\underline{\omega}_{B_{R}} \cdot \underline{\omega}_{B_{R}}\right) \underline{\rho}_{m_{j}} \times \underline{\rho}_{m_{j}}\right] \\
& =m_{j}\left(\omega_{B_{R}}^{T} \rho_{m_{j}}\right) \underline{\rho}_{m_{j}} \times \underline{\omega}_{B_{R}}
\end{aligned}
$$


since $\underline{\rho}_{m_{j}} \times \underline{\rho}_{m_{j}}=0$. When these term expansions are substituted back into Eq. (5.45) and all vectors are written in vectrix notation, the third and fourth lines become

$$
\begin{aligned}
& \sum_{j=1}^{k}\left\{\left[\underline{\hat{B}}_{R}\right]^{T} \rho_{m_{j}} \times m_{j}\left([ \underline { \hat { B } } _ { R } ] ^ { T } \left\{-\left[\mathbf{S}\left(\omega_{\mathbf{B}_{\mathbf{R}} \mathbf{B}_{\mathbf{T}}}\right)+\mathbf{S}\left(\mathbf{R}_{\left.\left.\mathbf{B}_{\mathbf{R}} \mathbf{B}_{\mathbf{T}} \omega_{\mathbf{B}_{\mathbf{T}}}\right)\right]\left(\mathbf{R}_{\mathbf{B}_{\mathbf{R}} \mathbf{W}_{\mathbf{R}}} U+W\right)}\right.\right.\right.\right.\right. \\
& \left.\left.\quad+\mathcal{E}_{\mathbf{R}} \dot{\mathcal{X}}_{R}+\dot{W}\right\}\right)+\left[\underline{\hat{B}}_{R}\right]^{T} \rho_{m_{j}} \times m_{j}\left[\underline{\hat{B}}_{R}\right]^{T} \ddot{\rho}_{m_{j}} \\
& \quad+m_{j}\left[\underline{\hat{B}}_{R}\right]^{T}\left[\left(2 \rho_{m_{j}}^{T} \dot{\rho}_{m_{j}}\right) \mathbf{I}_{3 \times 3}-\left(2 \dot{\rho}_{m_{j}} \rho_{m_{j}}^{T}\right)\right] \omega_{B_{R}} \\
& \quad+m_{j}\left[\underline{\hat{B}}_{R}\right]^{T}\left[\left(\rho_{m_{j}}^{T} \rho_{m_{j}}\right) \mathbf{I}_{3 \times 3}-\left(\rho_{m_{j}} \rho_{m_{j}}^{T}\right)\right] \dot{\omega}_{B_{R}} \\
& \left.\quad+m_{j}\left(\omega_{B_{R}}^{T} \rho_{m_{j}}\right)\left[\underline{\hat{B}}_{R}\right]^{T} \rho_{m_{j}} \times\left[\underline{\hat{B}}_{R}\right]^{T} \omega_{B_{R}}\right\}
\end{aligned}
$$

The fifth line of Eq. (5.30) may be rewritten by first writing all vectors in vectrix notation and then using rotation matrices to write all vectors in $B_{R}$-frame. This results in

$$
\begin{aligned}
& \underline{\rho}_{R} \times \dot{m}\left(\underline{\dot{r}}_{B_{T}}+\underline{V}_{\dot{m}}\right)=-\left[\underline{\hat{B}}_{R}\right]^{T} \rho_{R} \times \dot{m}\left([\underline{\hat{I}}]^{T} \dot{r}_{B_{T}}+\left[\underline{\hat{B}}_{T}\right]^{T} V_{\dot{m}}\right)
\end{aligned}
$$

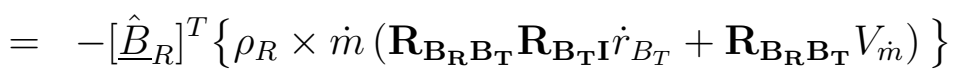

By additionally using the skew-symmetric operation of Eq. (2.15), each of the second, third/fourth, and fifth lines of Eq. (5.30) are further rewritten as follows:

Second line expressed in Eq. (5.44) becomes:

$$
\begin{aligned}
& {\left[\underline{\hat{B}}_{R}\right]^{T} \sum_{j=1}^{k}\left\{-\dot{m}_{j} \mathbf{S}\left(\rho_{m_{j}}\right)\left(\mathbf{R}_{\mathbf{B}_{\mathbf{R}} \mathbf{W}_{\mathbf{R}}} U+W\right)-\dot{m}_{j} \mathbf{S}\left(\rho_{m_{j}}\right) \dot{\rho}_{m_{j}}\right.} \\
& \left.+\dot{m}_{j}\left[\left(\rho_{m_{j}}^{T} \rho_{m_{j}}\right) \mathbf{I}_{3 \times 3}-\left(\rho_{m_{j}} \rho_{m_{j}}^{T}\right)\right] \omega_{B_{R}}\right\}
\end{aligned}
$$


Third and fourth lines expressed in Eq. (5.53) become:

$$
\begin{aligned}
& {\left[\underline{\hat{B}}_{R}\right]^{T} \sum_{j=1}^{k}\left\{-m_{j} \mathbf{S}\left(\rho_{m_{j}}\right)\left(-\left[\mathbf{S}\left(\omega_{\mathbf{B}_{\mathbf{R}} \mathbf{B}_{\mathbf{T}}}\right)+\mathbf{S}\left(\mathbf{R}_{\mathbf{B}_{\mathbf{R}} \mathbf{B}_{\mathbf{T}}} \omega_{\mathbf{B}_{\mathbf{T}}}\right)\right]\left(\mathbf{R}_{\mathbf{B}_{\mathbf{R}} \mathbf{W}_{\mathbf{R}}} U+W\right)\right.\right.} \\
& \left.+\mathcal{E}_{\mathbf{R}} \dot{\mathcal{X}}_{R}+\dot{W}\right)-m_{j} \mathbf{S}\left(\rho_{m_{j}}\right) \ddot{\rho}_{m_{j}} \\
& +m_{j}\left[\left(2 \rho_{m_{j}}^{T} \dot{\rho}_{m_{j}}\right) \mathbf{I}_{3 \times 3}-\left(2 \dot{\rho}_{m_{j}} \rho_{m_{j}}^{T}\right)\right] \omega_{B_{R}} \\
& +m_{j}\left[\left(\rho_{m_{j}}^{T} \rho_{m_{j}}\right) \mathbf{I}_{3 \times 3}-\left(\rho_{m_{j}} \rho_{m_{j}}^{T}\right)\right] \dot{\omega}_{B_{R}} \\
& \left.-m_{j}\left(\omega_{B_{R}}^{T} \rho_{m_{j}}\right) \mathbf{S}\left(\rho_{m_{j}}\right) \omega_{B_{R}}\right\}
\end{aligned}
$$

Fifth line expressed in Eq.(5.54) becomes:

$$
\left[\underline{\hat{B}}_{R}\right]^{T} \dot{m} \mathbf{S}\left(\rho_{R}\right)\left(\mathbf{R}_{\mathbf{B}_{\mathbf{R}} \mathbf{B}_{\mathbf{T}}} \mathbf{R}_{\mathbf{B}_{\mathbf{T}} \mathbf{I}} \dot{r}_{B_{T}}+\mathbf{R}_{\mathbf{B}_{\mathbf{R}} \mathbf{B}_{\mathbf{T}}} V_{\dot{m}}\right)
$$

When Eqs. (5.40), (5.55), (5.56), and (5.57) are substituted back into Eq. (5.30), the result is written as

$$
\begin{aligned}
& \underline{M}_{B_{R}}=\left[\underline{\hat{B}}_{R}\right]^{T}\left[\underline{\underline{\mathbf{I}}}_{\mathbf{M}} \dot{\omega}_{B_{R}}+\sum_{j=1}^{k} m_{j}\left[\left(\rho_{m_{j}}^{T} \rho_{m_{j}}\right) \mathbf{I}_{3 \times 3}-\left(\rho_{m_{j}} \rho_{m_{j}}^{T}\right)\right] \dot{\omega}_{B_{R}}-\mathbf{S}\left(\omega_{B_{R}}\right) \underline{\mathbf{I}}_{\mathbf{M}} \omega_{B_{R}}\right] \\
& +\left[\underline{\hat{B}}_{R}\right]^{T} \sum_{j=1}^{k}\left\{-\dot{m}_{j} \mathbf{S}\left(\rho_{m_{j}}\right)\left(\mathbf{R}_{\mathbf{B}_{\mathbf{R}} \mathbf{W}_{\mathbf{R}}} U+W\right)-\dot{m}_{j} \mathbf{S}\left(\rho_{m_{j}}\right) \dot{\rho}_{m_{j}}\right. \\
& \left.+\dot{m}_{j}\left[\left(\rho_{m_{j}}^{T} \rho_{m_{j}}\right) \mathbf{I}_{3 \times 3}-\left(\rho_{m_{j}} \rho_{m_{j}}^{T}\right)\right] \omega_{B_{R}}\right\} \\
& +\left[\underline{\hat{B}}_{R}\right]^{T} \sum_{j=1}^{k}\left\{-m_{j} \mathbf{S}\left(\rho_{m_{j}}\right)\left(-\left[\mathbf{S}\left(\omega_{\mathbf{B}_{\mathbf{R}} \mathbf{B}_{\mathbf{T}}}\right)+\mathbf{S}\left(\mathbf{R}_{\mathbf{B}_{\mathbf{R}} \mathbf{B}_{\mathbf{T}}} \omega_{\mathbf{B}_{\mathbf{T}}}\right)\right]\left(\mathbf{R}_{\mathbf{B}_{\mathbf{R}} \mathbf{W}_{\mathbf{R}}} U+W\right)\right.\right. \\
& \left.+\mathcal{E}_{\mathbf{R}} \dot{\mathcal{X}}_{R}+\dot{W}\right)-m_{j} \mathbf{S}\left(\rho_{m_{j}}\right) \ddot{\rho}_{m_{j}} \\
& +m_{j}\left[\left(2 \rho_{m_{j}}^{T} \dot{\rho}_{m_{j}}\right) \mathbf{I}_{3 \times 3}-\left(2 \dot{\rho}_{m_{j}} \rho_{m_{j}}^{T}\right)\right] \omega_{B_{R}} \\
& \left.-m_{j}\left(\omega_{B_{R}}^{T} \rho_{m_{j}}\right) \mathbf{S}\left(\rho_{m_{j}}\right) \omega_{B_{R}}\right\} \\
& +\left[\underline{\hat{B}}_{R}\right]^{T} \dot{m} \mathbf{S}\left(\rho_{R}\right)\left(\mathbf{R}_{\mathbf{B}_{\mathbf{R}} \mathbf{B}_{\mathbf{T}}} \mathbf{R}_{\mathbf{B}_{\mathbf{T}} \mathbf{I}} \dot{r}_{B_{T}}+\mathbf{R}_{\mathbf{B}_{\mathbf{R}} \mathbf{B}_{\mathbf{T}}} V_{\dot{m}}\right)
\end{aligned}
$$


where the fourth line of Eq. (5.56) is moved to the first line of Eq. (5.58). It is now useful to define the total inertia matrix of the entire system at a given time in $B_{R}$-frame as

$$
\underline{\underline{\mathbf{I}}}_{\mathbf{t}} \triangleq\left\{\underline{\mathbf{I}}_{\mathbf{M}}+\sum_{j=1}^{k} m_{j}\left[\left(\rho_{m_{j}}^{T} \rho_{m_{j}}\right) \mathbf{I}_{\mathbf{3} \times \mathbf{3}}-\rho_{m_{j}} \rho_{m_{j}}^{T}\right]\right\}
$$

which is always non-singular as $\underline{\underline{\mathbf{I}}}_{\mathbf{M}}$ is a mass property and $\rho_{m_{j}}^{T} \rho_{m_{j}}$ as well as $\rho_{m_{j}} \rho_{m_{j}}^{T}$ are positive definite terms. When Eq. (5.59) is substituted in Eq. (5.58), this yields

$$
\begin{aligned}
& \underline{M}_{B_{R}}=\left[\underline{\hat{B}}_{R}\right]^{T}\left[\underline{\underline{\mathbf{I}}}_{\mathrm{t}} \dot{\omega}_{B_{R}}-\mathbf{S}\left(\omega_{B_{R}}\right) \underline{\underline{\mathbf{I}}}_{\mathbf{M}} \omega_{B_{R}}\right] \\
& +\left[\underline{\hat{B}}_{R}\right]^{T} \sum_{j=1}^{k}\left\{-\dot{m}_{j} \mathbf{S}\left(\rho_{m_{j}}\right)\left(\mathbf{R}_{\mathbf{B}_{\mathbf{R}} \mathbf{W}_{\mathbf{R}}} U+W\right)-\dot{m}_{j} \mathbf{S}\left(\rho_{m_{j}}\right) \dot{\rho}_{m_{j}}\right. \\
& \left.+\dot{m}_{j}\left[\left(\rho_{m_{j}}^{T} \rho_{m_{j}}\right) \mathbf{I}_{3 \times 3}-\left(\rho_{m_{j}} \rho_{m_{j}}^{T}\right)\right] \omega_{B_{R}}\right\}
\end{aligned}
$$

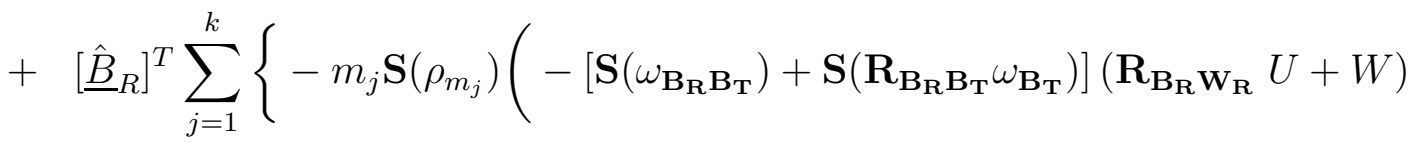

$$
\begin{aligned}
& \left.+\mathcal{E}_{\mathbf{R}} \dot{\mathcal{X}}_{R}+\dot{W}\right)-m_{j} \mathbf{S}\left(\rho_{m_{j}}\right) \ddot{\rho}_{m_{j}} \\
& +m_{j}\left[\left(2 \rho_{m_{j}}^{T} \dot{\rho}_{m_{j}}\right) \mathbf{I}_{3 \times 3}-\left(2 \dot{\rho}_{m_{j}} \rho_{m_{j}}^{T}\right)\right] \omega_{B_{R}} \\
& \left.-m_{j}\left(\omega_{B_{R}}^{T} \rho_{m_{j}}\right) \mathbf{S}\left(\rho_{m_{j}}\right) \omega_{B_{R}}\right\} \\
& +\left[\underline{\hat{B}}_{R}\right]^{T} \dot{m} \mathbf{S}\left(\rho_{R}\right)\left(\mathbf{R}_{\mathbf{B}_{\mathbf{R}} \mathbf{B}_{\mathbf{T}}} \mathbf{R}_{\mathbf{B}_{\mathbf{T}} \mathbf{I}} \dot{r}_{B_{T}}+\mathbf{R}_{\mathbf{B}_{\mathbf{R}} \mathbf{B}_{\mathbf{T}}} V_{\dot{m}}\right)
\end{aligned}
$$


Further substituting $\omega_{B_{R}}$ and $\dot{\omega}_{B_{R}}$ from Eqs. (5.8) and (5.9) and combining terms results in

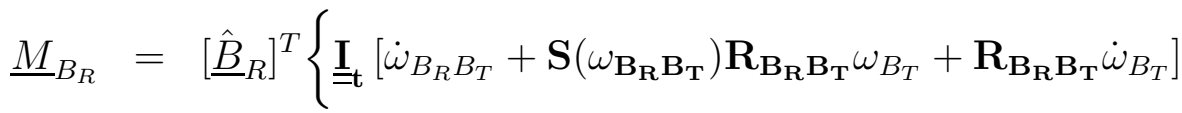

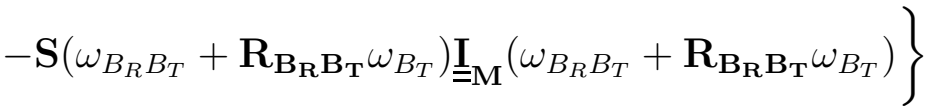

$$
\begin{aligned}
& +\left[\underline{\hat{B}}_{R}\right]^{T} \sum_{j=1}^{k} \dot{m}_{j}\left\{-\mathbf{S}\left(\rho_{m_{j}}\right)\left(\mathbf{R}_{\mathbf{B}_{\mathbf{R}} \mathbf{W}_{\mathbf{R}}} U+W\right)-\mathbf{S}\left(\rho_{m_{j}}\right) \dot{\rho}_{m_{j}}\right. \\
& \left.+\left[\left(\rho_{m_{j}}^{T} \rho_{m_{j}}\right) \mathbf{I}_{3 \times 3}-\left(\rho_{m_{j}} \rho_{m_{j}}^{T}\right)\right]\left(\omega_{B_{R} B_{T}}+\mathbf{R}_{\mathbf{B}_{\mathbf{R}} \mathbf{B}_{\mathbf{T}}} \omega_{B_{T}}\right)\right\}
\end{aligned}
$$

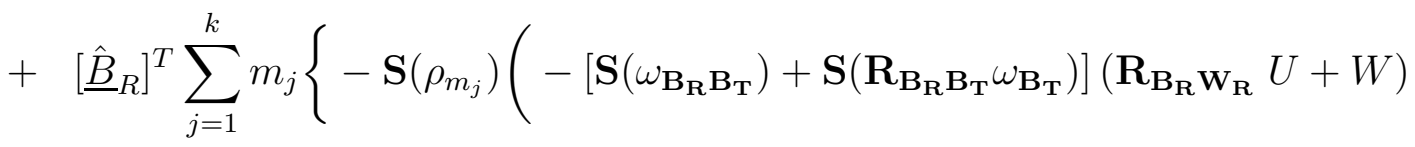

$$
\begin{aligned}
& \left.+\mathcal{E}_{\mathbf{R}} \dot{\mathcal{X}}_{R}+\dot{W}\right)-\mathbf{S}\left(\rho_{m_{j}}\right) \ddot{\rho}_{m_{j}} \\
& +\left[\left(2 \rho_{m_{j}}^{T} \dot{\rho}_{m_{j}}\right) \mathbf{I}_{3 \times 3}-\left(2 \dot{\rho}_{m_{j}} \rho_{m_{j}}^{T}\right)\right]\left(\omega_{B_{R} B_{T}}+\mathbf{R}_{\mathbf{B}_{\mathbf{R}} \mathbf{B}_{\mathbf{T}}} \omega_{B_{T}}\right)
\end{aligned}
$$

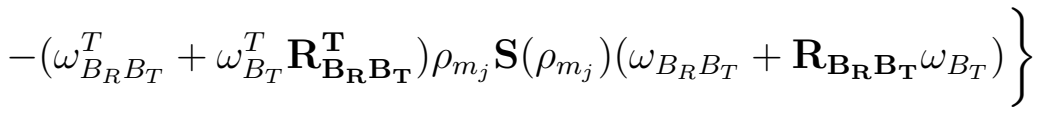

$$
\begin{aligned}
& +\left[\underline{\hat{B}}_{R}\right]^{T} \dot{m} \mathbf{S}\left(\rho_{R}\right)\left(\mathbf{R}_{\mathbf{B}_{\mathbf{R}} \mathbf{B}_{\mathbf{T}}} \mathbf{R}_{\mathbf{B}_{\mathbf{T}} \mathbf{I}} \dot{r}_{B_{T}}+\mathbf{R}_{\mathbf{B}_{\mathbf{R}} \mathbf{B}_{\mathbf{T}}} V_{\dot{m}}\right)
\end{aligned}
$$

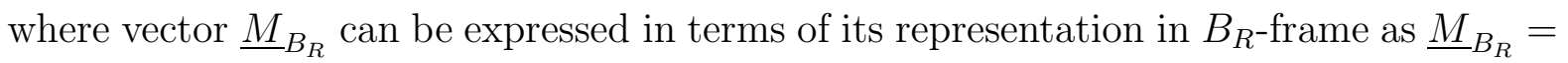
$\left[\underline{\hat{B}}_{R}\right]^{T} M_{B_{R}}$. Also note that $\left(\omega_{B_{R} B_{T}}^{T}+\omega_{B_{T}}^{T} \mathbf{R}_{\mathbf{B}_{\mathbf{R}} \mathbf{B}_{\mathbf{T}}}^{\mathbf{T}}\right) \rho_{m_{j}}$ in the second line from the end is a scalar and therefore can be moved after $\mathbf{S}\left(\rho_{m_{j}}\right)$. 
Canceling the common vectrices and further combining similar terms results in

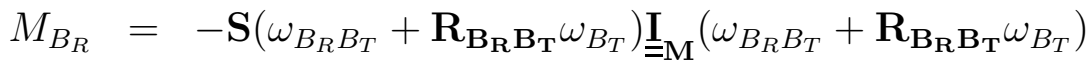

$$
\begin{aligned}
& -\sum_{j=1}^{k} \mathbf{S}\left(\rho_{\mathbf{m}_{\mathbf{j}}}\right)\left[m_{j}\left(\omega_{B_{R} B_{T}}^{T}+\omega_{B_{T}}^{T} \mathbf{R}_{\mathbf{B}_{\mathbf{R}} \mathbf{B}_{\mathbf{T}}}^{\mathbf{T}}\right) \rho_{m_{j}}\left(\omega_{B_{R} B_{T}}+\mathbf{R}_{\mathbf{B}_{\mathbf{R}} \mathbf{B}_{\mathbf{T}}} \omega_{B_{T}}\right)\right. \\
& \left.+m_{j} \ddot{\rho}_{m_{j}}+\dot{m}_{j} \dot{\rho}_{m_{j}}\right] \\
& -\left[\sum_{j=1}^{k} \mathbf{S}\left(\rho_{\mathbf{m}_{\mathbf{j}}}\right) m_{j}\right]\left\{-\left[\mathbf{S}\left(\omega_{\mathbf{B}_{\mathbf{R}} \mathbf{B}_{\mathbf{T}}}\right)+\mathbf{S}\left(\mathbf{R}_{\mathbf{B}_{\mathbf{R}} \mathbf{B}_{\mathbf{T}}} \omega_{\mathbf{B}_{\mathbf{T}}}\right)\right]\left(\mathbf{R}_{\mathbf{B}_{\mathbf{R}} \mathbf{W}_{\mathbf{R}}} U+W\right)\right. \\
& \left.+\mathcal{E}_{\mathbf{R}} \dot{\mathcal{X}}_{R}+\dot{W}\right\} \\
& -\left[\sum_{j=1}^{k} \mathbf{S}\left(\rho_{\mathbf{m}_{\mathbf{j}}}\right) \dot{m}_{j}\right]\left(\mathbf{R}_{\mathbf{B}_{\mathbf{R}} \mathbf{w}_{\mathbf{R}}} U+W\right) \\
& +2 \sum_{j=1}^{k} m_{j}\left[\left(\rho_{m_{j}}^{T} \dot{\rho}_{m_{j}}\right) \mathbf{I}_{\mathbf{3} \times \mathbf{3}}-\dot{\rho}_{m_{j}} \rho_{m_{j}}^{T}\right]\left(\omega_{B_{R} B_{T}}+\mathbf{R}_{\mathbf{B}_{\mathbf{R}} \mathbf{B}_{\mathbf{T}}} \omega_{B_{T}}\right) \\
& +\sum_{j=1}^{k} \dot{m}_{j}\left[\left(\rho_{m_{j}}^{T} \rho_{m_{j}}\right) \mathbf{I}_{\mathbf{3} \times \mathbf{3}}-\rho_{m_{j}} \rho_{m_{j}}^{T}\right]\left(\omega_{B_{R} B_{T}}+\mathbf{R}_{\mathbf{B}_{\mathbf{R}} \mathbf{B}_{\mathbf{T}}} \omega_{B_{T}}\right) \\
& +\underline{\mathbf{I}}_{\mathbf{t}}\left[\dot{\omega}_{B_{R} B_{T}}+\mathbf{S}\left(\omega_{\mathbf{B}_{\mathbf{R}} \mathbf{B}_{\mathbf{T}}}\right) \mathbf{R}_{\mathbf{B}_{\mathbf{R}} \mathbf{B}_{\mathbf{T}}} \omega_{B_{T}}+\mathbf{R}_{\mathbf{B}_{\mathbf{R}} \mathbf{B}_{\mathbf{T}}} \dot{\omega}_{B_{T}}\right] \\
& +\dot{m} \mathbf{S}\left(\rho_{R}\right)\left(\mathbf{R}_{\mathbf{B}_{\mathbf{R}} \mathbf{B}_{\mathbf{T}}} \mathbf{R}_{\mathbf{B}_{\mathbf{T}} \mathbf{I}} \dot{r}_{B_{T}}+\mathbf{R}_{\mathbf{B}_{\mathbf{R}} \mathbf{B}_{\mathbf{T}}} V_{\dot{m}}\right)
\end{aligned}
$$

When Eq. (5.62) is rearranged to have $\dot{\omega}_{B_{R} B_{T}}$ on the left side, the final form of the rotational dynamics equation is written as 


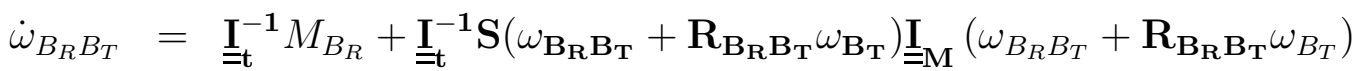

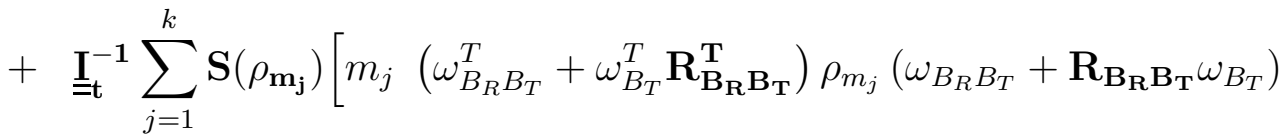

$$
\begin{aligned}
& \left.+m_{j} \ddot{\rho}_{m_{j}}+\dot{m}_{j} \dot{\rho}_{m_{j}}\right] \\
& +\underline{\mathbf{I}}_{\mathbf{t}}^{-\mathbf{1}}\left[\sum_{j=1}^{k} \mathbf{S}\left(\rho_{\mathbf{m}_{\mathbf{j}}}\right) m_{j}\right]\left\{-\left[\mathbf{S}\left(\omega_{\mathbf{B}_{\mathbf{R}} \mathbf{B}_{\mathbf{T}}}\right)+\mathbf{S}\left(\mathbf{R}_{\mathbf{B}_{\mathbf{R}} \mathbf{B}_{\mathbf{T}}} \omega_{\mathbf{B}_{\mathbf{T}}}\right)\right]\left(\mathbf{R}_{\mathbf{B}_{\mathbf{R}} \mathbf{W}_{\mathbf{R}}} U+W\right)\right. \\
& \left.+\mathcal{E}_{\mathbf{R}} \dot{\mathcal{X}}_{R}+\dot{W}\right\} \\
& +\underline{\mathbf{I}}_{\mathbf{t}}^{-\mathbf{1}}\left[\sum_{j=1}^{k} \mathbf{S}\left(\rho_{\mathbf{m}_{\mathbf{j}}}\right) \dot{m}_{j}\right] \quad\left(\mathbf{R}_{\mathbf{B}_{\mathbf{R}} \mathbf{w}_{\mathbf{R}}} U+W\right) \\
& -2 \underline{\underline{\mathbf{I}}}_{\mathbf{t}}^{-\mathbf{1}} \sum_{j=1}^{k} m_{j}\left[\left(\rho_{m_{j}}^{T} \dot{\rho}_{m_{j}}\right) \mathbf{I}_{\mathbf{3} \times \mathbf{3}}-\dot{\rho}_{m_{j}} \rho_{m_{j}}^{T}\right]\left(\omega_{B_{R} B_{T}}+\mathbf{R}_{\mathbf{B}_{\mathbf{R}} \mathbf{B}_{\mathbf{T}}} \omega_{B_{T}}\right) \\
& \text { - } \underline{\underline{\mathbf{I}}}_{\mathbf{t}}^{-\mathbf{1}} \sum_{j=1}^{k} \dot{m}_{j}\left[\left(\rho_{m_{j}}^{T} \rho_{m_{j}}\right) \mathbf{I}_{\mathbf{3} \times \mathbf{3}}-\rho_{m_{j}} \rho_{m_{j}}^{T}\right]\left(\omega_{B_{R} B_{T}}+\mathbf{R}_{\mathbf{B}_{\mathbf{R}} \mathbf{B}_{\mathbf{T}}} \omega_{B_{T}}\right)
\end{aligned}
$$

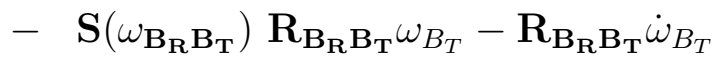

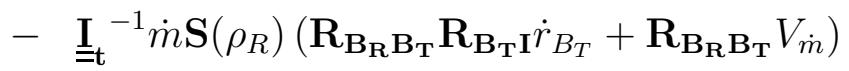

In this equation, all the variables are with respect to the body frame of the receiver or the body frame of the tanker with the exception of $\dot{r}_{B_{T}}$, which is the velocity of the tanker relative to the inertial frame. When the rotational dynamics is represented in the above form, it seems quite complicated. However, it is very suitable for the application of aerial refueling. Angular velocity and the orientation as well as the translational velocity and position of the receiver are defined with respect to $B_{T}$-frame. The inertia and mass properties of the receiver before the aerial refueling can be directly used in the equation. Effect of the refueling on the rotational dynamics is represented by the concentrated fuel mass and its CM location in each fuel tank. In the case of multiple fuel tanks, the effect of fuel flow into each tank can be taken into account separately. Since the equation is written with respect to a point fixed geometrically in the body of the 
receiver, change of the $\mathrm{CM}$ during the refueling is already incorporated in the equation. Another advantage of writing the equation with respect to a geometrically fixed frame in the body is seen when the aerodynamic variables such as airspeed, angle of attack and side-slip angle and aerodynamic stability derivatives need to be used. Since these variables and derivatives are determined by the geometric shape of the aircraft, not its mass properties, the standard definitions can be directly used without any modification or re-interpretation. 


\section{CHAPTER 6}

\section{STATE-SPACE FORM OF THE DYNAMICS EQUATIONS}

The most general forms of the dynamics equations from Eqs. (5.27) and (5.63) are

$$
\begin{aligned}
\dot{\mathcal{X}}_{R} & =\mathbf{f}_{\mathbf{1}} \dot{\omega}_{B_{R} B_{T}}+c_{1} \\
\dot{\omega}_{B_{R} B_{T}} & =\mathbf{f}_{\mathbf{2}} \dot{\mathcal{X}}_{R}+c_{2}
\end{aligned}
$$

where

$$
\begin{aligned}
& \mathbf{f}_{\mathbf{1}}=-\frac{1}{(M+m)} \mathcal{E}_{\mathbf{R}}^{-\mathbf{1}}\left[\sum_{j=1}^{k} m_{j} \mathbf{S}\left(\rho_{\mathbf{m}_{\mathbf{j}}}\right)\right]
\end{aligned}
$$

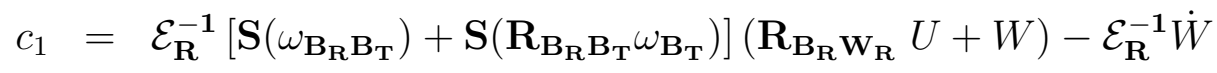

$$
\begin{aligned}
& +\frac{1}{(M+m)} \mathcal{E}_{\mathbf{R}}^{-\mathbf{1}}\left[\mathbf{R}_{\mathbf{B}_{\mathbf{R}} \mathbf{B}_{\mathbf{T}}} \mathbf{R}_{\mathbf{B}_{\mathbf{T}} \mathbf{I}}\left(F+\dot{m} \dot{r}_{B_{T}}\right)-\dot{m}\left(\mathbf{R}_{\mathbf{B}_{\mathbf{R}} \mathbf{W}_{\mathbf{R}}} U+W-\mathbf{R}_{\mathbf{B}_{\mathbf{R}} \mathbf{B}_{\mathbf{T}}} V_{\dot{m}}\right)\right]
\end{aligned}
$$

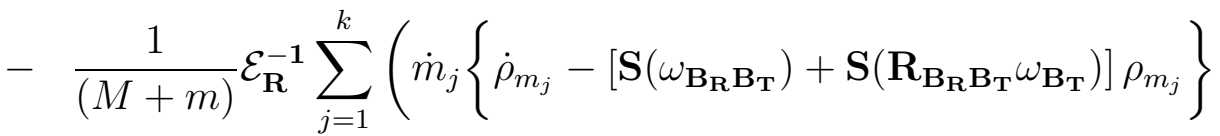

$$
\begin{aligned}
& +m_{j}\left\{\ddot{\rho}_{m_{j}}+\mathbf{S}\left(\omega_{\mathbf{B}_{\mathbf{R}} \mathbf{B}_{\mathbf{T}}}\right)\left[\mathbf{S}\left(\omega_{\mathbf{B}_{\mathbf{R}} \mathbf{B}_{\mathbf{T}}}\right) \rho_{m_{j}}-2 \dot{\rho}_{m_{j}}\right]\right.
\end{aligned}
$$

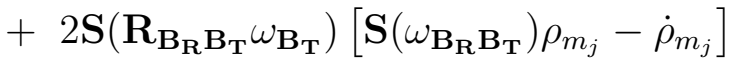

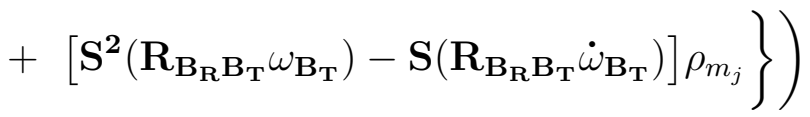

$$
\begin{aligned}
& \mathbf{f}_{\mathbf{2}}=\underline{\underline{\mathbf{I}}}_{\mathbf{t}}^{-\mathbf{1}}\left[\sum_{j=1}^{k} \mathbf{S}\left(\rho_{\mathbf{m}_{\mathbf{j}}}\right) m_{j}\right] \mathcal{E}_{\mathbf{R}}
\end{aligned}
$$


and

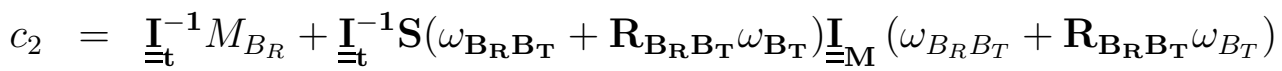

$$
\begin{aligned}
& +\underline{\mathbf{I}}_{=}^{-\mathbf{1}} \sum_{j=1}^{k} \mathbf{S}\left(\rho_{\mathbf{m}_{\mathbf{j}}}\right)\left[m_{j}\left(\omega_{B_{R} B_{T}}^{T}+\omega_{B_{T}}^{T} \mathbf{R}_{\mathbf{B}_{\mathbf{R}} \mathbf{B}_{\mathbf{T}}}^{\mathbf{T}}\right) \rho_{m_{j}}\left(\omega_{B_{R} B_{T}}+\mathbf{R}_{\mathbf{B}_{\mathbf{R}} \mathbf{B}_{\mathbf{T}}} \omega_{B_{T}}\right)\right. \\
& \left.+m_{j} \ddot{\rho}_{m_{j}}+\dot{m}_{j} \dot{\rho}_{m_{j}}\right] \\
& +\underline{\mathbf{I}}_{\mathbf{t}}^{-\mathbf{1}}\left[\sum_{j=1}^{k} \mathbf{S}\left(\rho_{\mathbf{m}_{\mathbf{j}}}\right) m_{j}\right]\left\{-\left[\mathbf{S}\left(\omega_{\mathbf{B}_{\mathbf{R}} \mathbf{B}_{\mathbf{T}}}\right)+\mathbf{S}\left(\mathbf{R}_{\mathbf{B}_{\mathbf{R}} \mathbf{B}_{\mathbf{T}}} \omega_{\mathbf{B}_{\mathbf{T}}}\right)\right]\left(\mathbf{R}_{\mathbf{B}_{\mathbf{R}} \mathbf{W}_{\mathbf{R}}} U+W\right)\right. \\
& +\dot{W}\} \\
& +\underline{\mathbf{I}}_{\mathbf{t}}^{-\mathbf{1}}\left[\sum_{j=1}^{k} \mathbf{S}\left(\rho_{\mathbf{m}_{\mathbf{j}}}\right) \dot{m}_{j}\right]\left(\mathbf{R}_{\mathbf{B}_{\mathbf{R}} \mathbf{w}_{\mathbf{R}}} U+W\right) \\
& -2 \underline{\underline{\mathbf{I}}}_{\mathbf{t}}^{-\mathbf{1}} \sum_{j=1}^{k} m_{j}\left[\left(\rho_{m_{j}}^{T} \dot{\rho}_{m_{j}}\right) \mathbf{I}_{\mathbf{3} \times \mathbf{3}}-\dot{\rho}_{m_{j}} \rho_{m_{j}}^{T}\right]\left(\omega_{B_{R} B_{T}}+\mathbf{R}_{\mathbf{B}_{\mathbf{R}} \mathbf{B}_{\mathbf{T}}} \omega_{B_{T}}\right) \\
& \text { - } \underline{\underline{\mathbf{I}}}_{\mathbf{t}}^{-\mathbf{1}} \sum_{j=1}^{k} \dot{m}_{j}\left[\left(\rho_{m_{j}}^{T} \rho_{m_{j}}\right) \mathbf{I}_{\mathbf{3} \times \mathbf{3}}-\rho_{m_{j}} \rho_{m_{j}}^{T}\right]\left(\omega_{B_{R} B_{T}}+\mathbf{R}_{\mathbf{B}_{\mathbf{R}} \mathbf{B}_{\mathbf{T}}} \omega_{B_{T}}\right)
\end{aligned}
$$

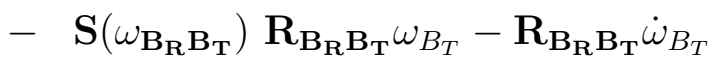

$$
\begin{aligned}
& -\underline{\mathbf{I}}^{-1} \dot{m} \mathbf{S}\left(\rho_{R}\right)\left(\mathbf{R}_{\mathbf{B}_{\mathbf{R}} \mathbf{B}_{\mathbf{T}}} \mathbf{R}_{\mathbf{B}_{\mathbf{T}} \mathbf{I}} \dot{r}_{B_{T}}+\mathbf{R}_{\mathbf{B}_{\mathbf{R}} \mathbf{B}_{\mathbf{T}}} V_{\dot{m}}\right)
\end{aligned}
$$

Note that the above set of equations, Eqs. (6.1) and (6.2) are not in the standard state-space form, which is the most convenient for numerical simulation. After some manipulation, these equations become

$$
\begin{aligned}
\dot{\mathcal{X}}_{R} & =\left(\mathbf{I}_{\mathbf{3} \times \mathbf{3}}-\mathbf{f}_{\mathbf{1}} \mathbf{f}_{\mathbf{2}}\right)^{-1}\left(\mathbf{f}_{\mathbf{1}} c_{2}+c_{1}\right), \\
\dot{\omega}_{B_{R} B_{T}} & =\mathbf{f}_{\mathbf{2}}\left(\mathbf{I}_{\mathbf{3} \times \mathbf{3}}-\mathbf{f}_{\mathbf{1}} \mathbf{f}_{\mathbf{2}}\right)^{-1}\left(\mathbf{f}_{\mathbf{1}} c_{2}+c_{1}\right)+c_{2}
\end{aligned}
$$

which are the dynamics equations written in the state-space form and to be used in computer simulations. 


\section{CHAPTER 7}

\section{MODELING THE VORTEX AND ITS EFFECT}

It is to be noted that the wind effect terms constituting the elements $W, \dot{W}$ in the receiver's equations of motion derived earlier are considered to be based on the uniform wind distribution acting at the receiver's CM, expressed in its body frame. But, the vortex-induced wind field acting on the receiver aircraft is non-uniform in nature. Therefore, to be able to use the aircraft equations of motion without doing any modifications, there is a need to approximate the non-uniform induced wind components and gradients by equivalent uniform wind and gradients. Once a fairly reasonable approximation can be achieved, the implementation of aerodynamic coupling between the tanker and the receiver becomes far more direct and computationally efficient than the conventional procedure which involves first the calculation of induced forces and moments from the wind distribution, and then inserting these forces and moments in the aircraft dynamics equations.

In the dynamic model of this thesis for aerial refueling, the tanker is represented by two sets of vortices each consisting of one bound and two tip (trailing) vortices, with one set for the wing and the other for the horizontal tail. These six vortex filaments induce additional wind velocities on the body of the receiver aircraft, which, in turn, cause changes in the forces and moments experienced by the receiver. However, instead of attempting to estimate the induced forces and moments on the follower, the induced wind velocities and wind gradients are computed. The induced wind velocities are written as a function of the relative separation as well as the relative orientation between the tanker and the receiver using a modified horseshoe vortex model based on the Helmholtz profile. 
Since the induced wind and wind gradients are non-uniform along the body dimensions of the receiver aircraft, an averaging technique is implemented to compute the effective wind and wind gradient as uniform approximations. The effective wind components and gradients are introduced into the nonlinear aircraft equations that include the components of wind and the temporal variation of wind in the body frame to determine the effect on the receiver's dynamics. The effect of vortex decay over time is also included in our model. Special care has been taken to accommodate different geometrical dimensions for the tanker and the receiver aircraft and also to include many useful geometrical parameters of the aircraft like the wing sweep angle, the dihedral angle and the relative distance between the CM of the UAV and the aerodynamic center of the wing, in estimating the vortex-effect experienced during aerial refueling. For further details of the actual vortex model and the averaging technique used to estimate the vortex-effect on the receiver, see Ref.[58]. Note that bound vortices were not considered in this reference, but its overall approach is followed herein. 


\section{CHAPTER 8}

\section{APPLICATION}

This section explains how the physical parameters of the receiver aircraft and its fuel tanks are incorporated as mathematical quantities that are included in the derivation of the equations of motion. It further presents some results of the simulation that incorporates the equations of motion developed herein as the model of the receiver aircraft dynamics during aerial refueling. The simulation also includes the full 6-DOF nonlinear dynamics of the tanker aircraft. In the simulation, the position and orientation of the receiver relative to the tanker are controlled by a gain-scheduling linear controller while the tanker flies in a racetrack maneuver. The simulation contains the ICE (Innovative Control Effectors) unmanned aircraft as the receiver being refueled from a KC-135 tanker aircraft. For details of the tanker or receiver being simulated or the controller being employed, see Refs. [28] or [59].

\subsection{Fuel Tank Configuration}

First, the overall fuel flow rate $(\dot{m})$, which may be time-varying, from the tanker to the receiver is considered to be specified as an external input. Further, the part of the total fuel flow that is sent to each individual fuel tank is specified. For example, Fig. 8.1 shows the sketch of the ICE aircraft containing four fuel tanks in it. In normal circumstances, the refueling begins with the forward tanks with the total fuel flow distribution being such that

$$
\dot{m}_{1}=\dot{m}_{2}=0.5 \dot{m}
$$




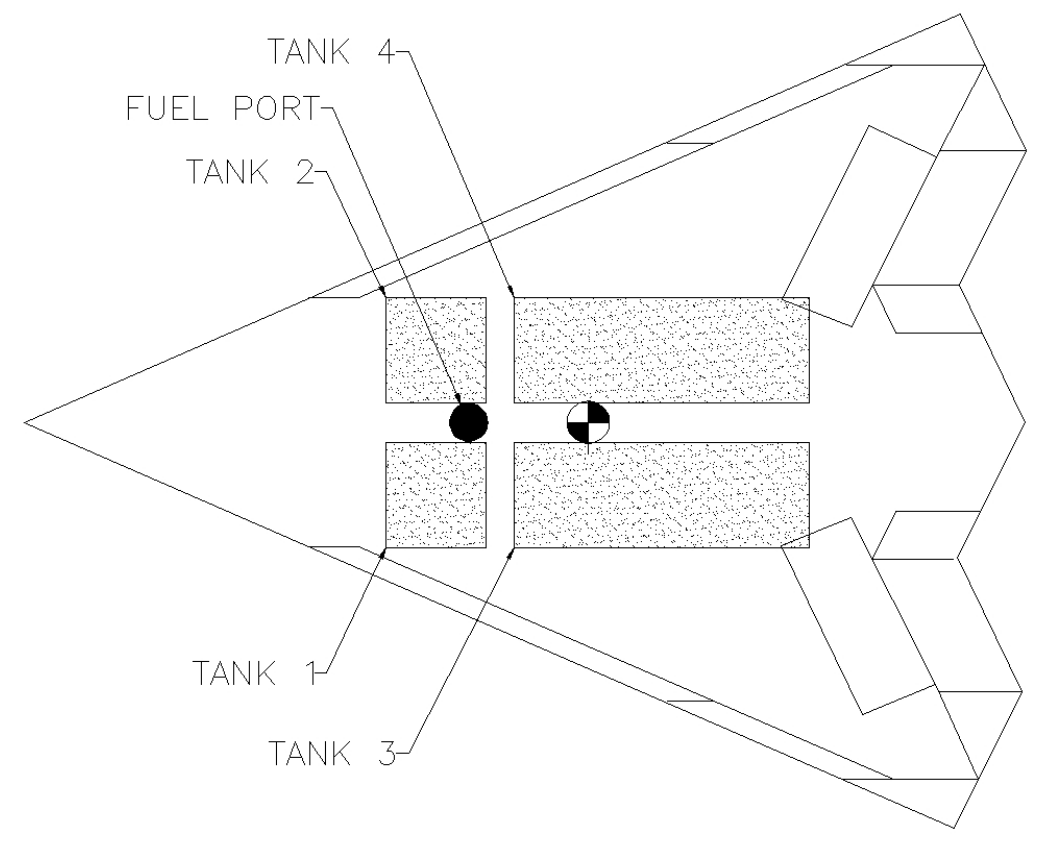

Figure 8.1. Receiver aircraft with its fuel tanks.

where the combined fuel capacity of tanks 1 and 2 totals $35 \%$ of the total aircraft fuel. After tanks 1 and 2 are full, the aft tanks begin refueling such that the total fuel flow distribution is described as

$$
\dot{m}_{3}=\dot{m}_{4}=0.5 \dot{m}
$$

where the combined fuel capacity of tanks 3 and 4 totals $65 \%$ of the total aircraft fuel. The actual mass contained in the $j^{\text {th }}$ fuel tank at any instant of time can be computed using the formula

$$
m_{j}(t)=\int_{0}^{t} \dot{m}_{j}(\tau) \mathrm{d} \tau
$$

where $t$ represents the time elapsed since the refueling operation had begun. If there is any residual fuel in the tank before the start of refueling, that can be considered as part of the receiver aircraft. Therefore, without any loss of generality, it is assumed that $m_{j}(0)=0$. 
The next variable to be determined is $\underline{\rho}_{m_{j}}$ which is the position vector of the fuel mass concentrated at the $j^{\text {th }}$ point, expressed in $B_{R^{-}}$frame. Recall the assumption that fuel in each fuel tank is concentrated at its CM. In general, the initial location $\underline{\rho}_{m_{j}}(0)$ is considered to be the mid-point of the base of the $j^{\text {th }}$ fuel tank or the surface of the remaining fuel (Fig. 8.2). As the fuel flows into the fuel tank, the vector $\underline{\rho}_{m_{j}}(t)$ should always point at the CM of the fuel (Fig. 8.3).

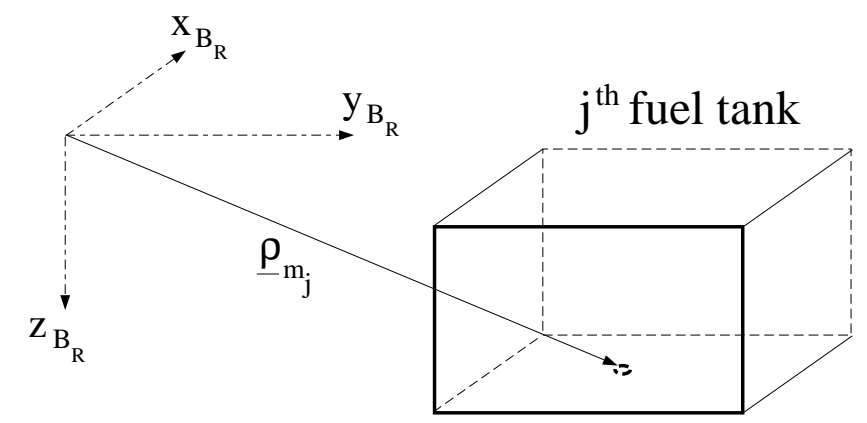

Figure 8.2. Initial condition for lumped mass position vector.

For example, consider the two-view diagram of fuel tank 1 as shown in Fig. 8.3. Initially,

$$
\underline{\rho}_{m_{1}}(0)=\left[\underline{\hat{B}}_{R}\right]^{T}\left[\begin{array}{c}
x_{1}(0) \\
y_{1}(0) \\
z_{1}(0)
\end{array}\right]
$$

During the refueling, $x_{1}(t)$ and $y_{1}(t)$ will be constants and equal to their initial values. This follows from the assumptions that the fuel tanks are rectangular and the fuel stays level within each tank. However, since the level of the fuel is rising, $z_{1}(t)$ will be timevarying. From Eq. (8.1), if $\dot{m}_{1}(t)$ is the fuel flow rate into tank 1, the amount of fuel mass in the tank increases as

$$
m_{1}(t)=\int_{0}^{t} \dot{m}_{1}(\tau) \mathrm{d} \tau
$$




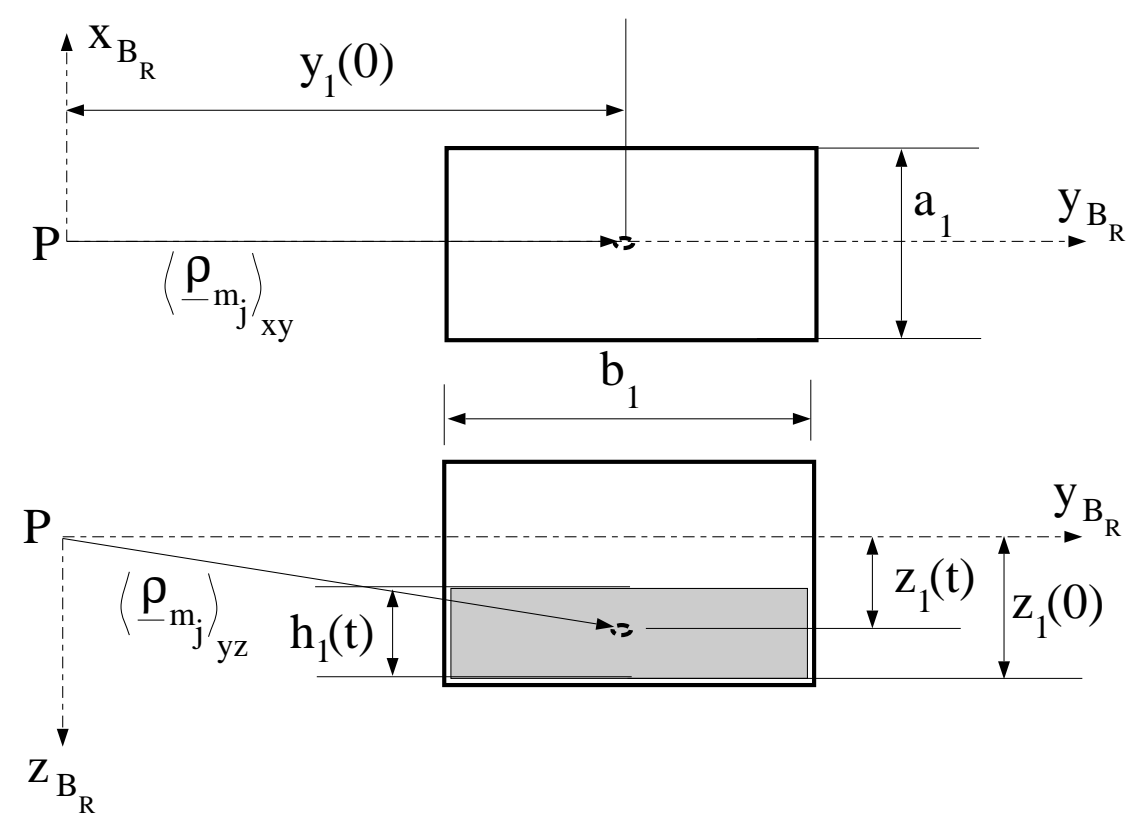

Figure 8.3. Two-view diagram of fuel tank 1.

With the assumptions stated above, while fuel is flowing in, the height of the CM of the fuel from the base of the tank can be expressed as

$$
h_{1}(t)=\frac{m_{1}(t)}{\rho_{\text {fuel }} a_{1} b_{1}}
$$

where $a_{1}$ and $b_{1}$ denote the length and breadth of fuel tank 1 (Fig. 8.3), and $\rho_{\text {fuel }}$ is the density of the fuel being transferred. Thus, the time-varying position vector of fuel tank 1 is obtained as

$$
\underline{\rho}_{m_{1}}(t)=\left[\underline{\hat{B}}_{R}\right]^{T}\left[\begin{array}{c}
x_{1}(0) \\
y_{1}(0) \\
z_{1}(0)-\frac{h_{1}(t)}{2}
\end{array}\right]
$$

The $\underline{\rho}$-vector as a function of time for the other fuel tanks can be formulated similarly. Then, the position vectors along with their time derivatives for all the fuel tanks are used in the receiver's equations of motion. 


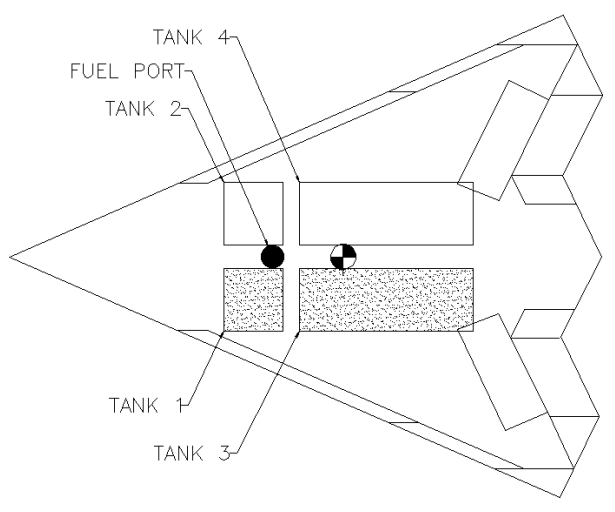

(a)

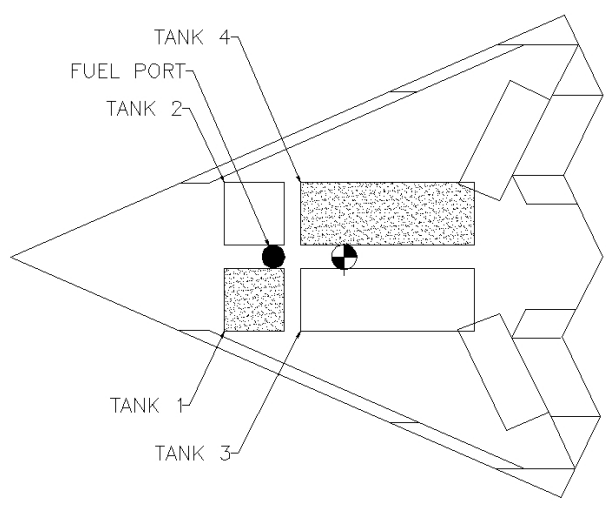

(b)

Figure 8.4. Receiver fuel tank failure cases: (a) Right-hand tanks do not fill and (b) Right-hand forward and left-hand aft tanks do not fill.

\subsection{Simulation Results}

In the simulation, the tanker aircraft flies at the nominal altitude of 7010 meters at a speed of $190 \mathrm{~m} / \mathrm{s}$ in a racetrack maneuver. However, note that this is the nominal flight condition and that small deviations from the nominal conditions occur as the tanker transitions between the legs of the racetrack maneuver. While the tanker flies in the racetrack manuever, the receiver aircraft needs to maintain the contact position of $(-25.33,0,6.46)$ meters relative to the body frame of the tanker. Maintaining the contact position enables the tanker's refueling boom to deliver 0.04416 cubic meters of JP-4 fuel per second $(700 \mathrm{gal} / \mathrm{min})$ at the speed of $5.45 \mathrm{~m} / \mathrm{s}$. It is assumed that the refueling boom is situated at an incline of 30 degrees downward from the negative x-axis of the tanker's body frame during the fuel transfer. The following is a presentation of the results from the simulation of refueling while the tanker is at (i) straight-level flight and (ii) constantaltitude, constant-speed turn. The configuration of fuel tanks in the receiver aircraft and the scheduling of the tanks are given in the previous section.

Figures 8.5 through 8.10 depict the results of the simulations when executed for a straight level flight condition for three different cases, as labeled in the figure legends. 
Case 1 is a normal refuel scenario where the refueling begins at 25 seconds. In Case 1 , both forward tanks fill first, each receiving half of the maximum tanker boom fuel flow rate until filled to their commanded capacity, after which both aft fuel tanks receive half of the maximum tanker boom fuel flow rate until filled to their commanded capacity. Case 2 is a refuel failure scenario where neither of the right-hand fuel tanks (labeled Tank 2 and Tank 4 in Fig. 8.1) receive fuel, but the left-hand forward tank still fills first, followed by the left-hand aft; this failure scenario is depicted in Fig. 8.4(a). Case 3 is a refuel failure scenario where the right-hand forward and left-hand aft tanks do not fill, which is depicted in Fig. 8.4(b). It should be noted that, in order to allow for more accurate comparisons of the normal case to the failure cases, the fuel tanks in the normal case are commanded to fill only to half of their capacities so that the transition from refueling the forward tanks to the aft tanks occurs at the same time in all three cases. This measure additionally ensures that the post-refuel total aircraft weight for the normal case matches that for the failure cases.

To be able to maintain the nominal refueling contact position, the deviation from the refueling position as well as the deviation in relative orientation should be minimal. To analyze the performance of the aircraft in terms of these important requirements, phase portraits of position and orientation are presented in Figs. 8.5 and 8.6, respectively. Note that the y-deviation extends up to 0.3 and -0.2 meters with Case 3 resulting in deviations significantly greater than those of the other cases. It is interesting to note that, while Cases 2 and 3 have, as expected, the same $\mathrm{y}$-deviations in the negative $\mathrm{x}$ - and y-quadrant while Tank 1 is filled, their $\mathrm{y}$-deviations in the positive $\mathrm{x}$ - and $\mathrm{y}$-quadrant are in opposite directions due to the fact that opposite-side aft tanks are being filled in these cases (Tank 3 in Case 2 and Tank 4 in Case 3). The x-deviation is between -0.2 and 0.2 meters in all three cases and z-deviation is between -0.1 and 0.15 meters. The deviations 

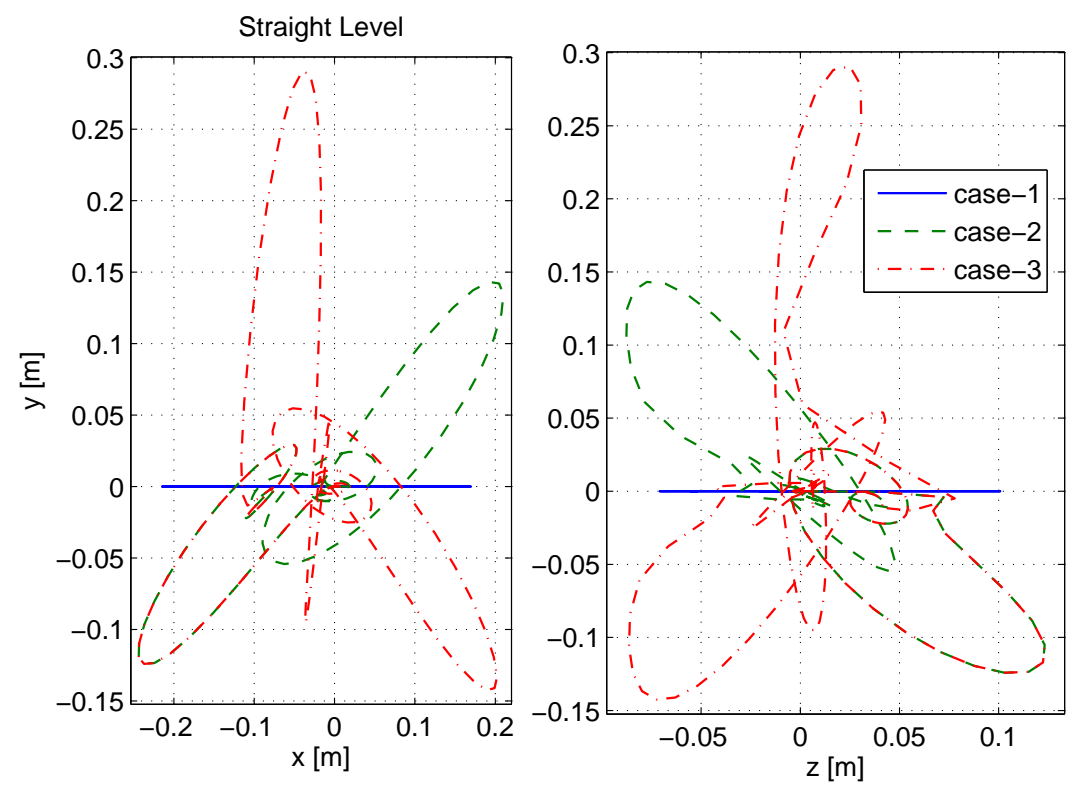

Figure 8.5. Deviation of the receiver position from the refueling position in straight level flight.

are smallest for Case 1, which results in zero y-deviation as expected based on the fuel tank symmetry.

Figure 8.6 shows the phase portraits of Euler angles to illustrate the deviation of the receiver orientation from the tanker orientation. The pitch angle variation is approximately the same (below $1.5 \mathrm{deg}$ ) for all cases while Case 3 causes a greater range of deviation of both yaw and bank angles. As with the y-deviation of Fig. 8.5, it is interesting to note the portion of the Case 3 yaw deviation which is opposite of the Case 2 yaw deviation due to the asymmetric tank refueling.

Fig. 8.7 illustrates the $\mathrm{x}^{-}, \mathrm{y}$ - and $\mathrm{z}-$ components of the receiver's position relative to the tanker in the time domain. Correlations can be drawn between Figs. 8.7 and 8.5, such as the magnitude of the y-position spikes resulting from the Case 3 scenario. The first such spike at 25 seconds is the same for both Cases 2 and 3 due to the fact that both cases begin by refueling the left-hand forward tank, but Case 3 second spike around 70 

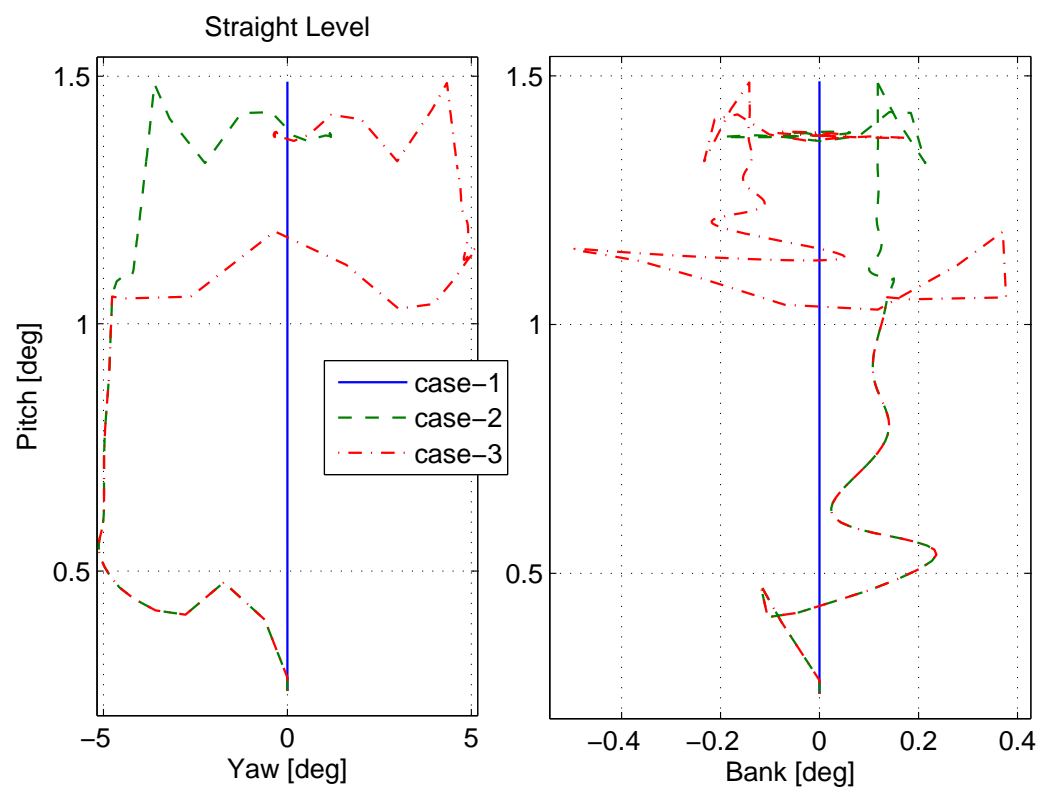

Figure 8.6. Receiver deviation in relative orientation in straight level flight.

seconds is the result of the cessation of Tank 1 refueling and the commencement of (righthand aft) Tank 4 refueling, and it is this second larger spike which correlates to the largest y-deviation depicted in the phase portrait. Similarly, the opposite-direction y-position spikes at about 140 seconds are the result of refuel completion and the reorientation of the aircraft into an appropriate trim condition after being refueled in opposite-side aft tanks, which correlates to the opposite-direction y-deviation spikes seen in the phase portrait. This behavior is mathematically represented by the third term of Eq. (5.27), which illustrate the contribution of the individual fuel tank masses and their derivatives, along with the fuel CM locations and their derivatives, to the receiver's translational dynamics.

Fig. 8.8 shows how the orientation of the receiver changes in terms of Euler angles relative to the tanker's body frame in the time domain during straight level flight in the three cases. As in the case with the position plots, correlations can be drawn between Figs. 8.8 and 8.6, such as the opposite-direction tendencies of the yaw and bank angles 

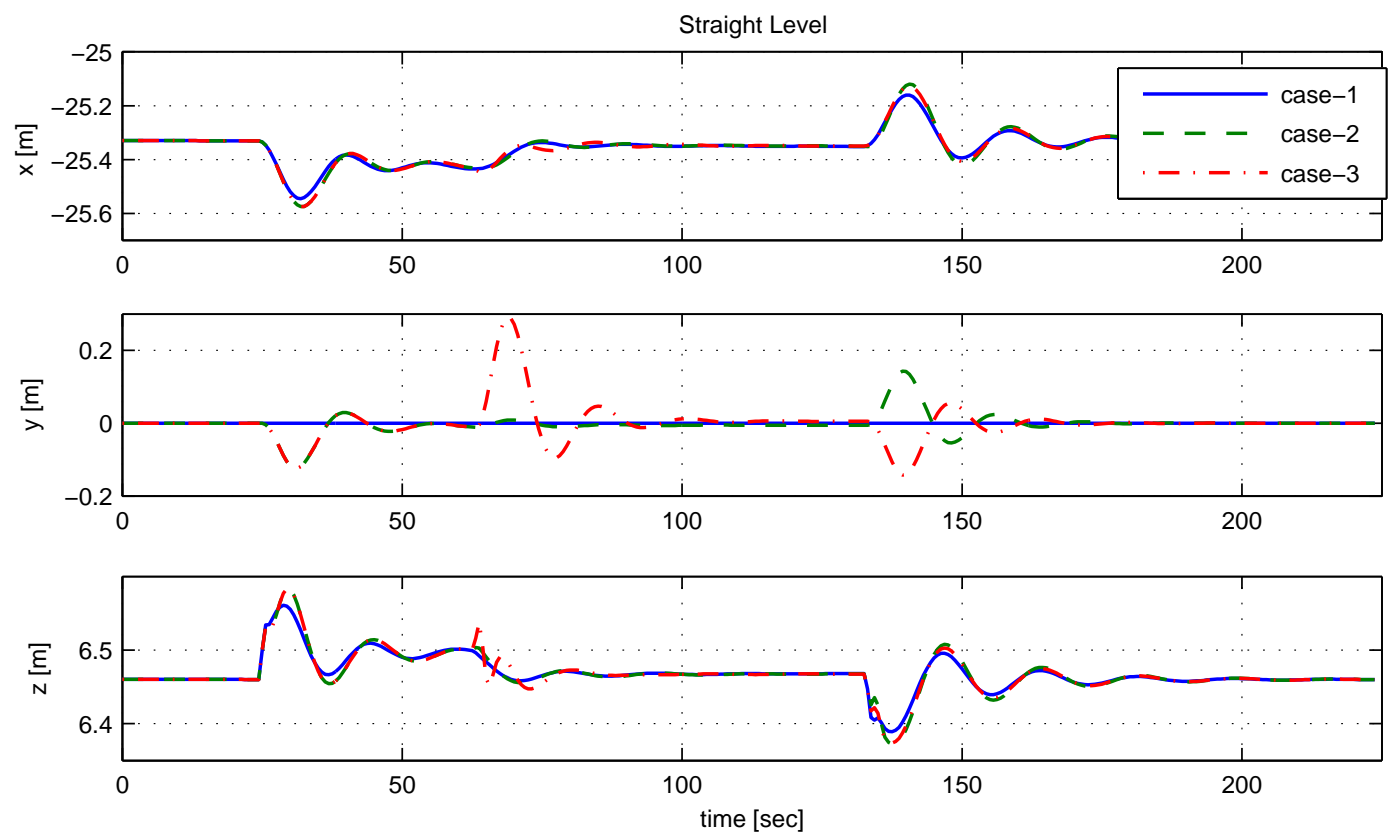

Figure 8.7. Time history of the deviation of the receiver position from the refueling position in straight level flight.

immediately following the Case 3 transition from the forward to diagonal aft fuel tank when compared to the same-side forward to aft tank transition of Case 2. These moments are largely due to the contribution of the second, third, fourth, fifth, sixth, and eighth terms of Eq. (5.63), which express the relationship between the derivatives of the individual fuel tank masses and center-of-gravity locations to the receiver's rotational dynamics. It is also interesting to note that, after refuel completion, the yaw trim angle is different for Cases 2 and 3, where the Case 2 results in a slightly positive yaw trim angle and Case 3 results in a very slightly negative yaw trim angle. Note also that there is, albeit very small, a nonzero steady-state bank angle in Cases 2 and 3. This demonstrates the effect of the asymmetric mass distribution on the steady-state trim condition.

Figures 8.9 and 8.10 illustrate the values of the control variables in all three cases of straight level flight. Figure 8.9 illustrates the deflections of the three control effectors: elevon, pitch flap, and clamshell. In all three cases, very small deflections (compared 

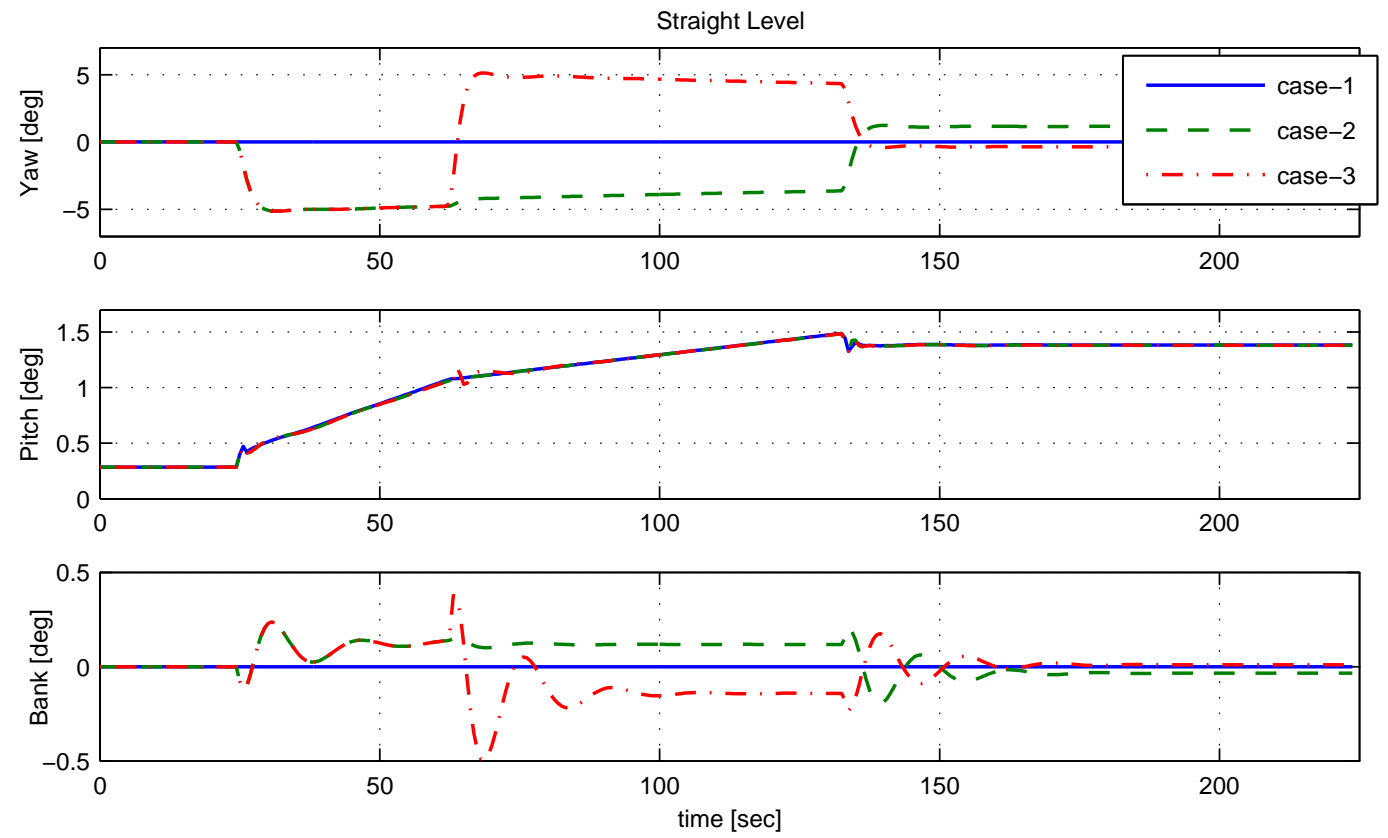

Figure 8.8. Time history of the receiver relative orientation deviation in straight level flight.

to the saturation deflections) in all the three effectors are used to maintain the refuel contact position throughout the simulation. Both the elevon and clamshell deflections can be compared with the roll and yaw angle histories in Fig. 8.8 to show how these effectors reacted to the refueling in the failure cases and how their steady-state positions corresponded to the receiver's steady-state Euler angles. It can be seen that, when the refueling of Case 3 transitioned from the forward to the diagonal aft tank, both the elevon and clamshell reacted immediately to compensate for the opposite-side tank refueling, and it is interesting to note how the elevon and pitch flap angle magnitudes change steadily throughout the refueling to account for the steadily growing fuel tank masses. This phenomenon is accounted for by the contribution of the gravitational moment caused by the fuel masses as presented in Eq. (4.11) to the total moment of the external forces about the origin of $B_{R}$-frame as presented in Eq. (4.10). Figure 8.10 illustrates the level 

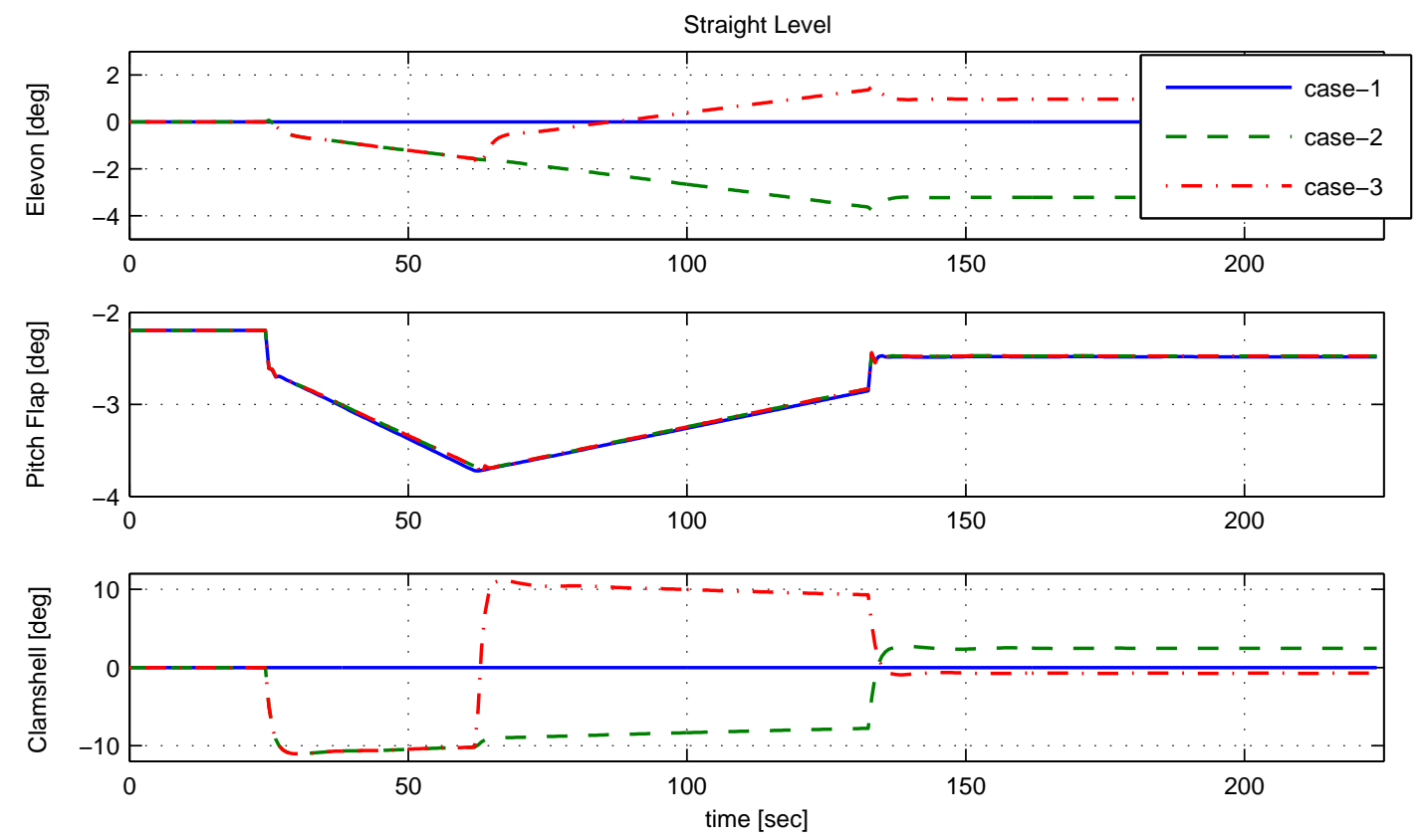

Figure 8.9. Time history of receiver control surface deflections in straight level flight.

of throttle in the three cases, which prove to be very similar. As expected, the throttle tends to grow as the refueling occurs (beginning at 25 seconds) to keep the receiver at the commanded velocity while experiencing mass increase, and the steady-state throttle value is somewhat higher than initially due to the higher post-refuel aircraft weight.

Figures 8.11 through 8.16 depict the results of the simulation when executed for a U-turn maneuver scenario which involves the tanker beginning to turn 25 seconds into the simulation with a specified yaw rate until the yaw angle change reaches 180 degrees. The commanded yaw rate for the tanker in a racetrack maneuver is generated from a 1.7 $\mathrm{deg} / \mathrm{sec}$ step response of fourth order linear filter with time constants of 10, 10, 10 and 1 seconds. In these figures, Case 1 involves the turn maneuver only with no refueling, included as a reference to determine how much of the receiver's response is due solely to the refueling. Case 2 is the same normal refuel scenario introduced in the straight level flight case, with refueling beginning with the forward tanks at 25 seconds and continuing 


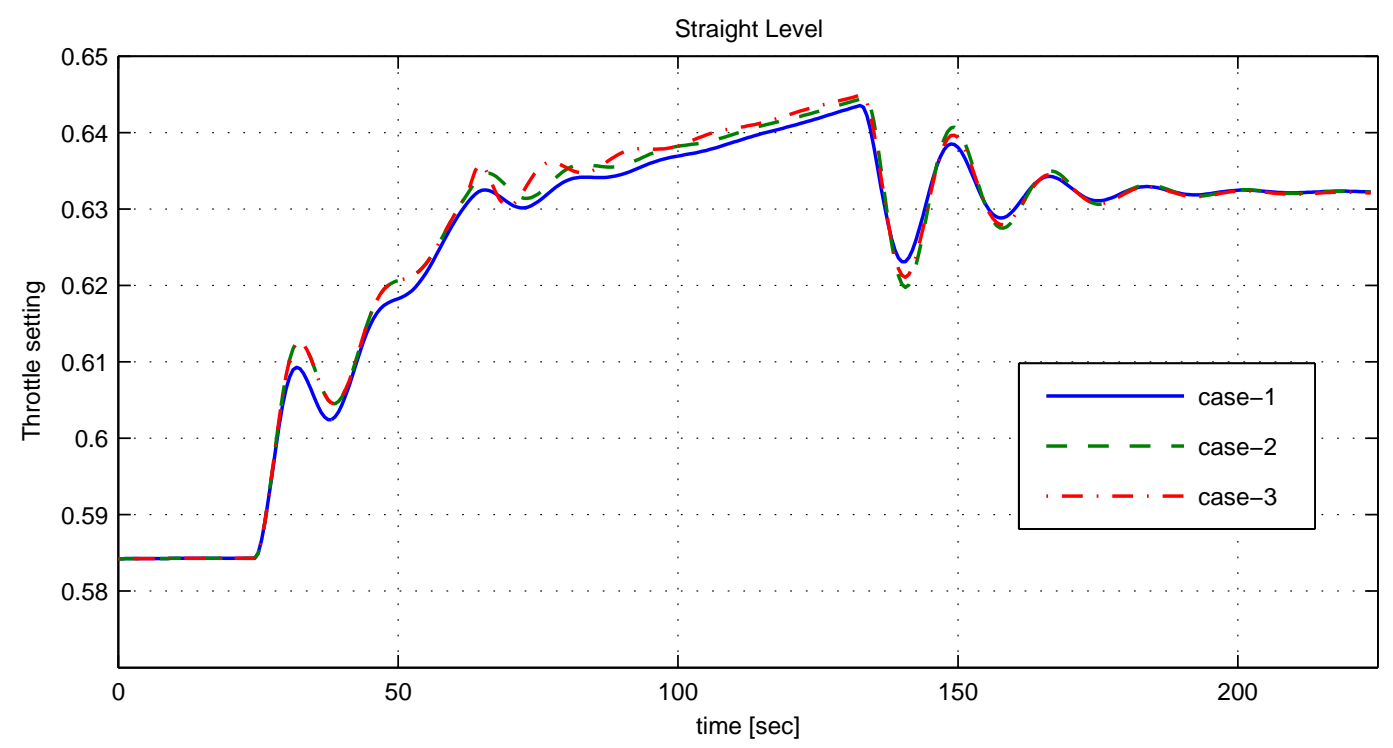

Figure 8.10. Time history of receiver throttle setting in straight level flight.

with the aft tanks after the forward tanks are filled to commanded capacity. Case 3 is the refuel failure scenario where the right-hand forward and left-hand aft tanks do not fill, which is depicted in Fig. 8.4(b). Once again, the fuel tanks in the normal case are commanded to fill only to half of their capacities so that the transition from refueling the forward tanks to the aft tanks occurs at the same time in Cases 2 and 3 while ensuring that the post-refuel total aircraft weight for the normal case matches that for the failure case.

As in the straight level flight case, the deviation from the refueling position as well as the deviation in relative orientation should be minimal. To analyze the performance of the aircraft in terms of these important requirements, phase portraits of position and orientation are presented in Figs. 8.11 and 8.12, respectively. When compared with deviations in the straight level cases, Case 1 herein shows that the additional deviations are mostly due to the response of the receiver to the tanker turn. However, Figs. 8.11 and 8.12 show the additional deviations due to the symmetric and asymmetric fuel transfer. 

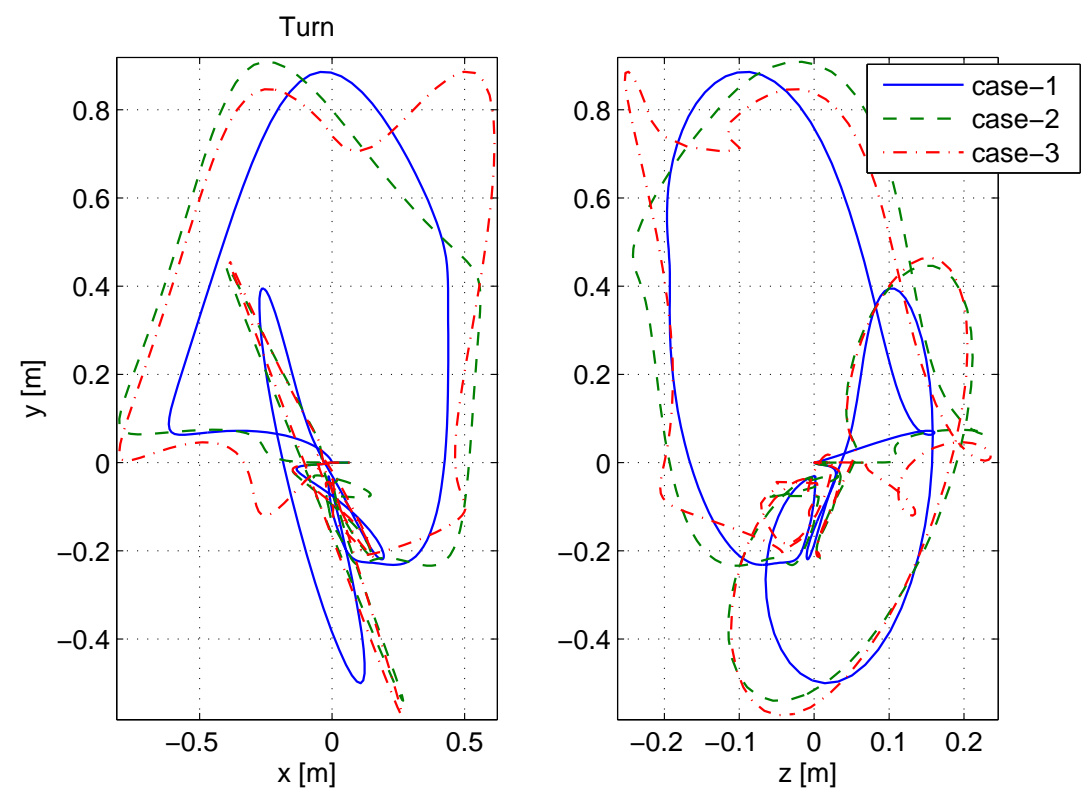

Figure 8.11. Deviation of the receiver position from the refueling position during turn.

The effects of the refueling are somewhat more pronounced in the Euler angle phase portrait of Fig. 8.12, where both refueling cases, especially the failure of Case 3 about the yaw axis, create orientation deviations significantly in excess of the orientation deviations due only to the turn.

Figure 8.13 illustrates the $\mathrm{x}^{-}, \mathrm{y}$ - and $\mathrm{z}^{-}$components of the receiver's position relative to the tanker in the time domain. Once again, correlations can be drawn between Figs. 8.13 and 8.11, such as the magnitude of the y-position displacement around 45 seconds resulting from the Case 3 forward-to-aft tank transition. Fig. 8.14 presents results comparable to the Euler angle phase portrait, where the yaw deviations due to Case 3 and the fuel tank transition thereof are quite apparent. It is also apparent in this plot that, when compared to the no-refueling Case 1, the steady-state pitch angle of Cases 2 and 3 is approximately one degree higher to account for the increased aircraft weight at the end of the refueling exercise. 

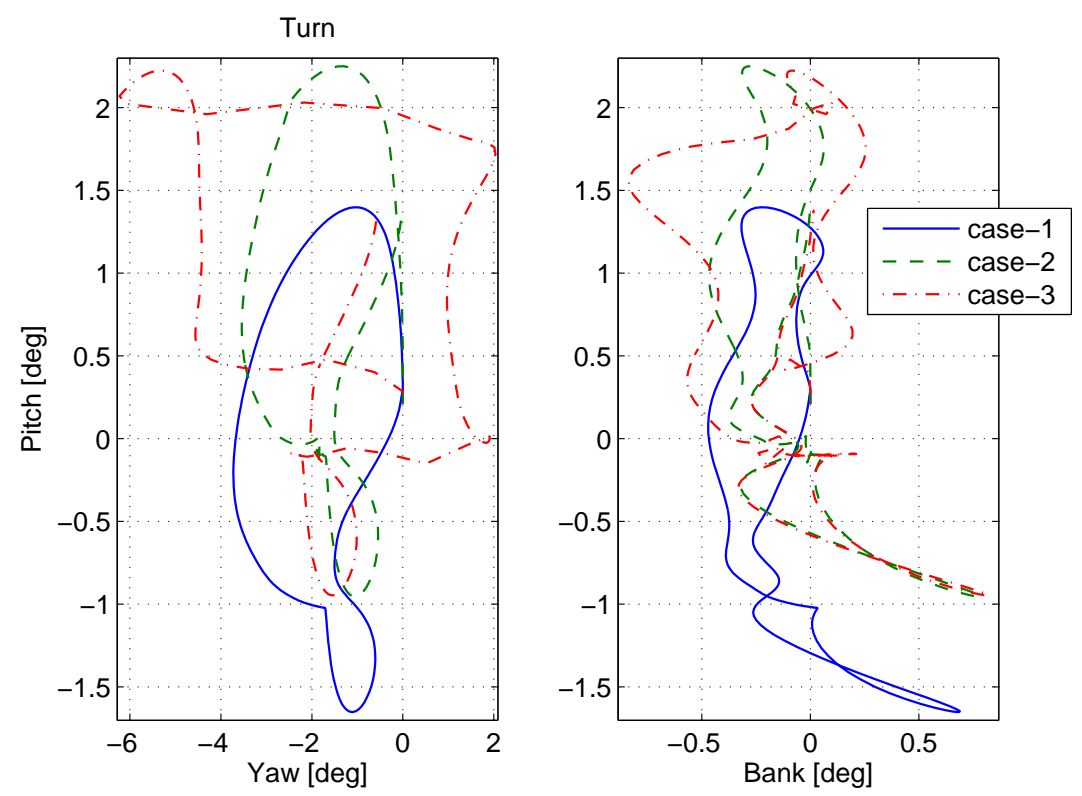

Figure 8.12. Receiver deviation in relative orientation during turn.
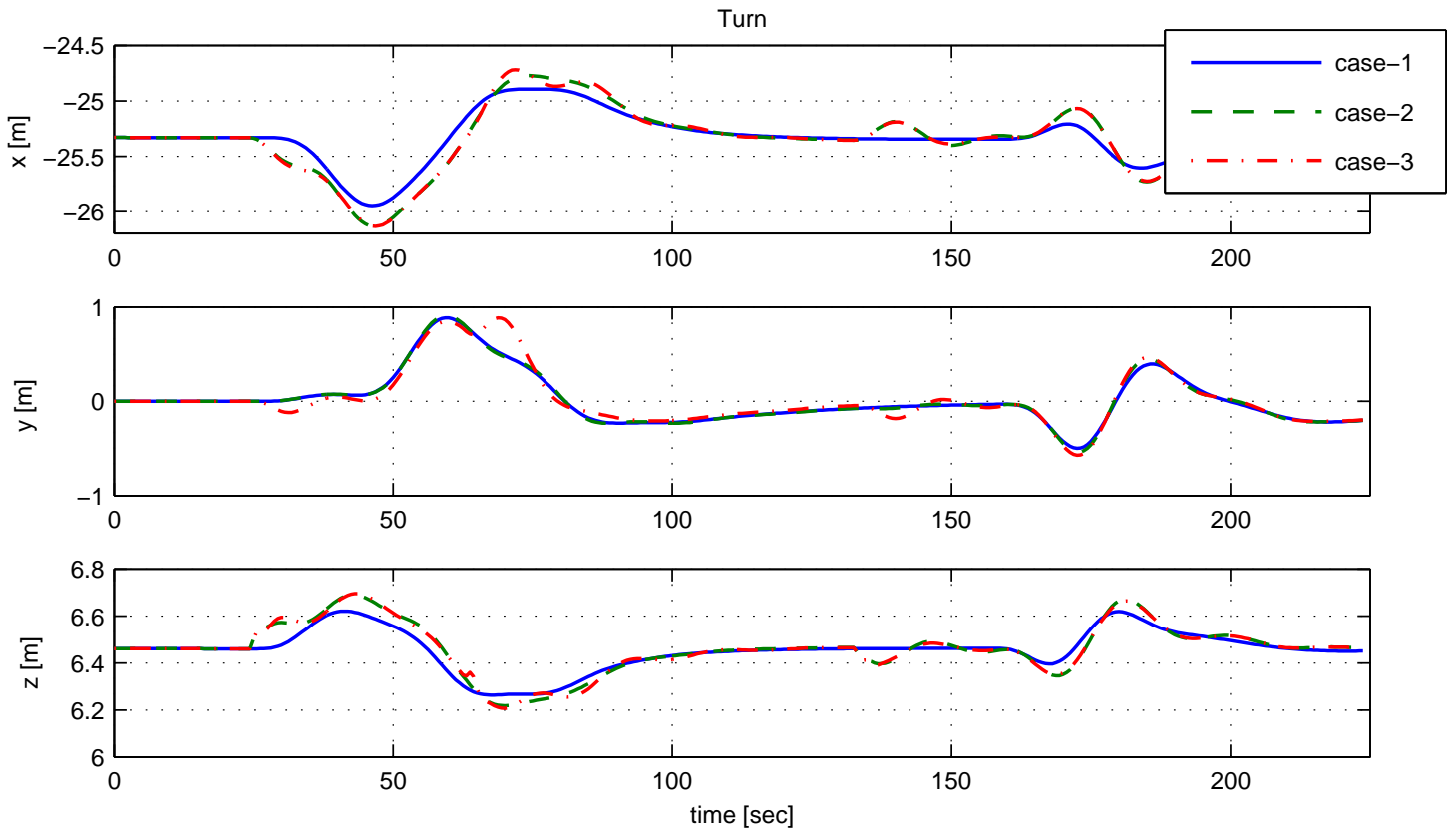

Figure 8.13. Time history of the deviation of the receiver position from the refueling position during turn. 

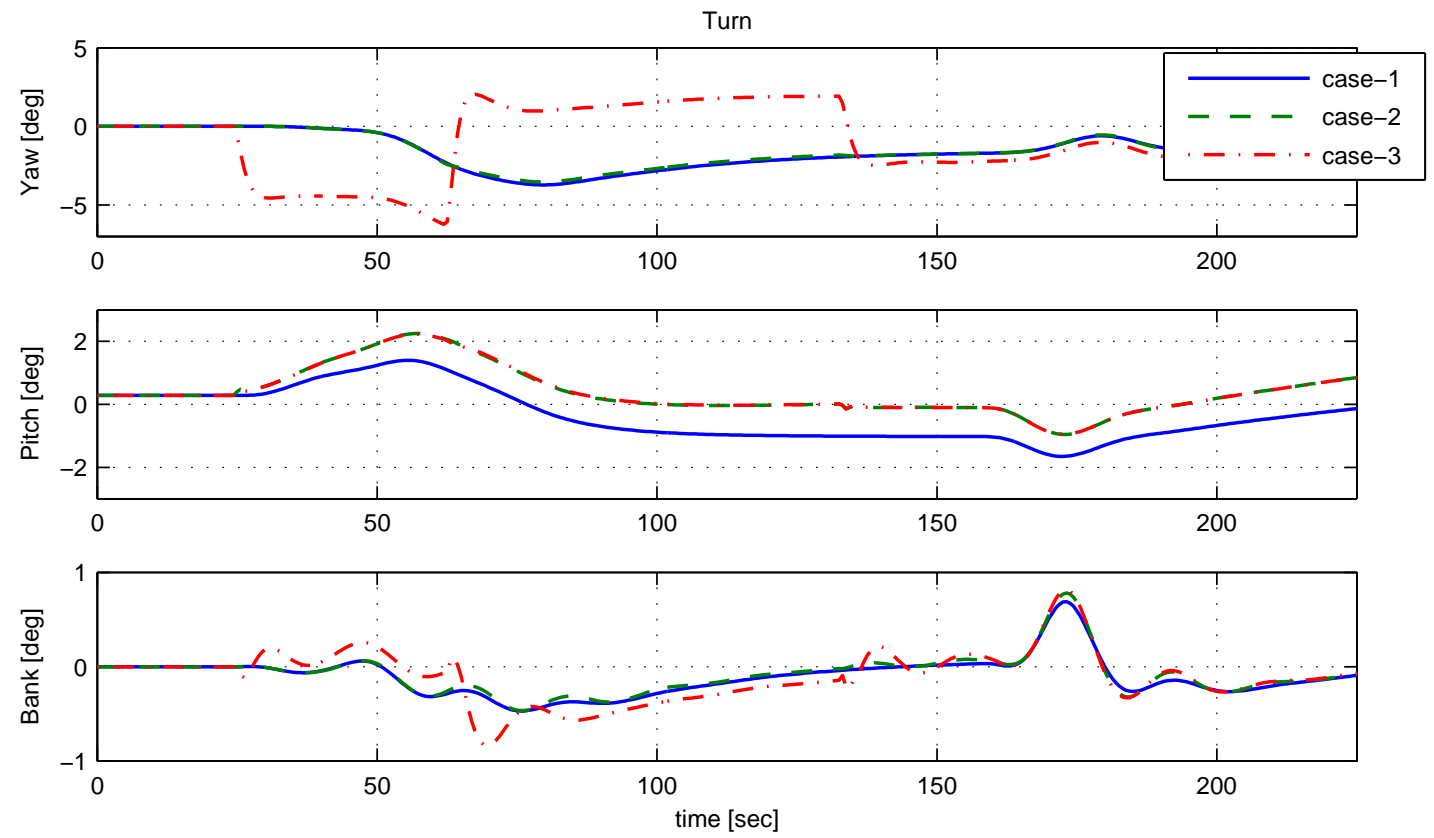

Figure 8.14. Time history of the receiver relative orientation deviation during turn.

Figures 8.15 and 8.16 illustrate the magnitudes of the control variables in all three turn maneuver cases. Figure 8.15 illustrates the deflections of the three control effectors and it is obvious that the tenancies of especially the elevon and clamshell follow those of the straight level flight case presented in Fig. 8.9. The differences between the effector deflections required for turning with and without refueling are also illustrated here, with the steady-state trim deflections being somewhat different both with and without fuel, and with and without asymmetric mass distribution. Figure 8.16 illustrates the level of throttle in the three cases, with Cases 2 and 3 having almost identical throttle requirements. Note that the throttle setting is higher in Cases 2 and 3 than in Case 1 during the turn as well as during steady-state after the turn and refueling are both completed. This is due to the mass transfer during the refueling and due to the higher post-refuel aircraft weight in steady-state. 

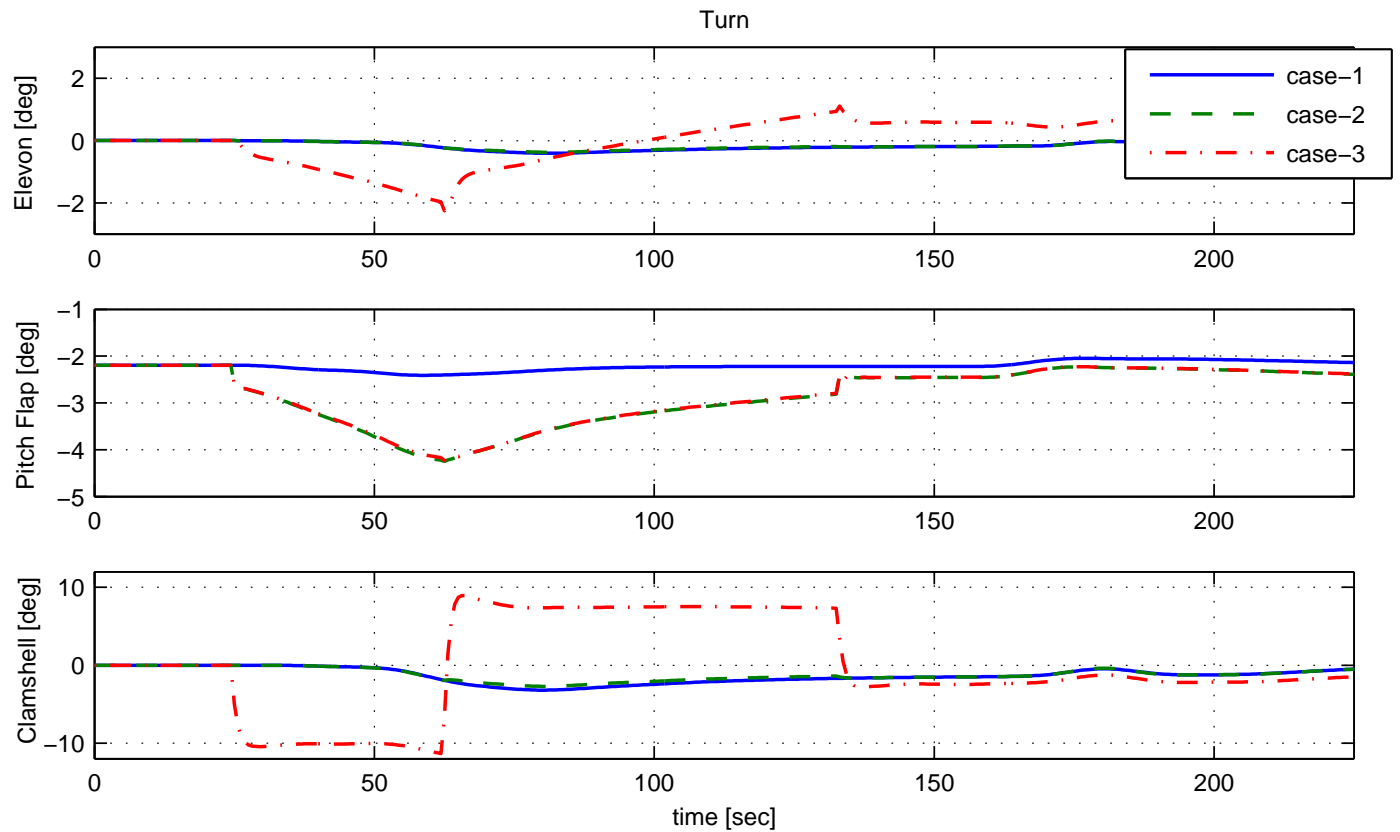

Figure 8.15. Time history of receiver control surface deflections during turn.

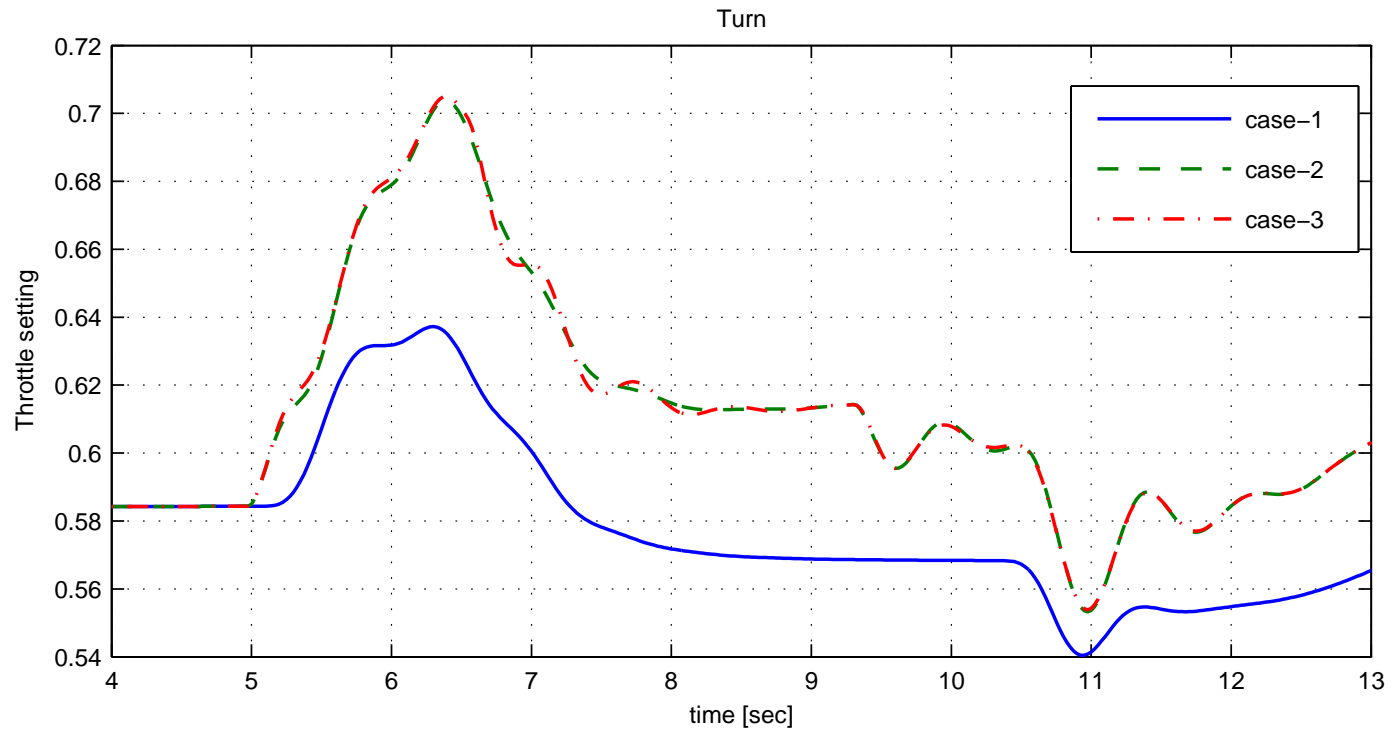

Figure 8.16. Time history of receiver throttle setting during turn. 

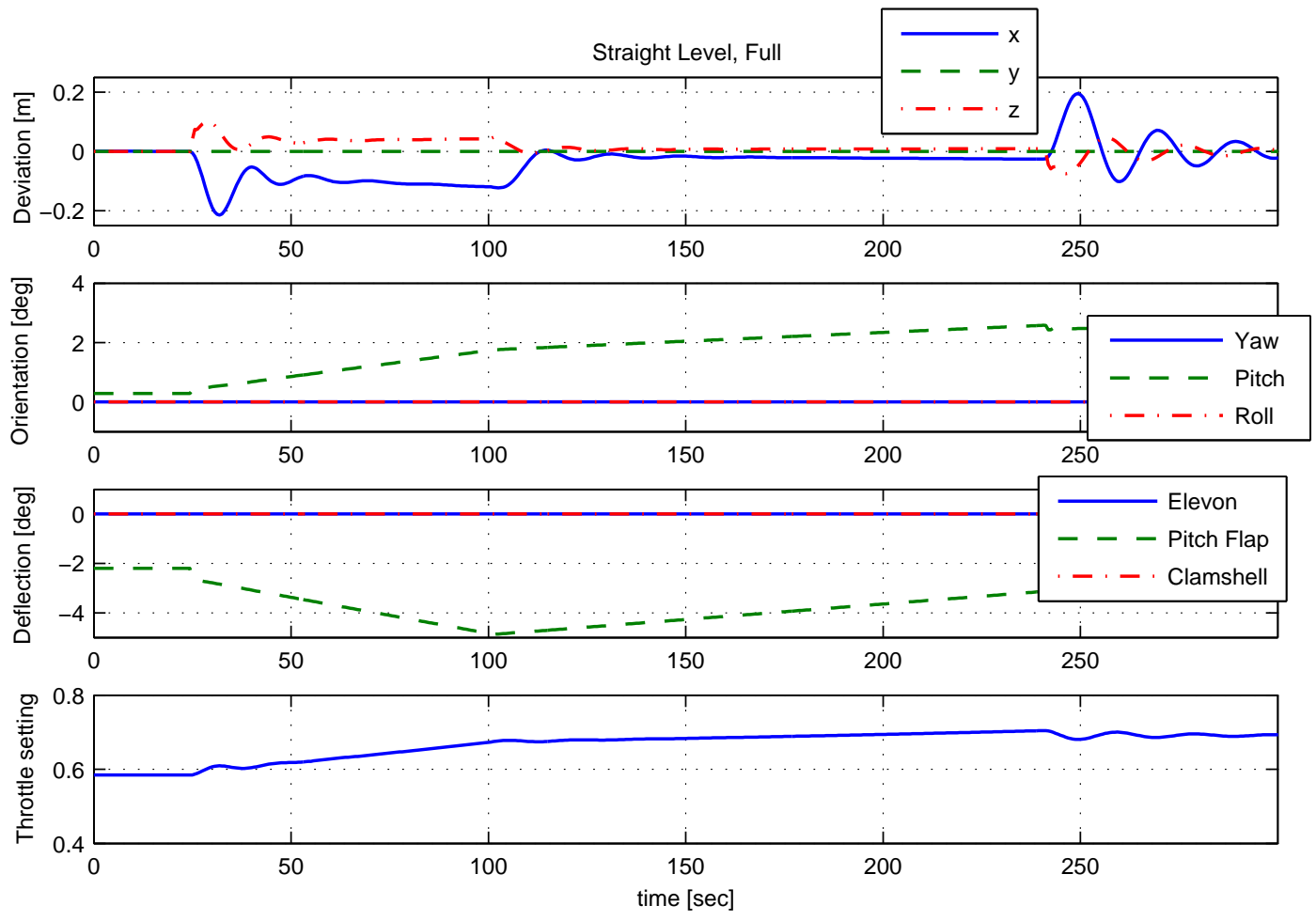

Figure 8.17. Time history of receiver in straight level flight becoming fully refueled.
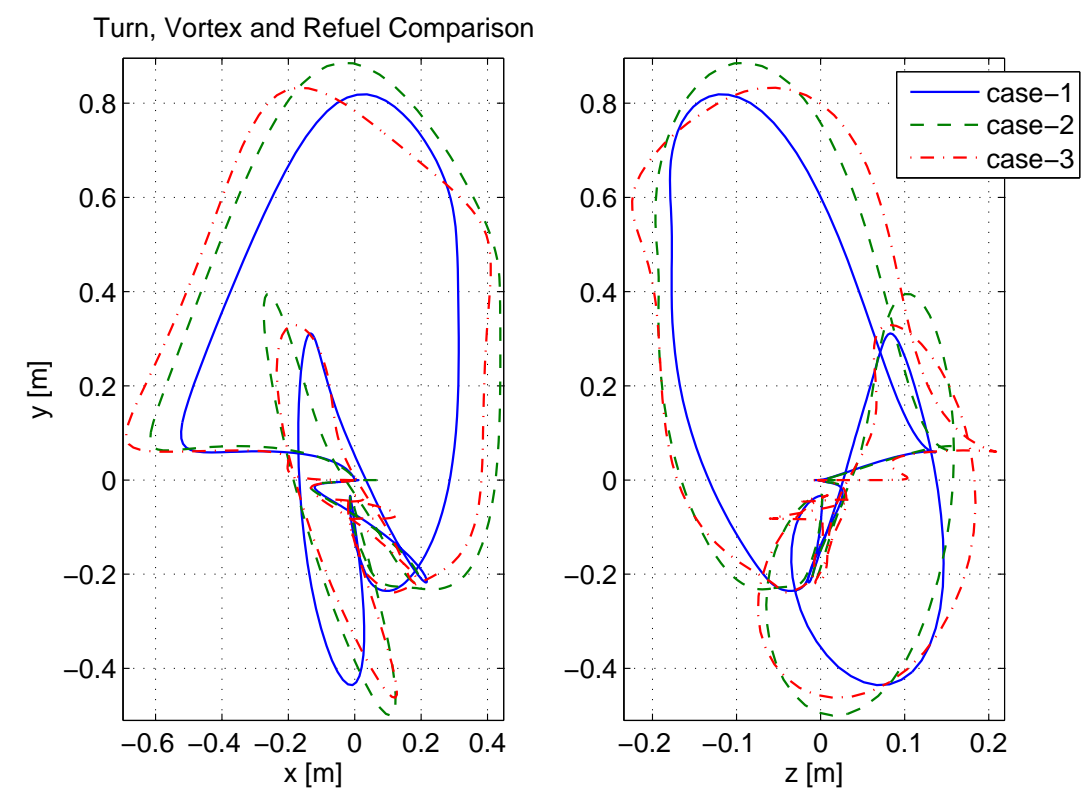

Figure 8.18. Deviation of the receiver position from the refueling position during turn for observation of vortex and refueling effects. 

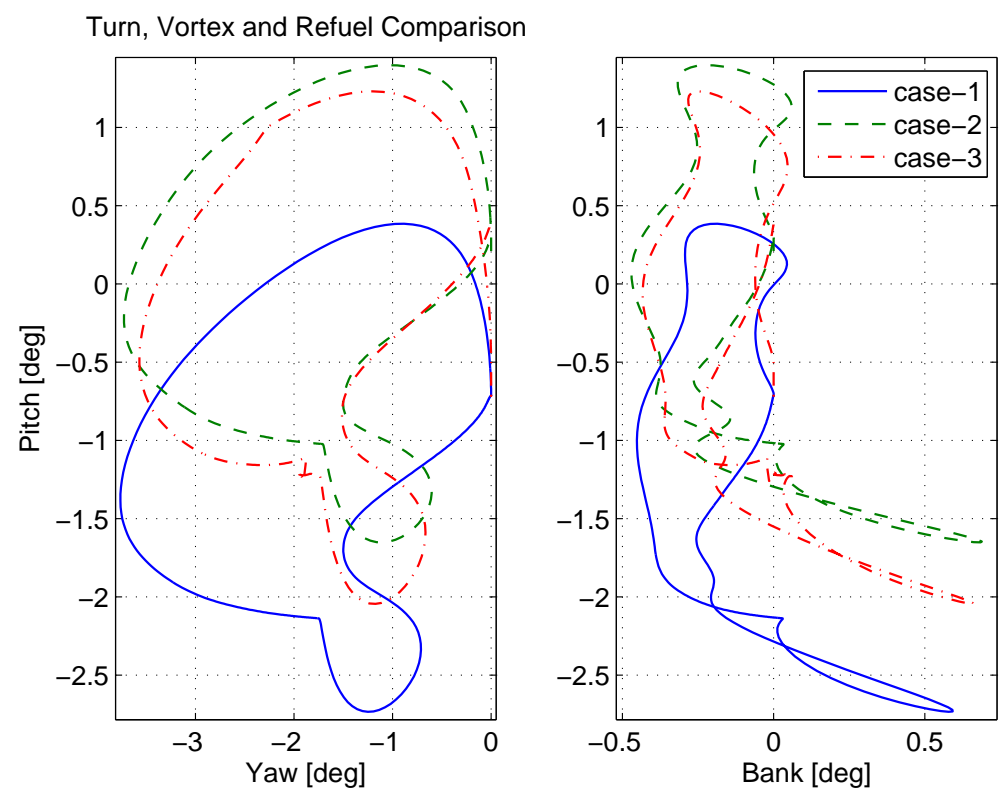

Figure 8.19. Receiver deviation in relative orientation during turn for observation of vortex and refueling effects.

For the purposes of comparison, Fig. 8.17 depicts the receiver behavior in a straight level flight scenario with refueling beginning at 25 seconds as before, but the tanks are now commanded to fill to their full capacities instead of only halfway as previously done. This simulation case is presented to show the response of the receiver in the case of the full-capacity normal refueling scenario. It can be seen that all deviations and control variable variations are larger in magnitude than in the previous scenario to account for the additional mass being transferred into the aircraft.

Finally, Figs. 8.18 and 8.19 are presented as a means of comparing the effect of the vortex on the receiver position and orientation deviations to the effect of the refueling in the turn scenario. In these figures, Case 1 is a simulation involving neither the vortex nor the refueling, and is included as a reference for the other two cases. In Case 2, the vortex is included without any refueling, while Case 3 includes refueling at 25 seconds as before, but with no vortex. From these figures in which the vortex and refueling 
effects are isolated from one another, it is apparent that the vortex and refueling actually contribute extremely similarly to the receiver deviations. From these results, it can be reasonably concluded that the effect of the refueling is as significant to the performance of the receiver as the effect of the vortex-induced wind field. 


\section{CHAPTER 9 CONCLUSION}

In this thesis, a new set of equations of motion of an aircraft is derived to account for (i) time-varying inertia properties due to fuel transfer, (ii) exposure to wind induced by the wake vortex of a lead aircraft, and (iii) position and orientation relative to an accelerating and rotating reference frame.

A system of receiver aircraft and the fuel that will join the aircraft is defined. The expressions for the time rates of change of linear and angular momenta of this sytem of constant mass are derived by formulating the momenta before and after the fuel joins the receiver and moves into the fuel tanks. This allows the application of Newton's Law, which is valid for a system of constant mass. Since the fuel before joining the receiver aircraft is considered in the formulation of the linear and angular momentum variation, the speed and direction of fuel flow into the receiver, as well as the location of the receptacle in the receiver, are included in the equations of motion. The fuel that is transferred into the receiver is modeled as point masses concentrated at the center of mass of fuel within each fuel tank. This alows the formulation of time-varying fuel quantity and center of mass for each fuel tank individually. Further, it allows the specification of fuel tank location, shape, and size in the equations of motion. Additionally, since the fuel flow rate into each fuel tank is individually accounted for in the equations of motion, asymmetric fuel loading and unstead fuel transfer conditions can easily be simulated.

A geometrically fixed body reference frame is chosen for the receiver to address the

fact that the center of mass of the system may change during the fuel transfer. The origin of this body frame is chosen to be at the center of mass of the receiver before the fuel 
transfer. This enables the mass and inertia matrix of the receiver aircraft without fuel to be identified in the derivation of the equations. Thus, the mass and the inertia matrix of the receiver excluding the transferred fuel can be used directly in the equations. Another advantage of writing the equations with respect to a geometrically fixed frame manifests itself when the aerodynamic varibles such as airspeed angle of attack, side slip angle, and stability derivatives are used. Since such variables and derivatives are determined by the shape of the aircraft rather than its mass properties, the standard definitions can be used directly without any modification or re-interpretation.

To derive the equations of motion that include the dynamic effect of wind exposure, the velocity and acceleration of the receiver are expressed as the sum of the velocity and acceleration of the aircraft relative to the the surrounding air, and the velocity and acceleration of the air relative to the inertial frame. This results in equations of motion with explicit wind terms, which facilitiates the incoporation of the wind effect in the aircraft dynamics and kinematics. It should be noted that this approach uses an assumption of uniform wind and wind gradient acting at the origin of the receiver body frame. An averaging technique, which was developed for close-proximity formation flight, is used to approximate the nonuniform wind field induced by the tanker's wake vortex by uniform wind velocity and wind rotation. This enables easy dynamic modeling of the receiver aircraft flying in the wake of the tanker aircraft.

Another novelty in the derivation of the equations is that they are expressed in terms of the position and orientation of the receiver relative to the tanker, rather than relative to the inertial frame. This offers a very suitable set of equations for the aerial refueling problem since aerial refuling requires control of the receiver relative to the tanker. Further, in the final form of the equations, the effects of the tanker's motion on the relative motion of the receiver are clearly shown. This also allows the translational 
and rotational states of the tanker aircraft to be passed into the receiver dynamics as exogenous inputs.

The equations of motion are first derived in vector form. Then, vectrix formalism and rotation matrices are used to convert the equations into state-space matrix form. The matrix form enables the equations of motion to be dirctly implemented in numerical simulation tools such as MATLAB/Simulink. Further, state-space form is preferred for model based control design. Another advantage of the matrix form is that the couplings between rotational and translational dynamics can be easily identified through the appearance of the rotational variables in the translational equations and vice versa.

Application of the derived equations in aerial refueling simulation has demonstrated the ease of implementation and the versatility of the equations in terms of analyzing various types of flight conditions and refueling scenarios. It should be noted that the generic nature of the equations enables the simulation of any type of aircraft at any flight condition with mass transfer of any sort. Analysis of the simulation results has also shown the level and detail of insight that can be gained by the implementation of the equations. In future work, the equations will be utilized in more effective control system development as well as analysis of other systems having time-varying mass properties. 
APPENDIX A

\section{ROTATIONAL KINEMATICS DERIVATION}


Rotational kinematics of the receiver relative to the inertial frame in the form of Poisson's equation is

$$
\mathbf{R}_{\mathbf{B}_{\mathbf{R}} \mathbf{I}} \dot{\mathbf{R}}_{\mathbf{B}_{\mathbf{R}} \mathbf{I}}^{\mathbf{T}}=-\mathbf{S}\left(\omega_{\mathbf{B}_{\mathbf{R}}}\right)
$$

where $\omega_{B_{R}}$ is the representation of the angular velocity of $B_{R^{-}}$frame with respect to the inertial frame and most conveniently defined in $B_{R}$-frame. Similarly for the rotational kinematics of the tanker,

$$
\mathbf{R}_{\mathbf{B}_{\mathbf{T}} \mathbf{I}} \dot{\mathbf{R}}_{\mathbf{B}_{\mathbf{T}} \mathbf{I}}^{\mathbf{T}}=-\mathbf{S}\left(\omega_{\mathbf{B}_{\mathbf{T}}}\right)
$$

where $\omega_{B_{T}}$ is the representation in $B_{T}$-frame of the angular velocity of $B_{T}$-frame.

It is obvious that the angular velocity of the receiver with respect to the inertial frame can be written as the sum of its angular velocity with respect to the tanker and the tanker's angular velocity with respect to the inertial frame. Thus,

$$
\underline{\omega}_{B_{R}}=\underline{\omega}_{B_{R} B_{T}}+\underline{\omega}_{B_{T}}
$$

where $\underline{\omega}_{B_{R}}$ is the angular velocity vector of the receiver relative to the inertial frame, $\underline{\omega}_{B_{R} B_{T}}$ is the angular velocity vector of the receiver relative to the tanker, and $\underline{\omega}_{B_{T}}$ is the angular velocity vector of the tanker relative to the inertial frame. Eq. (A.3) implies

$$
\omega_{B_{R} B_{T}}=\omega_{B_{R}}-\mathbf{R}_{\mathbf{B}_{\mathbf{R}} \mathbf{B}_{\mathbf{T}}} \omega_{B_{T}}
$$

where $\omega_{B_{R} B_{T}}$ and $\omega_{B_{R}}$ are the representations of the respective vectors in $B_{R^{-}}$frame while $\omega_{B_{T}}$ is the representation of the corresponding vector in $B_{T}$-frame, and recall Eq. (2.7) as

$$
\mathbf{R}_{\mathrm{B}_{\mathrm{R}} \mathrm{B}_{\mathrm{T}}}=\mathbf{R}_{\mathrm{B}_{\mathrm{R}} \mathrm{I}} \mathbf{R}_{\mathrm{B}_{\mathrm{T}} \mathbf{I}}^{\mathrm{T}}
$$

Differentiating and transposing Eq. (2.7), we obtain

$$
\dot{\mathbf{R}}_{\mathrm{B}_{\mathrm{R}} \mathrm{B}_{\mathrm{T}}}^{\mathrm{T}}=\mathbf{R}_{\mathrm{B}_{\mathrm{T}} \mathrm{I}} \dot{\mathbf{R}}_{\mathrm{B}_{\mathrm{R}}^{\mathrm{I}}}^{\mathrm{T}}+\dot{\mathbf{R}}_{\mathrm{B}_{\mathrm{T}} \mathrm{I}} \mathbf{R}_{\mathrm{B}_{\mathrm{R}} \mathrm{I}}^{\mathrm{T}}
$$


From Eq. (2.7) and Eq. (A.5), we have

$$
\begin{aligned}
& \mathbf{R}_{\mathrm{B}_{\mathrm{R}} \mathrm{B}_{\mathrm{T}}} \dot{\mathbf{R}}_{\mathrm{B}_{\mathrm{R}}^{\mathrm{T}} \mathrm{B}_{\mathrm{T}}}^{\mathrm{T}}=\mathbf{R}_{\mathrm{B}_{\mathrm{R}} \mathrm{I}} \mathbf{R}_{\mathrm{B}_{\mathrm{T}} \mathrm{I}}^{\mathrm{T}}\left(\mathbf{R}_{\mathrm{B}_{\mathrm{T}} \mathrm{I}} \dot{\mathbf{R}}_{\mathrm{B}_{\mathrm{R}}^{\mathrm{I}}}^{\mathrm{T}}+\dot{\mathbf{R}}_{\mathrm{B}_{\mathrm{T}} \mathrm{I}} \mathbf{R}_{\mathrm{B}_{\mathrm{R}} \mathrm{I}}^{\mathrm{T}}\right) \\
& =\mathbf{R}_{\mathrm{B}_{\mathrm{R}} \mathrm{I}} \dot{\mathbf{R}}_{\mathrm{B}_{\mathrm{R}} \mathrm{I}}^{\mathrm{T}}+\mathbf{R}_{\mathrm{B}_{\mathrm{R}} \mathrm{I}} \mathbf{R}_{\mathrm{B}_{\mathrm{T}} \mathrm{I}}^{\mathrm{T}} \dot{\mathbf{R}}_{\mathrm{B}_{\mathrm{T}} \mathrm{I}} \mathbf{R}_{\mathrm{B}_{\mathrm{R}} \mathrm{I}}^{\mathrm{T}}
\end{aligned}
$$

In the above equation, the first term on the right hand side can be replaced by $-\mathbf{S}\left(\omega_{\mathbf{B}_{\mathbf{R}}}\right)$ owing to Eq. (A.1). The second term can be simplified as shown below. The time derivative of $\mathbf{R}_{\mathbf{B}_{\mathbf{T}} \mathbf{T}}^{\mathbf{T}} \mathbf{R}_{\mathbf{B}_{\mathbf{T}} \mathbf{I}}=\mathbf{I}$, where $\mathbf{I}$ is a $(3 \times 3)$ identity matrix, implies

$$
\mathbf{R}_{\mathbf{B}_{\mathrm{T}} \mathbf{I}}^{\mathrm{T}} \dot{\mathbf{R}}_{\mathrm{B}_{\mathrm{T}} \mathbf{I}}=-\dot{\mathbf{R}}_{\mathrm{B}_{\mathrm{T}} \mathbf{T}}^{\mathbf{T}} \mathbf{R}_{\mathrm{B}_{\mathrm{T}} \mathrm{I}}
$$

which is used for the second term in Eq. (A.6) to get

$$
\begin{aligned}
& \mathbf{R}_{\mathrm{B}_{\mathrm{R}} \mathrm{I}} \mathbf{R}_{\mathrm{B}_{\mathrm{T}} \mathrm{I}}^{\mathrm{T}} \dot{\mathrm{R}}_{\mathrm{B}_{\mathrm{T}} \mathrm{I}} \mathbf{R}_{\mathrm{B}_{\mathrm{R}} \mathrm{I}}^{\mathrm{T}}=-\mathbf{R}_{\mathrm{B}_{\mathrm{R}} \mathrm{I}} \dot{\mathbf{R}}_{\mathrm{B}_{\mathrm{T}} \mathrm{I}}^{\mathrm{T}} \mathbf{R}_{\mathrm{B}_{\mathrm{T}} \mathrm{I}} \mathbf{R}_{\mathrm{B}_{\mathrm{R}} \mathrm{I}}^{\mathrm{T}} \\
& =-\mathbf{R}_{\mathbf{B}_{\mathbf{R}} \mathbf{I}} \mathbf{R}_{\mathbf{B}_{\mathrm{T}} \mathbf{I}}^{\mathbf{T}} \mathbf{R}_{\mathrm{B}_{\mathrm{T}} \mathbf{I}} \dot{\mathbf{R}}_{\mathbf{B}_{\mathrm{T}} \mathbf{I}}^{\mathbf{T}} \mathbf{R}_{\mathrm{B}_{\mathrm{T}} \mathbf{I}} \mathbf{R}_{\mathrm{B}_{\mathbf{R}} \mathbf{I}}^{\mathbf{T}}
\end{aligned}
$$

where the product $\mathbf{R}_{\mathbf{B}_{\mathbf{T}} \mathbf{I}}^{\mathbf{T}} \mathbf{R}_{\mathbf{B}_{\mathbf{T}} \mathbf{I}}$ (which is equal to identity matrix) has been inserted into the right hand side of Eq. (A.8). Grouping terms using Eqs. (A.2) and (2.7) yields

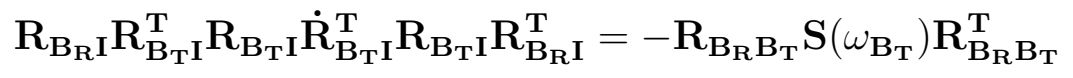

Now, using the property that the cross product is preserved after the transformation by a rotation matrix, it can be shown that

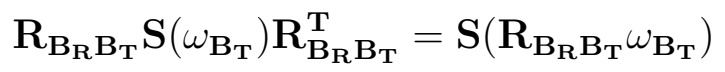

which gives the simplification for the second term on the right hand side of Eq. (A.6) to be

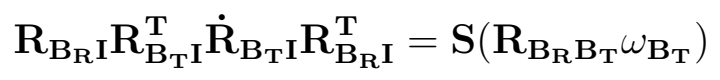


Thus, using Eqs. (A.1), (A.4) and (A.12), along with the property of linearity for $\mathbf{S}(\cdot)$ operations in Eq. (A.6), Eq. (5.6) is proven as

$$
\begin{aligned}
\mathbf{R}_{\mathbf{B}_{\mathbf{R}} \mathbf{B}_{\mathbf{T}}} \dot{\mathbf{R}}_{\mathbf{B}_{\mathbf{R}} \mathbf{B}_{\mathbf{T}}}^{\mathbf{T}} & =-\mathbf{S}\left(\omega_{\mathbf{B}_{\mathbf{R}}}\right)+\mathbf{S}\left(\mathbf{R}_{\left.\mathbf{B}_{\mathbf{R}} \mathbf{B}_{\mathbf{T}} \omega_{\mathbf{B}_{\mathbf{T}}}\right)}\right. \\
& =-\mathbf{S}\left(\omega_{\mathbf{B}_{\mathbf{R}}}-\mathbf{R}_{\left.\mathbf{B}_{\mathbf{R}} \mathbf{B}_{\mathbf{T}} \omega_{\mathbf{B}_{\mathbf{T}}}\right)}\right. \\
& =-\mathbf{S}\left(\omega_{\mathbf{B}_{\mathbf{R}} \mathbf{B}_{\mathbf{T}}}\right)
\end{aligned}
$$

By rearranging Eq. (A.4), it is shown that $\omega_{B_{R}}=\omega_{B_{R} B_{T}}+\mathbf{R}_{\mathbf{B}_{\mathbf{R}} \mathbf{B}_{\mathbf{T}}} \omega_{B_{T}}$. Taking the derivative of this expression yields

$$
\dot{\omega}_{B_{R}}=\dot{\omega}_{B_{R} B_{T}}+\dot{\mathbf{R}}_{\mathbf{B}_{\mathbf{R}} \mathbf{B}_{\mathbf{T}}} \omega_{B_{T}}+\mathbf{R}_{\mathbf{B}_{\mathbf{R}} \mathbf{B}_{\mathbf{T}}} \dot{\omega}_{B_{T}}
$$

If Eq. (A.13) is rearranged such that $\dot{\mathbf{R}}_{\mathbf{B}_{\mathbf{R}} \mathbf{B}_{\mathbf{T}}}^{\mathbf{T}}=-\mathbf{R}_{\mathbf{B}_{\mathbf{R}} \mathbf{B}_{\mathbf{T}}}^{\mathbf{T}} \mathbf{S}\left(\omega_{\mathbf{B}_{\mathbf{R}} \mathbf{B}_{\mathbf{T}}}\right)$, then it follows that

$$
\dot{\mathbf{R}}_{\mathrm{B}_{\mathbf{R}} \mathbf{B}_{\mathrm{T}}}=\mathbf{S}\left(\omega_{\mathrm{B}_{\mathbf{R}} \mathbf{B}_{\mathrm{T}}}\right) \mathbf{R}_{\mathrm{B}_{\mathbf{R}} \mathbf{B}_{\mathrm{T}}}
$$

By substituting Eq. (A.15) into Eq. (A.14), an expression for $\dot{\omega}_{B_{R}}$ is arrived at as

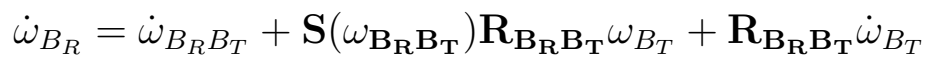

thus completing the proof of Eq. (5.9). 
APPENDIX B

DERIVATIONS OF POSITION VECTORS IN MATRIX FORM 
Recall from Eq. (5.21) that

$$
\underline{\underline{\rho}}_{m_{j}}=\left[\underline{\rho}_{m_{j}}\right]_{B_{R}}+\underline{\omega}_{B_{R}} \times \underline{\rho}_{m_{j}}
$$

Substituting $\underline{\omega}_{B_{R}}$ from Eq. (5.7) gives

$$
\underline{\dot{\rho}}_{m_{j}}=\left[\underline{\dot{\rho}}_{m_{j}}\right]_{B_{R}}+\underline{\omega}_{B_{R} B_{T}} \times \underline{\rho}_{m_{j}}+\underline{\omega}_{B_{T}} \times \rho_{m_{j}}
$$

Recall that $\underline{\rho}_{m_{j}}=\left[\underline{\hat{B}}_{R}\right]^{T} \rho_{m_{j}}, \underline{\omega}_{B_{R} B_{T}}=\left[\underline{\hat{B}}_{R}\right]^{T} \omega_{B_{R} B_{T}}, \underline{\omega}_{B_{T}}=\left[\underline{\hat{B}}_{T}\right]^{T} \omega_{B_{T}}$, and $\left[\underline{\hat{B}}_{R}\right]=$ $\mathbf{R}_{\mathbf{B}_{\mathbf{R}} \mathbf{B}_{\mathbf{T}}}\left[\underline{B}_{T}\right]$. Thus, Eq. (B.1) becomes

$$
\underline{\dot{\rho}}_{m_{j}}=\left[\underline{\hat{B}}_{R}\right]^{T} \dot{\rho}_{m_{j}}+\left[\underline{\hat{B}}_{R}\right]^{T} \omega_{B_{R} B_{T}} \times\left[\underline{\hat{B}}_{R}\right]^{T} \rho_{m_{j}}+\left[\underline{\hat{B}}_{R}\right]^{T} \mathbf{R}_{\mathbf{B}_{\mathbf{R}} \mathbf{B}_{\mathbf{T}}} \omega_{B_{T}} \times\left[\underline{\hat{B}}_{R}\right]^{T} \rho_{m_{j}}
$$

Using the vector product property in Eq. (2.15) yields

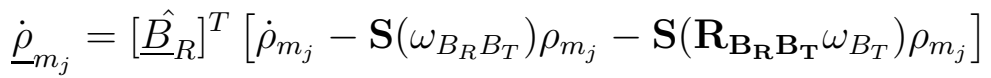

Note that $\underline{\dot{\rho}}_{m_{j}}$ can be expressed in the inertial frame using $\left[\underline{\hat{B}}_{R}\right]^{T}=[\underline{\hat{I}}]^{T} \mathbf{R}_{\mathbf{B}_{\mathbf{T}} \mathbf{I}}^{\mathbf{T}} \mathbf{R}_{\mathbf{B}_{\mathbf{R}} \mathbf{B}_{\mathbf{T}}}^{\mathbf{T}}$, which yields Eq. (5.22).

Recall Eq. (5.23) as being

$$
\underline{\ddot{\rho}}_{m_{j}}=\left[\underline{\ddot{\rho}}_{m_{j}}\right]_{B_{R}}+2 \underline{\omega}_{B_{R}} \times\left[\underline{\dot{\rho}}_{m_{j}}\right]_{B_{R}}+\underline{\dot{\omega}}_{B_{R}} \times \underline{\rho}_{m_{j}}+\underline{\omega}_{B_{R}} \times\left(\underline{\omega}_{B_{R}} \times \underline{\rho}_{m_{j}}\right)
$$

After substituting $\underline{\omega}_{B_{R}}$ from Eq. (5.7) and rearranging, the above equation becomes

$$
\begin{aligned}
\underline{\ddot{\rho}}_{m_{j}} & =\left[\underline{\ddot{\rho}}_{m_{j}}\right]_{B_{R}}+2 \underline{\omega}_{B_{R} B_{T}} \times\left[\underline{\dot{\rho}}_{m_{j}}\right]_{B_{R}}+2 \underline{\omega}_{B_{T}} \times\left[\underline{\dot{\rho}}_{m_{j}}\right]_{B_{R}}+\underline{\dot{\omega}}_{B_{R} B_{T}} \times \underline{\rho}_{m_{j}} \\
& +\underline{\dot{\omega}}_{B_{T}} \times \underline{\rho}_{m_{j}}+\underline{\omega}_{B_{R} B_{T}} \times\left(\underline{\omega}_{B_{R} B_{T}} \times \underline{\rho}_{m_{j}}\right)+\underline{\omega}_{B_{R} B_{T}} \times\left(\underline{\omega}_{B_{T}} \times \underline{\rho}_{m_{j}}\right) \\
& +\underline{\omega}_{B_{T}} \times\left(\underline{\omega}_{B_{R} B_{T}} \times \underline{\rho}_{m_{j}}\right)+\underline{\omega}_{B_{T}} \times\left(\underline{\omega}_{B_{T}} \times \underline{\rho}_{m_{j}}\right)
\end{aligned}
$$

where note that $\underline{\dot{\omega}}_{B_{R} B_{T}}=\left[\underline{\dot{\omega}}_{B_{R} B_{T}}\right]_{B_{R}}+\underline{\omega}_{B_{T}} \times \underline{\omega}_{B_{R} B_{T}}$. After $\underline{\dot{\omega}}_{B_{R} B_{T}}$ is substituted in and the identity of Eq. (2.11) is used, it can be shown that

$\left(\underline{\omega}_{B_{T}} \times \underline{\omega}_{B_{R} B_{T}}\right) \times \underline{\rho}_{m_{j}}+\underline{\omega}_{B_{R} B_{T}} \times\left(\underline{\omega}_{B_{T}} \times \underline{\rho}_{m_{j}}\right)+\underline{\omega}_{B_{T}} \times\left(\underline{\omega}_{B_{R} B_{T}} \times \underline{\rho}_{m_{j}}\right)=2 \underline{\omega}_{B_{T}} \times\left(\underline{\omega}_{B_{R} B_{T}} \times \underline{\rho}_{m_{j}}\right)$ 
Substituting this back into Eq. (B.4) in place of the three terms, using the vector product property of Eq. (2.15), and rearranging, Eq. (B.4) becomes

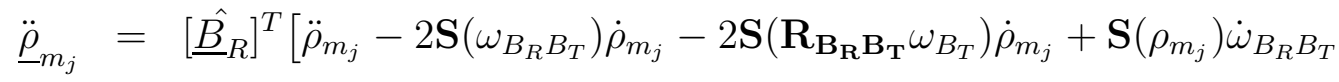

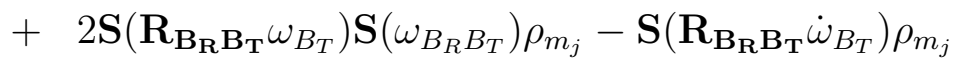

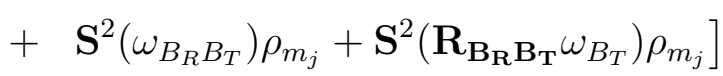

Note that in rearranging Eq. (B.4) to get Eq. (B.6), the skew-symmetric matrix properties of Eqs. (2.22) and (2.17) are used. Similarly to $\underline{\underline{\rho}}_{m_{j}}$, Eq. (5.24) is obtained once $\ddot{\underline{\rho}}_{m_{j}}$ in Eq. (B.6) is expressed in the inertial frame. 


\section{REFERENCES}

[1] M. A. Bennington and K. D. Visser, "Aerial refueling implications for commercial aviation," AIAA Journal of Aircraft, vol. 42:2, pp. 366-375, 2005.

[2] A. Bloy and V. Trochalidis, "Performance and longitudinal stability and control of large receiver aircraft during air to air refueling," in Aeronautical Journal, vol. 93:930, 1989, pp. 367-378.

[3] A. Bloy, M. West, K. Lea, and M. Jouma, "Lateral aerodynamics interference between tanker and receiver in air-to-air refueling," in Journal of Aircraft, vol. 30:5, 1993, pp. 705-710.

[4] A. Bloy and M. West, "Interference between tanker wing wake with roll-up and receiver aircraft," in Journal of Aircraft, vol. 31:5, 1994, pp. 1214-1216.

[5] A. Bloy and M. Jouma'a, "Lateral and directional stability and control in air-toair refueling," in Proceedings of the Institution of Mechanical Engineers, Part G: Journal of Aerospace Engineering, vol. 209:4, 1995, pp. 299-305.

[6] A. Bloy and K. Lea, "Directional stability of a large receiver aircraft in air-to-air refueling," in Journal of Aircraft, vol. 32:2, 1995, pp. 453-455.

[7] M. Pachter, C. Houpis, and D. Trosen, "Design of an air-to-air automatic refueling flight control system using quantitative feedback theory," in International Journal of Robust and Nonlinear Control, vol. 7:6, 1997, pp. 561-580.

[8] J. Dargan, M. Pachter, and J. D'Azzo, "Automatic formation flight control," in Proceedings of the 1992 AIAA GNC Conference, 1992, pp. 838-57. 
[9] W. Blake and D. Multhopp, "Design, performance and modeling considerations for close formation flight," in Proceedings of the 1998 AIAA GNC Conference, Boston, MA, Jul. 1998, pp. 476-86, paper no. 4343.

[10] J. Boskovic, S.-M. Li, and R. Mehra, "Semi-globally stable formation flight control design in three dimensions," in Proceedings of 40th Conference on Decision and Control, 2001, pp. 1059-1064.

[11] P. Binetti, K. Ariyur, M. Krstic, and F. Bernelli, "Control of formation flight via extremum seeking," in Proceedings of the American Control Conference, vol. 4, 2002, pp. 2848-2853.

[12] J. Boskovic, S.-M. Li, and R. Mehra, "Formation flight control design in the presence of unknown leader commands," in Proceedings of the American Control Conference, vol. 4, 2002, pp. 2854-2859.

[13] S. Singh, P. Chandler, C. Schumacher, S. Banda, and M. Pachter, "Nonlinear adaptive close formation control of unmanned aerial vehicles," Dynamics and Control, vol. 10:2, pp. 179-194, 2000.

[14] M. Innocenti, G. Mancino, and M. Garofoli, "Preliminary analysis of formation flight management," in Proceedings of AIAA Guidance, Navigation, and Control Conference and Exhibit, Portland, OR, Aug. 9-11, 1999, aIAA-1999-3984.

[15] C. Schumacher and R. Kumar, "Adaptive control of uavs in close-coupled formation flight," in Proceedings of the American Control Conference, vol. 2, 2000, pp. 849-853.

[16] C. Schumacher and S. N. Singh, "Nonlinear control of multiple uavs in close-coupled formation flight," in Proceedings of AIAA Guidance, Navigation, and Control Conference and Exhibit, Denver, CO, Aug. 14-17, 2000, aIAA-2000-4373.

[17] D. Chichka, J. Speyer, and C. Park, "Peak-seeking control with application to formation flight," in Proceedings of the 38th IEEE Conference on Decision $\&$ Control, vol. 3, 1999, pp. 2463-2470. 
[18] R. Fierro, C. Belta, J. Desai, and V. Kumar, "On controlling aircraft formations," in Proceedings of the 40th IEEE Conference on Decision \& Control, vol. 2, 2001, pp. 1065-1070.

[19] Y. Pennecot, E. M. Atkins, and R. M. Sanner, "Intelligent spacecraft formation management and path planning," in Proceedings of AIAA Aerospace Sciences Meeting and Exhibit, Reno, NV, Jan. 14-17, 2002, aIAA-2002-1072.

[20] S. Welsh and K. Subbarao, "Adaptive synchronization and control of free flying robots for capture of dynamic free-floating spacecrafts," in Proceedings of AIAA/AAS Astrodynamics Specialist Conference and Exhibit, Providence, RI, Aug. 16-19, 2004, aIAA-2004-5298.

[21] Y. Tsuda and N. Shinichi, "New attitude motion following control algorithm for capturing tumbling object in space," Acta Astronautica, vol. 53, pp. 847-861, 2003.

[22] K. Isao, M. Mokuno, K. Toru, and T. Suziki, "Result of automous rendezvous docking experiment of engineering test satellite-vii," AIAA Journal of Spacecraft and Rockets, vol. 38:1, pp. 105-111, Jan-Feb 2001.

[23] P. Singla, K. Subbarao, and J. L. Junkins, "Adaptive output feedback control for spacecraft rendezvous and docking under measurement uncertainty," AIAA Journal of Guidance, Control, and Dynamics, vol. 29:4, pp. 892-902, 2006.

[24] C.-F. Lin, J. Bibel, N. Hecht, and D. Serakos, "Autonomous integrated air vehicle control," in Proceedings of AIAA and SAE World Aviation Conference, Anaheim, CA, Sept. 28-30, 1998, aIAA-1998-5518.

[25] R. Hess, "A simplified technique for modeling piloted rotorcraft operations near ships," in Proceedings of AIAA Atmospheric Flight Mechanics Conference and Exhibit, San Francisco, CA, Aug. 15-18, 2005, aIAA-2005-6030. 
[26] D. Lee, J. Horn, N. Sezer-Uzol, and L. Long, "Simulation of pilot control activity during helicopter shipboard operations," in Proceedings of AIAA Atmospheric Flight Mechanics Conference and Exhibit, Austin, TX, Aug. 11-14, 2003, aIAA-2003-5306.

[27] A. Dogan, S. Sato, and W. Blake, "Flight control and simulation for aerial refueling," in Proceedings of AIAA Guidance, Navigation, and Control Conference, San Francisco, CA, Aug 2005, aIAA paper 2005-6264.

[28] E. Kim, A. Dogan, and W. Blake, "Control of a receiver aircraft relative to the tanker in racetrack maneuver," in Proceedings of the 2006 AIAA GNC Conference, Keystone, CO, 2006, paper no. 6710.

[29] J. B. Rosser, R. R. Newton, and G. L. Gross, Mathematical Theory of Rocket Flight. McGraw-Hill Book Company, Inc., 1947.

[30] K. Jarmolow, "Dynamics of a spinning rocket with varying inertia and applied moment," Journal of Applied Physics, vol. 28:3, pp. 308-313, 1957.

[31] J. W. Ellis and C. W. McArthur, "Applicability of euler's dynamical equations to rocket motion," ARS Journal, vol. 29:11, pp. 863-864, 1959.

[32] F. O. Eke, "Dynamics of variable mass systems with application to the star 48 solid rocket motor," Advances in Astronautical Sciences, vol. 54:2, pp. 671-684, 1984.

[33] W. T. Thomson, Introduction to Space Dynamics. Mineola, NY: Dover Publications, Inc., 1986.

[34] F. O. Eke, "Angular momentum and multibody dynamics," Advances in Astronautical Sciences, vol. 75:1, pp. 365-376, 1991.

[35] F. O. Eke and E. Cervantes, "Dynamics of axisymmetric rockets in free flight," Journal of Dynamic Systems, Measurement, and Control, vol. 120:3, pp. 410-414, 1998.

[36] F. O. Eke and T. C. Mao, "On the dynamics of variable mass system," International Journal of Mechanical Engineering Education, vol. 30:2, pp. 123-137, 2002. 
[37] F. O. Eke, T. C. Mao, and M. J. Morris, "Free attitude motions of a spinning body with substantial mass loss," Journal of Applied Mechanics, vol. 71:2, pp. 190-194, 2004.

[38] J. D. Schierman, D. Ward, J. Monaco, J. Hull, and T. Lawrence, "On-line identification and nonlinear control of rotorcraft/external-load systems," in Proceedings of AIAA Guidance, Navigation, and Control Conference and Exhibit, Denver, CO, 14-17 August 2000, aIAA-2000-4042.

[39] D. Collard, "Future supersonic transport studies at aerospatiale," SAE (Society of Automotive Engineers) Transactions, vol. 99, pp. 1700-1708, 1990.

[40] — - "Concorde airframe design and development," SAE (Society of Automotive Engineers) Transactions, vol. 100, pp. 2620-2641, 1991.

[41] C. Orlebar, The Concorde Story. London, United Kingdom: The Hamlyn Publishing Group Limited, 1994.

[42] J. Shen and N. H. McClamroch, "Translational and rotational spacecraft maneuvers via shape change actuators," in Proceedings of the American Control Conference, Arlington, VA, June 25-27, 2001.

[43] C. Woolsey and N. Leonard, "Moving mass control for underwater vehicles," in Proceedings of the American Control Conference, Anchorage, AK, May 8-10 2002.

[44] C. Schultz and C. Woolsey, "An experimental platform for validating internal actuator control strategies," in IFAC Workshop on Guidance and Control of Underwater Vehicles, Newport, South Wales, U.K., April 2003.

[45] B. Coblei, "Capabilities and future applications of the nasa autonomous formation flight (aff) aircraft," in 1st AIAA UAV Conference, Portsmouth, VA, May 2002.

[46] J. Valasek, J. Kimmett, D. Hughes, K. Gunnam, and J. Junkins, "Vision based sensor and navigation system for autonomous aerial refueling," in 1st AIAA UAV Conference, Portsmouth, VA, May 2002. 
[47] K. Johnson and K. Awni, "A roll autopilot for autonomous air refueling," in AIAA Guidance, Navigation, and Control Conference and Exhibit, Monterey, CA, Aug 2002.

[48] M. Fravolini, A. Ficola, M. Napolitano, G. Campa, and M. Perhinschi, "Development of modelling and control tools for aerial refueling for uavs," in AIAA Guidance, Navigation, and Control Conference and Exhibit, Austin, TX, Aug 2003.

[49] G. Campa, B. Seanor, M. Perhinschi, M. Fravolini, A. Ficola, and M. Napolitano, "Autonomous aerial refueling for uavs using a combined gps-machine vision guidance," in AIAA Guidance, Navigation, and Control Conference and Exhibit, Providence, RI, Aug 2004.

[50] V. S. E. Lavretsky and N. Hovakimyan, "Aerial refueling autopilot design methodology: Application to f-16 aircraft model," in AIAA Guidance, Navigation, and Control Conference and Exhibit, Providence, RI, Aug 2004.

[51] J. Hansen, J. Murray, and N. Campos, "The nasa dryden aar project: A flight test approach to an aerial refueling system," in AIAA Atmospheric Flight Mechanics Conference and Exhibit, Providence, RI, Aug 2004.

[52] A. Bloy and M. Khan, "Modeling of the receiver aircraft in air-to-air refueling," in AIAA Journal of Aircraft, vol. 38, no. 2, 2001, pp. 393-96.

[53] S. Venkataramanan and A. Dogan, "Dynamic effects of trailing vortex with turbulence \& time-varying inertia in aerial refueling," in to be presented in the 2004 AIAA AFM Conference, Providence, RI, Aug. 2004.

[54] W. Jewell and R. Stapleford, "Mathematical models used to simulate aircraft encounters with wake vortices," in STI Technical Report 1035-4, 1975, pp. 38-57.

[55] M. Pachter, J. D’Azzo, and A. Proud, "Tight formation flight control," in J. Guidance, Control, and Dynamics, vol. 24:2, 2001, pp. 246-254. 
[56] W. Johnson, G. Teper, and H. Rediess, "Study of control system effectiveness in alleviating vortex wake upsets," J. Aircraft, vol. 11:3, pp. 148-154, 1974.

[57] S. Venkataramanan and A. Dogan, "Nonlinear control for reconfiguration of uav formation," in Proceedings of the 2003 AIAA GNC Conference, Austin, TX, Aug. 2003, paper no. 5725 .

[58] A. Dogan, S. Venkataramanan, and W. Blake, "Modeling of aerodynamic coupling between aircraft in close proximity," AIAA Journal of Aircraft, vol. 42:4, pp. 941955, Jul-Aug 2005.

[59] E. Kim, "Control and simulation of relative motion for aerial refueling in racetrack maneuver," Master's thesis, The University of Texas at Arlington, Arlington, TX, May 2007. 


\section{BIOGRAPHICAL STATEMENT}

JAYME WAISHEK was born in Euless, Texas, and began undergraduate studies at the University of Texas at Arlington in 2002. While an undergraduate, she was employed by Heli-Dyne System, Inc, as an engineering apprentice under the mechanical and electrical engineering staff and was additionally the president of the UTA student chapter of the Sigma Gamma Tau aerospace honorary. She earned an Honors Bachelor of Science degree in aerospace engineering in 2006 and immediately transitioned to the Masters program at UTA. She was simultaneously hired by the Automatric Flight Controls Design group within the Bell Helicopter engineering department, working there the entire duration of her graduate studies. As a graduate student, she was also a Graduate Research Assistant in the Computer Aided Control Systems Design (CACSD) Lab under the supervision of Dr. Atilla Dogan and later worked as his Graduate Teaching Assistant. During this time, she published a paper entitled "Derivation of the Equations of Motion of Receiver Aircraft in Aerial Refueling" in the proceedings of ASM (Aerospace Sciences Meeting) in January, 2007. She received numerous engineering scholarship awards during her undergraduate career and, as a graduate student, she has been a two-time awardee

of the Texas Space Grant Consortium Fellowship program. She is a student member of AIAA and Alpha Chi. 